\title{
Microlocal Analysis and Calculations on Some Relatively Invariant Hyperfunctions Related \\ to Zeta Functions Associated with the Vector Spaces of Quadratic Forms
}

\author{
By \\ Masakazu MURO*
}

\section{Introduction}

In this paper, we shall give explicit formulas of the Fourier transforms of some relatively invariant hyperfunctions on the vector spaces of quadratic forms and some similar Hermitian forms. These calculations are applicable to the calculations of the functional equations and residues of zeta functions associated with each vector spaces. (See Sato-Shintani [5] and Shintani [21]). Sato-Shintani [5] and Shintani [21] has calculated some of them in a classical way, but, in this paper, we shall give more results by making use of a different way.

Let $\mathbb{V}_{\boldsymbol{R}}$ be the real vector space consisting of $n \times n$ symmetric matrices over $\mathbb{R}$. The real linear algebraic group $\mathbb{G}_{R}=\mathbb{G} \mathbb{L}(n, \mathbb{R})^{+}$acts on this vector space $\mathbb{V}_{R}$ by

$$
(g, x) \longmapsto g \circ x \circ^{t} g, \quad\left(g \in \mathbb{G}_{R} \text { and } x \in \mathbb{V}_{R}\right),
$$

and it is a rational representation of $\mathbb{G}_{R}$ on $\mathbb{V}_{R}$. Then $\mathbb{V}_{R}$ decomposes into a finite number of orbits, and each orbit is parametrized by its signature. We denote by $\boldsymbol{S}_{i}^{j}$ the orbit consisting of the points whose signature is $(n-i-j, j)$. If $i=0$, then $\mathbb{S}_{0}^{j}$ is an open orbit in $V_{R}$ and there exists a unique relatively invariant hyperfunction corresponding to the character $\chi(g)^{s}=\operatorname{det}(g)^{2 s}(s \in \mathbb{C})$ which depends on $s$ meromorphically and is supported on $\boldsymbol{S}_{0}^{j}$. The calculations of the Fourier transforms of such relatively invariant hyperfunctions with a meromorphic

Communicated by M. Kashiwara, October 23, 1984. Revised November 25, 1985.

*Department of Mathematics, Kochi University, Kochi 780, Japan. 
parameter $s \in \boldsymbol{C}$ is equivalent to the computations of the functional equations of the zeta functions associated with quadratic forms which is denoted by $\xi_{i}^{(n)}(s, L)$ in Shintani [21]. If $i \geqq 1$, then $\boldsymbol{S}_{i}^{j}$ is a locally closed orbit and its dimension is less than the dimension of $V_{R}$. We shall show that there exists a unique $\boldsymbol{S L}(n, \boldsymbol{R})$-invariant measure on each $\boldsymbol{S}_{i}^{j}$ and that it is extended as a tempered distribution on $\boldsymbol{V}_{\boldsymbol{R}}$ supported on $\overline{\boldsymbol{S}}_{i}^{j}$ and compute the Fourier transform of it. By using the Poisson's summation formula, we can compute some contributions to the residues of $\xi_{k}^{(n)}(s, L)$ in terms of the explicit computations of the Fourier transforms. But, so far, we can not compute all the contributions by our results. For details, see Sato-Shintani [5] and Shintani [21]. Similarly, for the vector space $V_{\boldsymbol{R}}$ of complex Hermitian forms, we shall compute the same things. In such cases, we can compute the residues of the zeta functions associated with them completely from our calculations by making use of the method of Sato-Shintani [5].

In this paper, we start with the applications of the arguments in Sato-Kashiwara-Kimura-Oshima [6], which we abbreviate S-K-K-O, to the cases we shall deal with in this paper (\$1). S-K-K-O [6] gave a method to examine a holonomic system of relatively invariant hyperfunctions. In $\S 2$, we restrict the holonomic systems to the real form $V_{R}$ and investigate the real structures of them. The main result of this section is an application of Kashiwara's theorem to our cases (Proposition 2.11). Kashiwara's theorem (Theorem 2.8) was proved in Kashiwara and Miwa [8]. In Chapter II, we regard relatively invariant hyperfunctions as solutions of the holonomic systems which we shall investigate in Chapter I. We shall compute the Fourier transforms of $|\operatorname{det} x|^{s}$ (Theorem 3.6) and construct the hyperfunctions $T_{i}^{j}(x)$ whose support is contained in a closure of $\boldsymbol{G}_{R}$-orbit $\boldsymbol{S}_{i}^{j}$ and which is a relatively invariant measure on $\boldsymbol{S}_{i}^{j}$ (Theorem 4.1). Moreover, we compute the Fourier transform of $T_{i}^{j}(x)$ (Theorem 4.3). Among the above results, the Fourier transforms of $|\operatorname{det} x|^{s}$ have been computed by some authors. Above all, T. Suzuki [37] has computed them by utilizing microlocal analysis.

The main results of this paper are concentrated in $\S 4$. In almost all parts of the explanations on microlocal analysis are due to M. Kashiwara (Kashiwara-Miwa [8]) but the author added some complementaries for our computations. 
The author would like to express his hearty thanks to Professor M. Sato and Professor M. Kashiwara for their encouragement. The author would also like to thanks to Professor T. Kimura, F. Sato and T. Oshima for their helpful advice.

\section{Contents}

Chapter I. Holonomic Systems.

$\S 1$ Holonomic systems of relative invariants (Reviews from the $\mathrm{S}-\mathrm{K}-\mathrm{K}-\mathrm{O}$ [6] theory).

$\S 2$ Real forms of holonomic systems and real principal symbols, (Applications of Kashiwara's result).

Chapter II. Constructions of relatively invariant hyperfunctions and the Fourier transforms.

$\S 3$ The hyperfunctions $|P|_{i}^{s}(x)$ and their Fourier transforms.

$\S 4$ Invariant measures on singular orbits and their Fourier transforms.

\section{Notations}

We denote by $\mathbb{Z}, \mathbb{Q}, \mathbb{R}$ and $\mathbb{C}$, the ring of rational integers, the rational number field, the real number field and the complex number field, respectively. For any ring $\mathbb{F}$, we denote $\mathbb{R}(n, m, \mathbb{F}$ ) (resp. $\mathbb{M}(n, \mathbb{F})$ ) the set of $n \times m$ (resp. $n \times n)$ matrices. We denote by $G \mathbb{L}(n, F)$ the group of invertible elements in $\mathbb{M}(n, \mathbb{F})$. For any finite dimensional real vector space $\boldsymbol{V}, \mathscr{S}(\mathbb{V})$ is the space of rapidly decreasing functions on $\mathbb{V}$.

\section{Chapter I. Holonomic Systems}

In this chapter, we shall examine the microlocal structures of relatively invariant hyperfunctions by analyzing the holonomic systems and holonomy diagrams.

\section{$\S 1$. Holonomic Systems of Relative Invariants, (Reviews from the S-KM-K-O [6] theory)}

The contents of this section is essentially due to $\mathrm{S}-\mathrm{K}-\mathrm{K}-\mathrm{O}$ [6] and T. Kimura [24]. For the details, see S-K-K-O [6] and T. Kimura [24]. 
Let $\left(\boldsymbol{G}_{\boldsymbol{C}}, \rho, \boldsymbol{V}_{\boldsymbol{C}}\right)$ be a regular prehomogeneous vector space, which is one of the followings.

1) $\boldsymbol{G}_{C}=\boldsymbol{G L}(n, \boldsymbol{C})$,

$$
\boldsymbol{V}_{\boldsymbol{C}}=\boldsymbol{S y \boldsymbol { m }}(n, \boldsymbol{C}):=\left\{x \in \boldsymbol{M}(n, \boldsymbol{C}) ;{ }^{t} x=x\right\},
$$

$\rho(g) ; x \longmapsto g \cdot x \cdot{ }^{t} g,\left(g \in \boldsymbol{G}_{C}, x \in V_{C}\right)$.

2) $\boldsymbol{G}_{\boldsymbol{C}}=\boldsymbol{G L}(n, \boldsymbol{C}) \times \boldsymbol{G} \boldsymbol{L}(n, \boldsymbol{C})$,

$\boldsymbol{V}_{\boldsymbol{c}}=\boldsymbol{M}(n, \boldsymbol{C})$,

$\rho(g) ; x \longmapsto g_{1} \cdot x \cdot{ }^{t} g_{2}, \quad\left(g=\left(g_{1}, g_{2}\right) \in G_{C}, x \in V_{C}\right)$.

3) $\boldsymbol{G}_{\boldsymbol{C}}=\boldsymbol{G} \boldsymbol{L}(2 n, \boldsymbol{C})$,

$\boldsymbol{V}_{\boldsymbol{C}}=\boldsymbol{A l t}(2 n, \boldsymbol{C}) ;=\left\{x \in \boldsymbol{M}(2 n, \boldsymbol{C}) ;{ }^{t} x=-x\right\}$,

$\rho(g) ; x \longmapsto g \cdot x \bullet^{t} g,\left(g \in G_{C}, x \in V_{C}\right)$.

Irreducible relative invariants $P(x)$ are by definition

1) $P(x)=(\operatorname{det} x)$,

2) $P(x)=(\operatorname{det} x)$,

3) $P(x)=($ Pff $x)$,

respectively. Here ( $\operatorname{det} x$ ) means the determinant of a matrix $x$ and (Pff $x$ ) means the Pffafian of an alternative matrix $x$. The corresponding characters $\chi$, i. e., $P(\rho(g) x)=\chi(g) P(x)$, are

1) $\chi(g)=(\operatorname{det} g)^{2}$,

2) $\chi(g)=\left(\operatorname{det} g_{1}\right)\left(\operatorname{det} g_{2}\right)$,

3) $\chi(g)=\operatorname{det} g$,

respectively. The inner products $<,>$ on $V_{c}$ are defined by

$$
\langle x, y\rangle=\operatorname{tr}\left(x^{t} y\right),
$$

and we identify $\boldsymbol{V}_{\boldsymbol{C}}$ and its dual space $\boldsymbol{V}_{\boldsymbol{c}}^{*}$ by (1.3). The contragredient representation $\rho^{*}$ of $\rho$ defines a prehomogeneous vector space having the same relative invariant $P(x)$, whose corresponding character is $\chi^{-1}(g)$.

Let $\mathscr{G}_{c}$ be the Lie algebra of $\boldsymbol{G}_{\boldsymbol{C}}, d \rho$ the infinitesimal representation of $\rho$ and $\delta \chi$ the infinitesimal character of $\chi_{0}$. Then we have the equation,

$$
\left(<d \rho(A) x, D_{x}>-s \delta \chi(A)\right) P^{s}(x)=0\left(A \in \mathscr{G}_{c}\right) .
$$

Here $P^{s}(x)$ means a generator of $\mathscr{D}_{V_{C}}$-Module, where $\mathscr{D}_{V_{C}}$ is the sheaf of differential operators on $V_{c}$, not a function. We consider the system 
of differential equations,

$$
\mathfrak{M}_{s} ;\left(<d \rho(A) x, D_{x}>-s \delta \chi(A)\right) u=0 \quad\left(A \in \mathscr{G}_{c}\right) .
$$

We shall examine the system of differential equation (1.4) following to the arguments in $\mathrm{S}-\mathrm{K}-\mathrm{K}-\mathrm{O}[6]$.

The characteristic variety of $\mathfrak{M}_{s}$ is by definition,

$$
\left\{(x, y) \in \mathbb{T}^{*} \mathbb{V}_{c} ;\langle d \rho(A) x, y\rangle=0, A \in \mathscr{G}_{c}\right\}
$$

and we denote it by $\operatorname{ch}\left(\mathfrak{M}_{s}\right)$ or for simplicity, by $\mathfrak{C}^{5}$ We identify $T^{*} V_{c}^{*}$ and $V_{C} \times V_{C}^{*}$. Then the group $G_{c}$ acts on $\mathbb{T}^{*} \mathbb{V}_{C}$ naturally. The characteristic variety $\mathbb{S}$ is an invariant subvariety under the actions of $G_{c}$ because

$$
\left\langle d \rho(A) \rho(g) x, \rho^{*}(g) y>=\left\langle\rho(g)^{-1} d \rho(A) \rho(g) x, y\right\rangle,\right.
$$

and

$$
d \rho(A) \longmapsto \rho(g)^{-1} d \rho(A) \rho(g)
$$

defines an automorphism of $d \rho\left(\mathscr{G}_{c}\right)$. Therefore, the sets $\left\{(x, y) \in \mathbb{T}^{*} \mathbb{V}_{C}\right.$; $\left.\langle d \rho(A) x, y\rangle=0, A \in \mathscr{G}_{c}\right\}$ and $\left\{(x, y) \in \mathbb{T}^{*} \mathbb{V}_{c} ;\left\langle d \rho(A) \rho(g) x, \rho^{*}(g) y\right\rangle\right.$ $\left.=0, A \in \mathscr{G}_{c}\right\}$ coincide with each other. The characteristic variety $\mathfrak{S}$ decomposes into several $G_{C}$-orbits in $\mathbb{T}^{*} \mathbb{V}_{C}$. In fact, we can construct (5) as a union of a finite number of $G_{C}$-orbits in $\mathbb{T}^{*} \mathbb{V}_{C}$. In order to write it down, we begin with the orbital decomposition of $\mathbb{V}_{C}$.

The vector space $\mathbb{V}_{\boldsymbol{C}}=\boldsymbol{\operatorname { S y m }}(n, \mathbb{C})$ decomposes into $(n+1) \mathbb{G} \mathbb{L}(n, \mathbb{C})$ orbits $\mathbb{S}_{i C}=\{x \in \operatorname{Sym}(n, \mathbb{C}) ; \operatorname{rank}(x)=n-i\},(i=0,1, \ldots, n) . \quad$ The orbit $\mathbb{S}_{i c}$ is generated by $x_{i}=\left[\begin{array}{ll}I_{n-i} & \\ & 0_{i}\end{array}\right]$. Similarly, the vector space $\boldsymbol{V}_{\boldsymbol{C}}=\boldsymbol{M}(n, \boldsymbol{C}) \quad($ resp. $\boldsymbol{A} \boldsymbol{l} \boldsymbol{t}(2 n, \mathbb{C}))$ decomposes into $(n+1) \mathbb{G} \mathbb{L}(n, \mathbb{C}) \times$ $\mathbb{G L}(n, \boldsymbol{C})-(\operatorname{resp} . G \mathbb{L}(2 n, \mathbb{C})-)$ orbits $\mathbb{S}_{i C}=\{x \in \mathbb{M}(n, \mathbb{C}) ; \operatorname{rank}(x)=n-i\}$ (resp. $\left.\mathbb{S}_{i C}=\{x \in A l t(2 n, C) ; \operatorname{rank}(x)=2(n-i)\}\right),(i=0,1, \ldots, n)$. The orbit $S_{i c}$ is generated by $x_{i}=\left[\begin{array}{ll}I_{n-i} & \\ & 0_{i}\end{array}\right]$ (resp. $\left.x_{i}=\left[\begin{array}{ll}-I_{n-i} & \\ & 0_{2 i}\end{array}\right]\right)$. Here

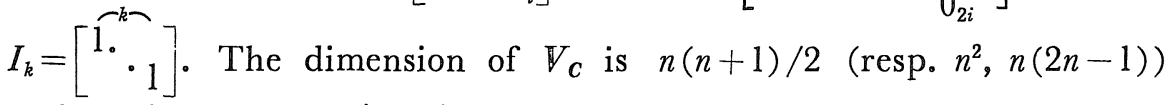
and we denote it by $n^{\prime}$, and the dimension of $\mathbb{S}_{i c}$ is $(n(n+1)-i(i+1)) / 2$ (resp. $n^{2}-i^{2}, n(2 n-1)-i(2 i-1)$ ) when $\mathbb{V}_{\boldsymbol{C}}=\mathbb{S} \mathbb{E} m(n, \mathbb{C}) \quad\left(\right.$ resp. $\mathbb{V}_{\boldsymbol{C}}=$ $\left.\mathbb{M}(n, \boldsymbol{C}), \boldsymbol{V}_{\boldsymbol{C}}=\boldsymbol{A} \boldsymbol{l t}(2 n, \boldsymbol{C})\right)$. The orbit $\mathbb{S}_{0 \boldsymbol{C}}$ is an open dense orbit in $\mathbb{V}_{C}$ and coincides with the set $\mathbb{V}_{C}-\left\{x \in \mathbb{V}_{C} ; P(x)=0\right\}$. The other orbits $\mathbb{S}_{i C}(i \geqq 1)$ are contained in the set $\left\{x \in \mathbb{V}_{C} ; P(x)=0\right\}$. The Zariski closure $\bar{S}_{i c}$ of $\mathbb{S}_{i c}$ is $\mathbb{S}_{i c} \cup \mathfrak{S}_{i+1 c} \cup \ldots \cup \mathbb{S}_{n c}$. 
We identify $T^{*} V_{c}^{*}$ and $V_{C} \times V_{c}^{*}$ in the same way as the identification of $\mathbb{T}^{*} V$ and $V_{C} \times V_{C}^{*}$. We denote by $\pi$ (resp. $\pi^{*}$ ) the projection map

$$
\pi ; V_{c} \times V_{C}^{*} \longrightarrow V_{c}\left(\operatorname{resp} . \pi^{*} ; \mathbb{V}_{c} \times V_{C}^{*} \longrightarrow V_{C}^{*}\right) .
$$

The maps $\pi$ and $\pi^{*}$ are compatible with the action of $G_{C}$.

Let $\boldsymbol{A}$ be a non-singular subvariety in $V_{c}$. We define the conormal bundle of $\boldsymbol{A}$ by

$$
\mathbb{T}_{A}^{*} \mathbb{V}_{C}=\bigcup_{x \in A}\left\{(x, y) \in V_{C} \times V_{C}^{*} ;\langle a, y\rangle=0 \text { for all } a \in \mathbb{T}_{x} \mathbb{A}\right\},
$$

which is clearly a non-singular subvariety of dimension $n^{\prime}$. If $\boldsymbol{A}$ is irreducible, then $\mathbb{T}_{\boldsymbol{A}}^{*} \boldsymbol{V}_{\boldsymbol{C}}$ is also irreducible. So the conormal bundle $\mathbb{T}_{\boldsymbol{S}_{i}}^{*} \boldsymbol{V}_{\boldsymbol{C}}$ is an irreducible variety, and hence $\overline{\boldsymbol{T}_{\boldsymbol{S}_{i}}^{*} \boldsymbol{V}_{\boldsymbol{C}}}$ is irreducible. We denote $\boldsymbol{\Lambda}_{i C}=\overline{\boldsymbol{T}_{S_{i}}^{*} \boldsymbol{V}_{\boldsymbol{C}}}$. Moreover we have $\pi\left(\mathscr{\Lambda}_{i C}\right)=\overline{\boldsymbol{S}}_{i}$. In the same way,

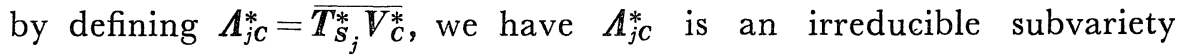
whose dimension is equal to $n^{\prime}$, and $\pi^{*}\left(\boldsymbol{\Lambda}_{j c}^{*}\right)=\overline{\boldsymbol{S}}_{j}$.

Let $\boldsymbol{\Sigma}_{i j C}$ be a $G_{C}$-orbit in $V_{C} \times \mathbb{V}_{C}^{*}$ generated by

$$
\left(\left[\begin{array}{cc}
I_{n-i} & \\
& 0_{i}
\end{array}\right],\left[\begin{array}{cc}
0_{j} & \\
& I_{n-j}
\end{array}\right]\right) \in V_{C} \times V_{C}^{*},
$$

when $\boldsymbol{V}_{\boldsymbol{C}}=\boldsymbol{S} \boldsymbol{y} \boldsymbol{m}(n, \boldsymbol{C})$ or $\mathbb{V}_{\boldsymbol{C}}=\mathbb{M}(n, \boldsymbol{C})$, and

$$
\left(\left[\begin{array}{ll}
J_{n-i} & \\
& 0_{2 i}
\end{array}\right],\left[\begin{array}{ll}
0_{2 j} & \\
& J_{n-j}
\end{array}\right]\right) \in \boldsymbol{V}_{\boldsymbol{C}} \times \boldsymbol{V}_{C}^{*},
$$

when $\boldsymbol{V}_{\boldsymbol{C}}=\boldsymbol{A l t}(2 n, \boldsymbol{C})$. Here, $J_{k}=\left[\begin{array}{c}I_{k} \\ -I_{k}\end{array}\right]$, and $n \geqq i \geqq 0, n \geqq j \geqq 0$ and $i+j \geqq n$. Then we have the orbital decompositions,

$$
\begin{aligned}
& \mathbb{T}_{\boldsymbol{S}_{i}}^{*} \boldsymbol{V}_{C}=\underset{j \geq n-i}{\cup} \boldsymbol{\Sigma}_{i j c}, \quad \mathbb{T}_{\boldsymbol{S}_{n-i}}^{*} \boldsymbol{V}_{\boldsymbol{C}}^{*}=\bigcup_{j \geq i} \boldsymbol{\Sigma}_{j n-i C}, \\
& \Lambda_{i c}=\underset{\substack{k \geq i \\
m \geqq n-i}}{\cup} \boldsymbol{\Sigma}_{k m C}, \quad \Lambda_{n-i C}^{*}=\underset{\substack{k \geq i \\
m \geqq n-i}}{\cup} \boldsymbol{\Sigma}_{k m C},
\end{aligned}
$$

and hence $\boldsymbol{\Lambda}_{i c}=\boldsymbol{\Lambda}_{n-i c}^{*}$. From the definition of the characteristic variety $\sqrt{ }$, $\mathfrak{S}$ is a closed set and coincides with $\underset{i \geqq 0}{\cup} \boldsymbol{T}_{\boldsymbol{S}_{i}}^{*} V_{C}$, and hence

$$
\mathfrak{E}=\overline{\bigcup_{i \geqq 0} T_{S_{i}}^{*} V_{c}}=\overline{\bigcup_{i \geqq 0} T_{S_{i}}^{*} V_{C}}
$$

Proposition 1.1. 1) $\mathbb{\Lambda}_{i C}=\mathbb{\Lambda}_{n-i c}^{*}$, and $\mathfrak{E}=\bigcup_{i \geq 0} \mathbb{\Lambda}_{i c}$.

2) The varieties $\boldsymbol{\Lambda}_{i c}$ and $\boldsymbol{\Lambda}_{i+1 c}$ have an intersection of dimension $\left(n^{\prime}-1\right)$ and it contains a $\boldsymbol{G}_{\boldsymbol{c}}$-orbit of dimension $\left(n^{\prime}-1\right)$. In a neighborhood of a point $p$ of the $\left(n^{\prime}-1\right)$-dimensional orbit, $\mathbb{\Lambda}_{i c}$ and $\mathbb{\Lambda}_{i+1 C}$ are smooth varieties and 


$$
\mathbb{T}_{p} \boldsymbol{\Lambda}_{i C} \cap \mathbb{T}_{p} \boldsymbol{\Lambda}_{i+1 C}=\mathbb{T}_{p}\left(\boldsymbol{\Lambda}_{i c} \cap \boldsymbol{\Lambda}_{i+1 c}\right) .
$$

Here, $\mathbb{T}_{\boldsymbol{D}} \mathbf{X}$ is the tangent space of at $p$.

3) $\mathbb{\Lambda}_{i c}$ and $\mathbb{\Lambda}_{j c}$ have no $\left(n^{\prime}-1\right)$-dimensional intersection if $j-i \geqq 2$.

The proof would be found in S-K-K-O [6].

We put $W_{C}$ the Zariski closure of the set

$$
\left\{\left(x, s \cdot \operatorname{grad}_{x} \log P(x)\right) \in \mathbb{V}_{c} \times \mathbb{V}_{c}^{*} ; x \in \mathbb{V}_{c}-\mathbb{S}_{c}, s \in \mathbb{C}\right\},
$$

and a conormal bundle $\boldsymbol{\Lambda}_{i c}$ is called a good Lagrangian subvariety if it is contained in $W_{C}$ and contains an open dense orbit in it. (See S-K-K-O [6], Definition 4.2, 4.5 and 4. 14). Furthermore, for a good Lagrangian subvariety $\mathbb{A}_{i}$, the order of $\mathfrak{M}_{s}$ is defined and is given by

$$
\operatorname{ord}_{A_{\imath} C}\left(\mathfrak{M}_{s}\right)=s \delta \chi\left(A_{0}\right)-t r_{v_{x_{i}}^{*}} d \rho^{*}\left(A_{0}\right)+{ }_{2}^{1} \operatorname{dim}_{C} \mathbb{V}_{x_{i}}^{*},
$$

where $A_{0}$ is an element of $\mathscr{G}_{0}$ satisfying $d \rho\left(A_{0}\right) x_{i}=0$ and $d \rho^{*}\left(A_{0}\right) y_{i}=y_{i}$ for an element $\left(x_{i}, y_{i}\right) \in \Sigma_{i, n-i c}$. Let $p$ be a point in $\mathfrak{E}$. We say that $\mathfrak{M}_{s}$ is a simple holonomic system at $p$ if:

(1.14) 1) $\operatorname{dim}_{C} \mathbb{E}=n$ and $\mathbb{E}$ is non-singular.

2) the $\mathcal{O}_{T^{*} V_{c}}$-ideal generated by $\left\{\langle d \rho(A) x, y\rangle ; A \in \mathscr{G}_{c}\right\}$ is a reduced ideal,

in a neighborhood of $p$. We say that two Lagrangian subvarieties $\mathbb{\Lambda}_{i c}$ and $\mathbb{\Lambda}_{j c}$ have a good intersection $\boldsymbol{\Sigma}$ if

(1.15) 1) $\boldsymbol{\Sigma}$ is a $\mathbb{G}_{C^{-}}$orbit in $\mathbb{A}_{i c}$ of dimension $n^{\prime}-1$,

2) $\mathbb{A}_{i c}, \mathbb{\Lambda}_{j c}$ and $\mathbb{W}_{c}$ are non-singular in a neighborhood of any point $p \in \boldsymbol{\Sigma}$.

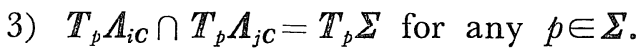

Proposition 1.2. 1) The variety $\boldsymbol{\Lambda}_{i c}$ are all good Lagrangian subvarieties.

2) The order of $\mathfrak{M}_{s}$ on $\boldsymbol{\Lambda}_{i c}$ is

$(1.16) \quad \operatorname{ord}_{\Lambda_{i C}}\left(\mathfrak{M}_{s}\right)= \begin{cases}-i s-\frac{i(i+1)}{4} & \left(\mathbb{V}_{c}=\operatorname{Sgm}(n, \mathbb{C})\right), \\ -i s-\frac{i}{2} & \left(\mathbb{V}_{C}=\mathbb{M}(n, \mathbb{C})\right), \\ -i s-\frac{i(2 i-1)}{2} & \left(\mathbb{V}_{c}=\mathbb{A} \mathbb{E}(n, \mathbb{C})\right),\end{cases}$

3) The Lagrangian subvarieties $\mathbb{\Lambda}_{i c}$ and $\mathbb{\Lambda}_{i+1 c}$ have a good intersection. 
The proof would be found in $\mathrm{S}-\mathrm{K}-\mathrm{K}-\mathrm{O}$ [6]. Now, by applying Theorems 6.3,6.6 and 8.3 in $\mathrm{S}-\mathrm{K}-\mathrm{K}-\mathrm{O}$, we have the following normal form of holonomic system near the point $z \in \boldsymbol{\Sigma}_{i+1, n-i c}$.

Theorem 1.3. Let $z \in \boldsymbol{\Sigma}_{i+1, n-i c}$. By a suitable quantized contact transformation, $\mathfrak{M}_{s}$ is transformed to the following holonomic system in a neighborhood of $z$.

$$
\begin{aligned}
& \left(x_{1} D_{x_{1}}-\lambda\right) u=0, \\
& \left(x_{2} D_{x_{2}}-\mu\right) u=0, \quad D_{x_{3}} u=\ldots=D_{x_{n}} u=0,
\end{aligned}
$$

with

$$
\begin{aligned}
& \mathbb{A}_{i+1 C}=\left\{(x, \xi) \in \mathbb{T}^{*} \mathbb{V}_{C} ; x_{1}=x_{2}=\xi_{3}=\ldots=\xi_{n^{\prime}}=0\right\} \\
& \mathbb{A}_{i C}=\left\{(x, \xi) \in \mathbb{T}^{*} \mathbb{V}_{C} ; x_{1}=\xi_{2}=\xi_{3}=\ldots=\xi_{n^{\prime}}=0\right\} \\
& \boldsymbol{Z}_{i+1 n-i C}=\mathbb{A}_{i+1 C} \cap \mathbb{A}_{i C}, \\
& z=\left(0, d x_{2}\right) \in \mathbb{T}^{*} \mathbb{V}_{C} \\
& \mu=\operatorname{ord}_{\Lambda_{i C}}\left(\mathfrak{M}_{s}\right)-\operatorname{ord}_{\Lambda_{i+1 C}}\left(\mathfrak{M}_{s}\right)-\frac{1}{2} \\
& = \begin{cases}s+\frac{i}{2} & \left(\mathbb{V}_{C}=\mathbb{S y m}(n, \mathbb{C})\right), \\
s+i & \left(\mathbb{V}_{C}=\mathbb{N}(n, C)\right), \\
s+2 i & \left(\mathbb{V}_{C}=\mathbb{A} \mathbb{E}(2 n, C)\right) .\end{cases}
\end{aligned}
$$

From those mentioned above, we have known the microlocal structures of holonomic systems $\mathfrak{M}_{s}$ on $\mathbb{T}^{*} \mathbb{V}_{c}$. It is simple on all the Lagrangian subvarieties and all the intersections of codimension one are good intersections.

Let $\mathfrak{M}_{s}$ be a holonomic system on an $n^{\prime}$-dimensional complex manifold $\mathbb{Z}_{C}$. We write a circle $\bigcirc$ to represent an irreducible Lagrangian component of $\mathrm{ch}\left(\mathfrak{M}_{s}\right)$ in $\mathbb{T}^{*} \mathbb{Z}_{C}$. We connect some of them by a line if and only if they have the same connected $n^{\prime}-1$ dimensional variety. We call the diagram thus obtained the complex holonomy diagram of $\mathfrak{M}_{s}$. We write the codimension of the projection of a Lagrangian subvariety in the circle. We write its order beside the good Lagrangian subvariety. When two Lagrangian subvarieties have a good intersection, we write $(\mu+1)$, which is defined in (1.18), beside the line connecting the two Lagrangian subvarieties.

Following to this definition, we have the complex holonomy diagrams in Figure 1. 

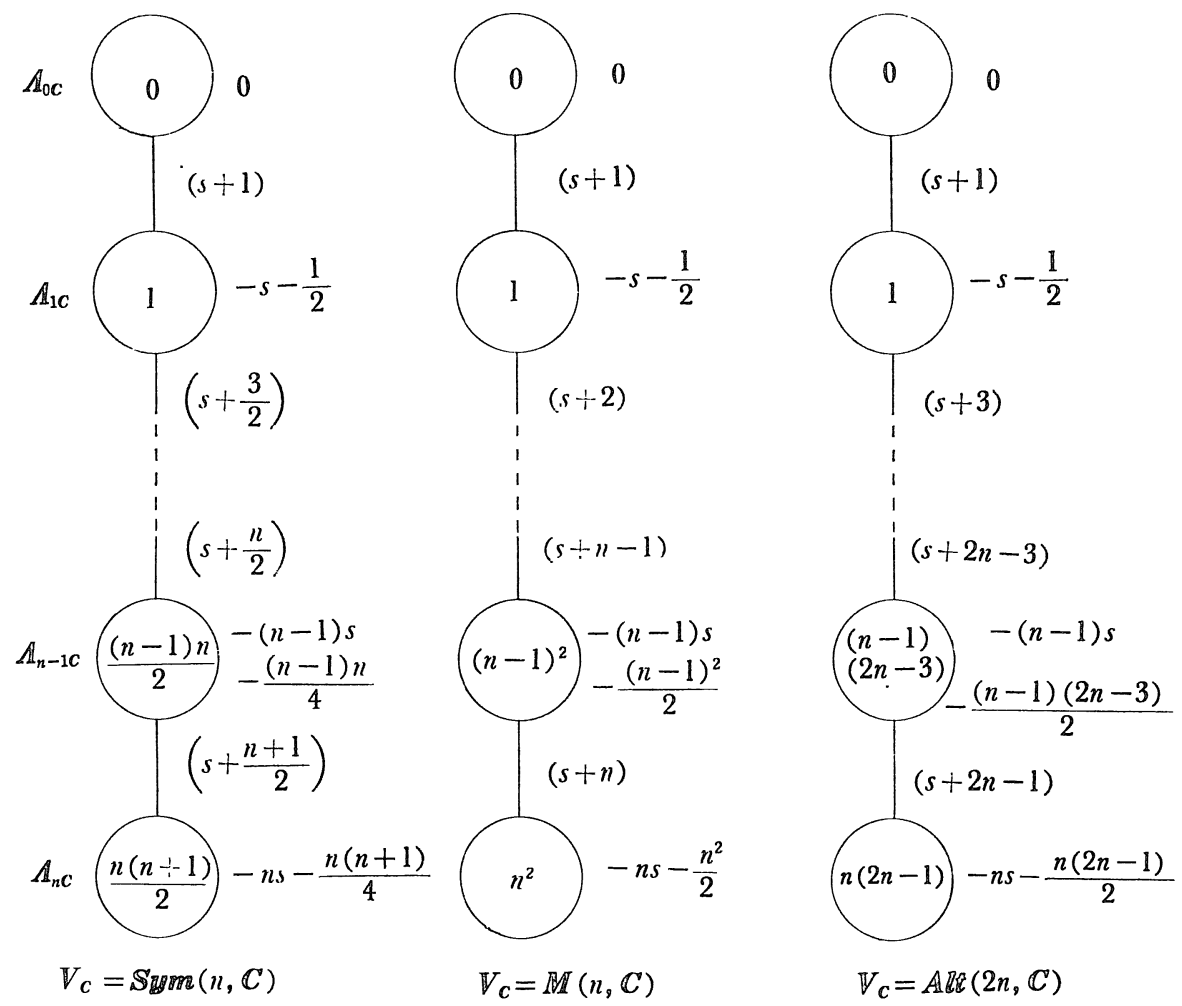

Figure 1 .

The microlocal structure of the holonomic system is completely determined by the difference of orders when two Lagrangian subvarieties have an intersection of codimension one. From the method of the calculations of $b$-functions in $\mathrm{S}-\mathrm{K}-\mathrm{K}-\mathrm{O}[6]$, we have the $b$-function of $P^{s}(x)$.

Proposition 1.4 1) There exists a polynomial $b(s)$, which we call a $b$-function, satisfying $P\left(D_{x}\right) \cdot P(x)^{s+1}=b(s) \cdot P(x)^{s}$.

2) (M. Kashiwara) The roots of $b(s)$ are negative rational numbers.

The proofs of this Proposition is found in Sato-Shintani[5] and $\mathrm{S}-\mathrm{K}-\mathrm{K}-\mathrm{O}[6]$ and Kashiwara[36]. 
Proposition 1.5. The b-functions of $P^{s}(x)$ are

1) $b(s)=\prod_{j=1}^{n}\left(s+\frac{1+j}{2}\right),\left(\boldsymbol{V}_{\boldsymbol{c}}=\boldsymbol{S y} \boldsymbol{m}(n, \boldsymbol{C})\right)$,

2) $b(s)=\prod_{j=1}^{n}(s+j), \quad\left(\boldsymbol{V}_{\boldsymbol{C}}=\mathbb{M}(n, \boldsymbol{C})\right)$,

3) $b(s)=\prod_{j=1}^{n}(s+2 j-1),\left(\mathbb{V}_{\boldsymbol{C}}=\boldsymbol{A l t}(2 n, \boldsymbol{C})\right)$,

modulo constant multiples.

Though the proof of this Theorem is possible by the direct computation, we obtain these by drawing the complex holonomy diagrams Figure 1. (See S-K-K-O[6], T. Kimura[24]).

\section{§2. Real Forms of Holonomic Systems and Principal Symbols (Applications of Kashiwara's Result)}

The main purpose of this section is essentially an application of Kashiwara's theory in [8] to some concrete examples. The results from Definition 2.1 to Theorem 2.6 may have been done in his computations [37]. The explanations after Theorem 2.6 to Theorem 2.12 are quoted from Kashiwara-Miwa [8] in a slightly different form for our use.

In this section, we shall consider the real forms of $\left(G_{C}, \rho, \mathbb{V}_{C}\right)$ and examine the holonomic system on the real locus.

We say that $\left(G_{R}, \rho, V_{R}\right)$ is a real form of $\left(G_{C}, \rho, \mathbb{V}_{C}\right)$ if $G_{R}$ is a real form of the complex Lie group $G_{C}, V_{R}$ is a real form of $V_{C}$ and the restriction of $\rho$ on $G_{R}$ is a representation of $G_{R}$ on $V_{R}$. We denote by $\boldsymbol{G}_{\boldsymbol{R}}^{+}$the connected component of $\mathbb{G}_{\boldsymbol{R}}$ containing the neutral element. We shall deal with $\left(G_{R}^{+}, \rho, V_{R}\right)$ in place of $\left(G_{R}, \rho, V_{R}\right)$ as a real form of $\left(G_{C}, \rho, V_{C}\right)$ if $G_{R}$ is not connected.

The followings are real forms of $\left(G_{C}, \rho, V_{C}\right)$ in (1.1)。

$$
\begin{aligned}
& \text { 1) } \boldsymbol{G}_{\boldsymbol{C}}=\boldsymbol{G L}(n, \boldsymbol{C}), \boldsymbol{V}_{\boldsymbol{C}}=\boldsymbol{S y \boldsymbol { m }}(n, \boldsymbol{C}) \\
& \text { (real symmetric bilinear forms) } \\
& \boldsymbol{G}_{\boldsymbol{R}}^{+}=\boldsymbol{G} \boldsymbol{L}(n, \boldsymbol{R})^{+} \ni g \\
& \boldsymbol{V}_{\boldsymbol{R}}=\boldsymbol{S y \boldsymbol { m }}(n, \boldsymbol{R})=\left\{x \in \boldsymbol{M}(n, \mathbb{R}) ;^{t} x=x\right\} \\
& \rho(g) ; x \longmapsto g \cdot x \cdot{ }^{t} g
\end{aligned}
$$


2) $G_{C}=G L(n, \mathbb{C}) \times \mathbb{G L}(n, \mathbb{C}), \mathbb{V}_{C}=\mathbb{M}(n, \mathbb{C})$

(complex Hermitian forms)

$G_{\boldsymbol{R}}=G L(n, \boldsymbol{C}) \ni g$

$\mathbb{V}_{\boldsymbol{R}}=\operatorname{Her}(n, \mathbb{C})=\left\{x \in \mathbb{M}(n, \mathbb{C}) ;{ }^{t} \bar{x}=x\right\}$

$\rho(g) ; x \longmapsto g \cdot x \cdot t \bar{g}$

3) $G_{\boldsymbol{C}}=G \mathbb{L}(2 n, \boldsymbol{C}), \mathbb{V}_{\boldsymbol{C}}=\operatorname{Alt}(2 n, \mathbb{C})$

(quaternion Hermitian forms)

$G_{R}=G \mathbb{L}(n, \mathbb{H}) \ni g$

$\boldsymbol{V}_{\boldsymbol{R}}=\boldsymbol{H e r}(n, \boldsymbol{H})=\left\{x \in \mathbb{M}(n, \mathbb{H}) ;^{t} \bar{x}=x\right\}$

$\rho(g) ; x \longmapsto g \bullet x \cdot t \bar{g}$

Here $\mathbb{H}$ stands for the quaternion field over $\mathbb{R}$. We regard the real Lie group $G_{R}$ (resp. the real vector space $\mathbb{V}_{R}$ ) as a sugroup of $G_{C}$ (resp. as a vector subspace of $V_{C}$ ) in the following way.

1) $G L(n, \mathbb{R}) \longrightarrow G L(n, \boldsymbol{C})$

$\Psi \quad U$

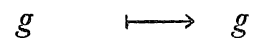

$\operatorname{Sym}(n, \boldsymbol{R}) \longrightarrow \operatorname{Sym}(n, \mathbb{C})$

$\Psi$

$x \quad \longmapsto \quad x$

2) $G L(n, C) \longrightarrow G L(n, \mathbb{C}) \times G L(n, \mathbb{C})$

$\Psi \quad U$

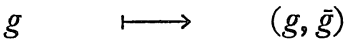

$\operatorname{Her}(n, \boldsymbol{C}) \longrightarrow \mathbb{M}(n, \mathbb{C})$

$\Psi \quad U$

$x \quad \longmapsto \quad x$

3) $\operatorname{GL}(n, \mathbb{H}) \longrightarrow G \mathbb{L}(2 n, \mathbb{C})$

$\Psi \quad U$

$g \quad \longmapsto \quad \iota(g)$

$\operatorname{Her}(n, \boldsymbol{H}) \longrightarrow \operatorname{Alt}(2 n, \mathbb{C})$

$U \quad U$

$\lambda \quad \longmapsto c(x) \cdot J_{n}$

Here $c$ is the map $\mathbb{H} \longrightarrow \mathbb{M}(2, \boldsymbol{C})$ defined by

$$
\iota ; x_{0} \cdot 1+x_{1} e_{1}+x_{2} e_{2}+x_{3} e_{1} e_{2} \longmapsto\left[\begin{array}{ll}
x_{0}+\sqrt{-1} x_{1}, & -x_{2}-\sqrt{-1} x_{3} \\
x_{2}-\sqrt{-1} x_{3}, & x_{0}-\sqrt{-1} x_{1}
\end{array}\right]
$$

where $e_{1}^{2}=e_{2}^{2}=-1, e_{1} e_{2}=-e_{2} e_{1}$, and $J_{n}$ is the $2 n \times 2 n$ alternative matrix defined by $c\left(-I_{n} e_{2}\right)$. We define the determinant of $x \in \mathbb{M}(n, \mathbb{H})$ by 
$\operatorname{det} x=\operatorname{Pff}\left(\iota(x) \cdot J_{n}\right)$.

The inner product $\langle$,$\rangle on \mathbb{V}_{\boldsymbol{R}}$ are defined by

$$
\begin{aligned}
& \text { 1) }\langle x, y\rangle=\operatorname{tr}\left(x^{t} y\right) \quad(x, y \in \boldsymbol{S y m}(n, \boldsymbol{R})) \\
& \text { 2) }\langle x, y\rangle=\operatorname{tr}\left(x^{t} \bar{y}\right) \quad(x, y \in \boldsymbol{H e r}(n, \boldsymbol{C})) \\
& \text { 3) }\langle x, y\rangle=\operatorname{tr}\left(x^{t} \bar{y}\right) \quad(x, y \in \boldsymbol{H e r}(n, \boldsymbol{H}))
\end{aligned}
$$

Note that the restrictions of the inner products by (2.3) coincide with the inner products in (2.4) up to constant multiples. We identify $\boldsymbol{V}_{\boldsymbol{R}}^{*}$ and $\boldsymbol{V}_{\boldsymbol{R}}$ by the inner products. Then $\boldsymbol{V}_{\boldsymbol{R}} \times \boldsymbol{V}_{\boldsymbol{R}}^{*}$ is a real form of the complex vector space $V_{\boldsymbol{C}} \times V_{\boldsymbol{c}}^{*}$ on which $G_{\boldsymbol{R}}^{+}$operates by $\rho \oplus \rho^{*}$ and the triple $\left(G_{R}^{+}, \rho^{*}, V_{R}^{*}\right)$ is a real form of $\left(G_{C}, \rho^{*}, V_{C}\right)$.

Let $x$ be a point in $\boldsymbol{V}_{\boldsymbol{R}}$. The real locus $\rho\left(\boldsymbol{G}_{C}\right) \cdot x \cap \mathbb{V}_{\boldsymbol{R}}$ is a $\boldsymbol{G}_{\boldsymbol{R}}^{+}$ invariant set and consists of a finite number of $G_{R}^{+}$-orbits. Each orbit is characterized by the signature of the orbit which is defined by the following.

Definition 2. 1. (Signatures of forms)

Let $x$ be an element of $\boldsymbol{V}_{\boldsymbol{R}}=\boldsymbol{S} \boldsymbol{y} \boldsymbol{m}(n, \boldsymbol{R})(\operatorname{resp} \boldsymbol{H} \boldsymbol{H e r}(n, \boldsymbol{C}), \boldsymbol{H e r}(n$, $\boldsymbol{H}))$. We say that a pair of non-negative integers $(k, m)$ is the signature of $x$, and denote is by $\operatorname{sign}(x)$ if there exist a basis $y_{1}, \ldots$, $y_{n} \in \mathbb{R}^{n}$ (resp. $\boldsymbol{C}^{n}, \boldsymbol{H}^{n}$ ) of the real vector space $\mathbb{R}^{n}$ (resp. the complex vector space $\boldsymbol{C}^{n}$, the left quaternion vector space $\boldsymbol{H}^{n}$ ) satisfying ${ }^{t} \bar{y}_{i} \cdot x \cdot y_{j}=\varepsilon_{i} \delta_{i j}$ with $\varepsilon_{i}=1,-1$ or 0 for $1 \leqq i \leqq k, k+1 \leqq i \leqq k+m$ or $i \geqq k+$ $m+1$, respectively $\left(\delta_{i j} ;\right.$ Kroneker's $\left.\delta\right)$. In particular, we say that $k$ (resp. $m$ ) is the positive (resp. negative) signature of $x$.

The following Proposition 2.2 is well know and Proposition 2.3 is easily verified.

\section{Proposition 2.2. (Orbital decompositions)}

The real vector space $\boldsymbol{V}_{\boldsymbol{R}}=\boldsymbol{S y m}(n, \boldsymbol{R}) \quad(\operatorname{resp} . \boldsymbol{H e r}(n, \boldsymbol{C}), \boldsymbol{H e r}(n, \boldsymbol{H}))$ decomposes into $\boldsymbol{G L}(n, \boldsymbol{R})^{+}$-orbits (resp. $\boldsymbol{G L}(n, \boldsymbol{C})$-orbits, $\boldsymbol{G L}(n, \boldsymbol{H})$ orbits),

$$
\begin{gathered}
\boldsymbol{S}_{i}^{j}=\{x \in \boldsymbol{S y m}(n, \boldsymbol{R}) \quad(\operatorname{resp} . \quad x \in \boldsymbol{H e r}(n, \boldsymbol{C}), \quad x \in \boldsymbol{H e r}(n, \boldsymbol{H})) \\
\operatorname{sign}(x)=(j, n-i-j)\}, \quad(i=0,1, \ldots, n, j=0, \ldots, n-i) .
\end{gathered}
$$


The orbit $\mathbb{S}_{i}^{j}$ is generated by $x_{i}^{(j)}=\left[\begin{array}{ll}I_{n-1}^{(j)} & \\ & 0_{i}\end{array}\right] \in \mathbb{V}_{R}$, where $I_{n-i}^{(j)}=\left[\begin{array}{ll}I_{j} & \\ & -I_{n-i-j}\end{array}\right]$. The real locus $\mathbb{S}_{i} \cap \mathbb{V}_{\boldsymbol{R}}=\underset{0 \leqq j \leqq n-i}{\cup} \boldsymbol{S}_{i}^{j}$.

In particular, among these $G_{\boldsymbol{R}}^{+}$-orbits, $\mathbb{S}_{0}^{j}(j=0, \ldots, n)$ are open orbits in $\mathbb{V}_{\boldsymbol{R}}$. We denote by $\boldsymbol{V}_{j}^{(n)}$ instead of $\mathbb{S}_{0}^{j}$. If $i>0$, then $\boldsymbol{S}_{i}^{j}$ is contained in the singular set $\left\{x \in \mathbb{V}_{R} ; P(x)=0\right\}$. We denote it by $\mathbb{S}_{R}$. We have

$$
\begin{aligned}
& \mathbb{V}_{n}^{(n)} \cup \mathbb{V}_{n-1}^{(n)} \cup \ldots \cup \mathbb{V}_{0}^{(n)}=\mathbb{V}_{R}-\mathbb{S}_{R}, \\
& \mathbb{S}_{R}=\underset{n \geqq i \geqq 1}{\cup} \mathbb{S}_{i R}, \mathbb{S}_{i R}=\bigcup_{n-i \geqq j \geqq 0} \mathbb{S}_{i}^{j} .
\end{aligned}
$$

Since the dual space $\mathbb{V}_{\boldsymbol{R}}^{*}$ is identified with $\mathbb{V}_{\boldsymbol{R}}$, and hence $\mathbb{V}_{\boldsymbol{R}}^{*}$ has the same decomposition. We denote them by

$$
\begin{aligned}
& \mathbb{V}_{n}^{*(n)} \cup \mathbb{V}_{n-1}^{*(n)} \cup \ldots \cup \mathbb{V}_{0}^{*(n)}=\mathbb{V}_{R}^{*}-\mathbb{S}_{R}^{*}, \\
& \mathbb{S}_{R}^{*}=\underset{n \geqq i \geqq 1}{\bigcup} \mathbb{S}_{i R}^{*}, S_{i R}^{*}=\underset{n-i \geqq j \geqq 0}{\bigcup} \mathbb{S}_{i}^{* j},
\end{aligned}
$$

when we have to distinguish $\mathbb{V}_{\boldsymbol{R}}$ from $\mathbb{V}_{\boldsymbol{R}}^{*}$. We say that $\mathbb{V}_{i}^{(n)}$ are open orbits, and that $\mathbb{S}_{i}^{j}$ are singular orbits.

Proposition 2. 3. Let $\boldsymbol{S}_{i}^{j}$ be the $\boldsymbol{G}_{\boldsymbol{R}}^{+}$-orbit defined in (2.6). Then we have

$$
\bar{S}_{i}^{j}=\bigcup_{\substack{n-i \geq p \geq 0 \\ \operatorname{Min}\{j, \bar{p}\} \geqq q \geqq \operatorname{Max}\{0, j-n+i+p !}} \mathbb{S}_{i+q}^{j-q}
$$

Now, consider the holonomic system $\mathfrak{M}_{s}$ in (1.4) and the hyperfunction solutions to $\mathfrak{M}_{s}$. Then the singular spectrum of the solution is contained in $\mathfrak{C}_{\boldsymbol{R}}=\operatorname{ch}\left(\mathfrak{M}_{s}\right) \cap \mathbb{T}^{*} \mathbb{V}_{\boldsymbol{R}}$. We may regard the solution as a microfunction whose support in contained in $\mathfrak{E}_{R}$. From Proposition 1. 1, we have $\mathfrak{E}=\cup_{i=0}^{n} \mathbb{A}_{i c}$ and

$$
\mathbb{A}_{i c}=\underset{\substack{j \geq n-i \\ k \geq i}}{\cup} \boldsymbol{\Sigma}_{k j c} \text { (disjoint union), }
$$

where $\boldsymbol{\Sigma}_{k j c}$ is the $\boldsymbol{G}_{\boldsymbol{C}}$-orbit in $\mathbb{V}_{\boldsymbol{C}} \times \mathbb{V}_{\boldsymbol{C}}^{*}$ generated by

$$
\left(\left[\begin{array}{ll}
I_{n-k} & \\
& 0_{k}
\end{array}\right],\left[\begin{array}{ll}
0_{j} & \\
& I_{n-j}
\end{array}\right]\right) \in \mathbb{V}_{R} \times \mathbb{V}_{R}^{*}
$$

Among the orbits in $\mathbb{A}_{i c}$, the orbit $\boldsymbol{Z}_{i, n-i c}$ is an open dense subset in $\boldsymbol{\Lambda}_{i c}$ and any point in $\boldsymbol{\Sigma}_{i, n-i c}$ does not contained in any other Lagrangian subvariety $\mathbb{A}_{j C}(j \neq i)$. Especially, we deonote by $\mathbb{A}_{i C}^{\circ}$ the $\mathbb{G}_{C}$-orbit 
$\boldsymbol{\Sigma}_{i, n-i c}$. We note that

$$
\widehat{\Lambda}_{i c}=\left(\underset{j \geq n-i}{\cup} \boldsymbol{\Sigma}_{i j c}\right) \cup\left(\cup_{j \geq i} \boldsymbol{\Sigma}_{j, n-i c}\right),
$$

and that $\hat{\Lambda}_{i c}$ is an open dense non-singular subvariety of $\boldsymbol{\Lambda}_{i c}$. (See Proposition 1.1). We have the following theorem on the real loci of the orbits $\boldsymbol{\Sigma}_{i j c}$.

The following Proposition 2.4 is fundamental in order to draw the real holonomy diagrams of $\mathfrak{M}_{s}$.

Proposition 2.4. 1) The real locus $\boldsymbol{\Sigma}_{i j \boldsymbol{R}}=\boldsymbol{\Sigma}_{i j \mathrm{c}} \cap V_{\boldsymbol{R}} \times V_{\boldsymbol{R}}^{*}$ decomposes into $(n-i+1) \times(n-j+1) \quad G_{R}^{+}$-orbits. The generators of $\boldsymbol{G}_{\boldsymbol{R}}^{+}$-orbits in $\boldsymbol{\Sigma}_{i j R}$ are

$$
\left(\left[\begin{array}{ll}
I_{n-i}^{(p)} & \\
& 0_{i}
\end{array}\right],\left[\begin{array}{ll}
0_{j} & \\
& I_{n-j}^{(q)}
\end{array}\right]\right) \in V_{R} \times V_{R}^{*} \quad\left(\begin{array}{l}
0 \leqq p \leqq n-i \\
0 \leqq q \leqq n-j
\end{array}\right) .
$$

We denote by $\boldsymbol{\Sigma}_{i j \boldsymbol{R}}^{p q}$ the $\boldsymbol{G}_{\boldsymbol{R}}^{+}$-orbits generated by (2.9). Especially, for $\boldsymbol{\Lambda}_{i \boldsymbol{R}}^{\circ}=\boldsymbol{\Sigma}_{i n-i \boldsymbol{R}}$, each connected component is a real Lagrangian submanifold. We denote $\boldsymbol{\Sigma}_{i n-i R}^{p q}$ by $\boldsymbol{\Lambda}_{i \boldsymbol{R}}^{p q}(0 \leqq p \leqq n-i, 0 \leqq q \leqq i)$.

2) The real locus $\hat{\boldsymbol{\Lambda}}_{i \boldsymbol{R}}=\hat{\boldsymbol{\Lambda}}_{i c} \cap\left(\boldsymbol{V}_{\boldsymbol{R}} \times \boldsymbol{V}_{\boldsymbol{R}}^{*}\right)$ is a non-singular subvariety. The intersection $\hat{\boldsymbol{\Lambda}}_{i \boldsymbol{R}} \cap \hat{\boldsymbol{\Lambda}}_{i+1 \boldsymbol{R}}$ consists of $\left(n^{\prime}-1\right)$-dimensional $\boldsymbol{G}_{\boldsymbol{R}}^{+}$-orbits and it is $\boldsymbol{\Sigma}_{i+1 n-i \boldsymbol{R}}$.

$$
\begin{aligned}
& \bar{\Lambda}_{i \boldsymbol{R}}^{p q} \cap\left(\hat{\boldsymbol{\Lambda}}_{i \boldsymbol{R}} \cap \hat{\boldsymbol{\Lambda}}_{i+1 \boldsymbol{R}}\right)=\boldsymbol{\Sigma}_{i+1, n-i \boldsymbol{R}}^{p q} \cup \boldsymbol{\Sigma}_{i+1 n-i \boldsymbol{R}}^{p-1 q} \\
& \overline{\boldsymbol{\Lambda}}_{i \boldsymbol{R}}^{p q} \cap\left(\hat{\boldsymbol{\Lambda}}_{i-1 \boldsymbol{R}} \cap \hat{\boldsymbol{\Lambda}}_{\boldsymbol{i} \boldsymbol{R}}\right)=\boldsymbol{\Sigma}_{i n-i+1 \boldsymbol{R}}^{p q-1} \cup \boldsymbol{\Sigma}_{i n-i+1 \boldsymbol{R}}^{p q} .
\end{aligned}
$$

Here we set $\boldsymbol{\Sigma}_{i j}^{p q}=\phi$ when $p>n-i$ or $q>n-j$.

Proof. 1) Let $u$ be a point in $\boldsymbol{\Sigma}_{i j R}$. Since $\pi(u) \in \boldsymbol{S}_{i R}, \pi(u)$ is reduced to one of the points

$$
x_{i}^{(p)}=\left[\begin{array}{ll}
I_{n-i}^{(p)} & \\
& 0_{i}
\end{array}\right], \quad(0 \leqq p \leqq n-i),
$$

by the actions of $\boldsymbol{G}_{\boldsymbol{R}}^{+}$. On the other hand, from the definition of $\boldsymbol{\Sigma}_{i_{\boldsymbol{k}}}$, we have $u \in \boldsymbol{T}_{\boldsymbol{S}_{i R}}^{* i} \boldsymbol{V}_{\boldsymbol{R}}$ and hence $\pi^{*}(u)$ is contained in the conormal vector space $V_{x_{i}}^{*}(p)$ of $S_{i R}$ at $x_{i}^{(p)}$. Let $G_{x_{i}}(p)$ be the isotropy subgroup of $\boldsymbol{G}_{\boldsymbol{R}}^{+}$at $x_{i}^{(p)}$. Then $\boldsymbol{G}_{x_{i}}(p)$ acts on $\boldsymbol{V}_{x_{i}}^{*}(p)$ by the contragredient representation, and the action $\rho_{x_{i}}^{*}(p)(g)$ is given in the following way. 


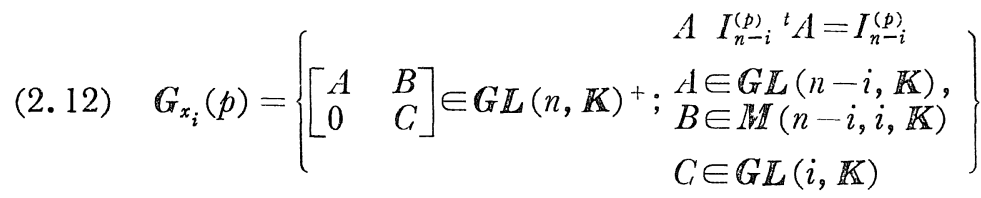

$$
\begin{aligned}
& \boldsymbol{V}_{x_{i}}^{*}(p)=\left\{\left[\begin{array}{ll}
0 & 0 \\
0 & X
\end{array}\right] \in \mathbb{V}_{\boldsymbol{R}}^{*}: X \in \mathbb{M}(i, K),{ }^{t} \bar{X}=X\right\}, \\
& \rho_{x_{i}}^{*}(p)(g) ;\left[\begin{array}{ll}
0 & 0 \\
0 & X
\end{array}\right] \longmapsto\left[\begin{array}{cc}
0 & 0 \\
0 & { }^{t} C^{-1} X C^{-1}
\end{array}\right], \\
& \text { with } g=\left[\begin{array}{ll}
A & B \\
0 & C
\end{array}\right] \in G_{x_{i}}(p) \text {. }
\end{aligned}
$$

Here, $\mathbb{K}$ is the field $\mathbb{R}$ (resp. $\mathbb{C}, \mathbb{H}$ ) when $\mathbb{V}_{\boldsymbol{R}}=\mathbb{S} \mathbb{R} \boldsymbol{m}(n, \mathbb{R})$ (resp. $\mathbb{H e r}(n, \mathbb{C}), \mathbb{H e r}(n, \mathbb{H}))$. From the assumption, the matrix $\left[\begin{array}{ll}0 & 0 \\ 0 & X\end{array}\right]$ is an element of $S_{j R}^{*}$ and hence $X$ is of rank $n-j$. Therefore it is reduced to the matrix,

$$
\left[\begin{array}{ll}
0_{i-n+j} & \\
& I_{n-j}^{(q)}
\end{array}\right]
$$

Thus we have that any point in $\Sigma_{i j R}$ is reduced to one of the points,

$$
\left(\left[\begin{array}{ll}
I_{n-i}^{(p)} & \\
& 0_{i}
\end{array}\right],\left[\begin{array}{ll}
0_{j} & \\
& I_{n-j}^{(q)}
\end{array}\right]\right) \in V_{R} \times \mathbb{V}_{R}^{*},\left(\begin{array}{l}
0 \leqq p \leqq n-i \\
0 \leqq q \leqq n-j
\end{array}\right) .
$$

Next we shall show that the $\mathbb{G}_{\boldsymbol{R}}^{+}$-orbits generated by the points (2.14) differ from one another. Let $u$ and $v$ be the points in $\boldsymbol{\Sigma}_{i j \boldsymbol{R}}$ given by

$$
\begin{aligned}
& u=\left(\left[\begin{array}{ll}
I_{n-i}^{(p)} & \\
& 0_{i}
\end{array}\right],\left[\begin{array}{ll}
0_{j} & \\
& I_{n-j}^{(q)}
\end{array}\right]\right), \\
& v=\left(\left[\begin{array}{ll}
I_{n-i}^{(r)} & \\
& 0_{i}
\end{array}\right],\left[\begin{array}{ll}
0_{j} & \\
& I_{n-j}^{(s)}
\end{array}\right]\right) .
\end{aligned}
$$

We assume that $p \neq r$ or $q \neq s$. Then we have that $\pi(u)$ and $\pi(v)$ are not contained in the same $G_{\boldsymbol{R}^{+}}^{+}$orbit or that $\pi^{*}(u)$ and $\pi^{*}(v)$ are not contained in the same $G_{R}^{+}$-orbit. Therefore, $G_{R^{*}}^{+} u$ and $G_{R}^{+}{ }^{\circ} v$ are not the same.

2) Since $\hat{\mathbb{A}}_{i C}$ is a non-singular variety, it is evident that $\hat{\mathbb{A}}_{i \boldsymbol{R}}$ is non-singular. The second statement follows from that $\hat{\mathbb{H}}_{i c} \cap \hat{\mathbb{A}}_{i+1 c}=$ $\Sigma_{i n-i+1 c}$.

3) First we shall show that

$$
\overline{\mathbb{A}}_{i R}^{p q} \supset \sum_{i+1 n-i R}^{p q} \cup \sum_{i+1 n-i R}^{p-1 q}
$$


Let $x$ be a point in $\sum_{i+1 n-i R}^{p q}$. Then $x$ is reduced to the point

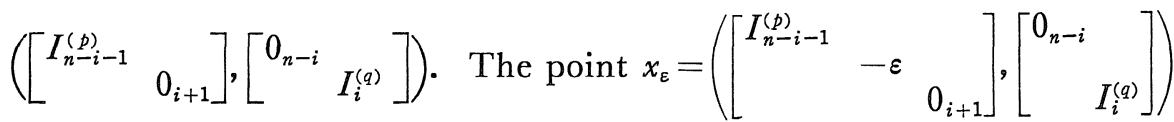
is an element in $\Lambda_{i R}^{p q}$ if $\varepsilon>0$, and converges to a point in $\sum_{i+1 n-i R}^{p q}$ when $\varepsilon \rightarrow 0$. Thus we have,

$$
\bar{\Lambda}_{i R}^{p q} \supset \sum_{i+1 n-i R}^{p q} .
$$

In the same way, $x_{\varepsilon}^{\prime}=\left(\left[\begin{array}{lll}I_{n-i-1}^{(p-1)} & & \\ & \varepsilon & \\ & & 0_{i}\end{array}\right],\left[\begin{array}{ll}0_{n-i} & \\ & I_{i}^{(q)}\end{array}\right]\right) \in \mathbb{M}_{i R}^{p q}$ for $\varepsilon>0$, and $x_{\varepsilon}^{\prime}$ converges to a point in $\sum_{i+1, n-i \boldsymbol{R}}^{p-1 q}$ when $\varepsilon \rightarrow 0$. Then we have

$$
\bar{\Lambda}_{i R}^{p q} \supset \sum_{i+1 n-i R}^{p-1 q} \text {. }
$$

Thus (2.16) is obtained.

Next we shall show that

$$
\bar{\Lambda}_{i R}^{p q} \cap \boldsymbol{\Sigma}_{i+1 n-i R}^{f g}=\phi \text {, if } f \neq p, p-1 \text { or } g \neq q .
$$

In fact, we have

and

$$
\pi\left(\bar{\Lambda}_{i R}^{p q}\right) \subset \bar{S}_{i}^{f}, \pi\left(\mathbb{Z}_{i+1 n-i R}^{f g}\right)=\$_{i+1}^{f},
$$

$$
\pi^{*}\left(\bar{\Lambda}_{i R}^{p q}\right) \subset \boldsymbol{S}_{n-i}^{* q}, \pi^{*}\left(\boldsymbol{\Sigma}_{i+1 n-i R}^{f g}\right)=\boldsymbol{S}_{n-i}^{* g}
$$

From Proposition 2.3, we have $\overline{\boldsymbol{S}}_{i}^{\phi} \cap \boldsymbol{S}_{i+1}^{f}=\phi$ if $f \neq p, p-1$ and $\boldsymbol{S}_{n-i}^{* g} \cap$ $\bar{S}_{n-i}^{* q}=\phi$ if $g \neq q$, and hence $(2.19)$ is followed. Thus we have

$$
\begin{aligned}
\bar{\Lambda}_{i R}^{p q} \cap\left(\hat{\boldsymbol{\Lambda}}_{i \boldsymbol{R}} \cap \hat{\boldsymbol{\Lambda}}_{i+1 \boldsymbol{R}}\right) & =\overline{\boldsymbol{\Lambda}_{i R}^{p q} \cap \boldsymbol{\Sigma}_{i+1 n-i R}} \\
& =\overline{\boldsymbol{\Sigma}}_{i+1 n-i \boldsymbol{R}}^{p q} \cup \boldsymbol{\Sigma}_{i+1 n-i R}^{p-1 q},
\end{aligned}
$$

and hence the first line of (2.10) is obtained.

By taking

$$
x_{\varepsilon}=\left(\left[\begin{array}{ll}
I_{n-i}^{(p)} & \\
& 0_{i}
\end{array}\right],\left[\begin{array}{lll}
0_{n-i} & & \\
& -\varepsilon & \\
& & I_{i-1}^{(q)}
\end{array}\right] \in \bar{\Lambda}_{i R}^{p q}(\varepsilon>0),\right.
$$

and

$$
x_{\varepsilon}^{\prime}=\left(\left[\begin{array}{ll}
I_{n-i}^{(p)} & \\
& 0_{i}
\end{array}\right],\left[\begin{array}{lll}
0_{n-i} & & \\
& \varepsilon & \\
& & I_{i-1}^{(q-1)}
\end{array}\right]\right) \in \bar{\Lambda}_{i R}^{\phi q} \quad(\varepsilon>0),
$$

and bringing $\varepsilon$ to zero, $x_{\varepsilon}$ and $x_{\varepsilon}^{\prime}$ converges to a point in $\boldsymbol{\Sigma}_{i n-i-1 \boldsymbol{R}}^{p q-1}$ and in $\sum_{i n-i+1 R}^{p q}$, respectively. On the other hand, we have

$$
\bar{\Lambda}_{i R}^{p q} \cap \sum_{i n-i+1 R}^{f g}=\phi \text { if } g \neq q, q-1 \text { or } f \neq p,
$$


because

$$
\begin{aligned}
& \pi\left(\overline{\mathbb{A}}_{i R}^{p q}\right) \subset \bar{S}_{i}^{p}, \pi\left(\mathbb{Z}_{i n-i+1 R}^{f g}\right)=\mathbb{S}_{i}^{f} \\
& \pi^{*}\left(\overline{\mathbb{A}}_{i R}^{p q}\right) \subset \bar{S}_{n-i}^{* q}, \pi^{*}\left(\sum_{i n-i+1 R}^{f g}\right)=\mathbb{S}_{n-i+1}^{* g}
\end{aligned}
$$

and

$$
\begin{aligned}
& \overline{\mathfrak{S}}_{i}^{p} \cap \mathbb{S}_{i}^{f}=\phi, \quad \text { if } f \neq p, \\
& \mathbb{S}_{n-i}^{* q} \cap \mathbb{S}_{n-i+1}^{* g}=\phi, \quad \text { if } g \neq q, q-1 .
\end{aligned}
$$

Thus we have

$$
\bar{\Lambda}_{i R}^{p q} \supset \sum_{i n-i+1 R}^{p q} \cup \sum_{i n-i+1 R}^{p q},
$$

and hence we obtain the second line of $(2.10)$.

$q \cdot e_{0} d$.

From Proposition 1.2, $\mathbb{A}_{i c}$ and $\mathbb{A}_{i+1 C}$ have a good intersection $\boldsymbol{\Sigma}_{i+1 n-i c}$. By restricting $\boldsymbol{\Sigma}_{i+1 n-i c}$ to the real domain, the real locus $\boldsymbol{\Sigma}_{i+1_{n-i \boldsymbol{R}}}$ decomposes into $(n-i) \times(i+1)-\mathbb{G}_{\boldsymbol{R}}^{+}$-orbits $\boldsymbol{\Sigma}_{i+1 n-i \boldsymbol{R}}^{p q}$ $(0 \leqq p \leqq n-i-1,0 \leqq q \leqq i)$. From Proposition 2.4-3), The $\mathbb{G}_{R}^{+}$-orbits in $\mathbb{A}_{i \boldsymbol{R}}$ which contains $\boldsymbol{\Sigma}_{i+1 n-i \boldsymbol{R}}^{p q}$ are $\mathbb{A}_{i \boldsymbol{R}}^{p+1 q}$ and $\mathbb{\Lambda}_{i \boldsymbol{R}}^{p q}$, and the $\mathbb{G}_{\boldsymbol{R}}^{+}$-orbits in $\mathbb{A}_{i+1 \boldsymbol{R}}$ which contains $\boldsymbol{\Sigma}_{i+1 n-i \boldsymbol{R}}^{p q}$ are $\mathbb{\Lambda}_{i+1 \boldsymbol{R}}^{p q+1}$ and $\mathbb{A}_{i+1 \boldsymbol{R}}^{p q}$. Thus we have the following proposition.

Proposition 2.5. Let $z=(x, y)$ be a point of $\mathbb{\Sigma}_{i+1 n-i R^{p}}^{p q}$ Then there exists a neighborhood $\mathbb{U}_{z}$ of $z$ in $\mathbb{T} * \mathbb{V}_{\boldsymbol{R}}$ such that

$$
\begin{aligned}
\mathbb{U}_{z} \cap \mathfrak{S}_{R} & =\left(\mathbb{A}_{i R} \cup \mathscr{A}_{i+1 R}\right) \cap \mathbb{U}_{z} \\
& =\left(\left(\mathbb{\Lambda}_{i R}^{p+1} \cup \mathscr{A}_{i R}^{p q}\right) \cup \mathbb{Z}_{i+1 n-i R}^{p q} \cup\left(\mathscr{H}_{i+1 R}^{p q+1} \cup \mathbb{H}_{i+1 R}^{p q}\right)\right) \cap \mathbb{U}_{z},
\end{aligned}
$$

in $\mathbb{U}_{z}$ 。

We write the Lagrangian subvarieties satisfying the conditions of Proposition 2.5 as

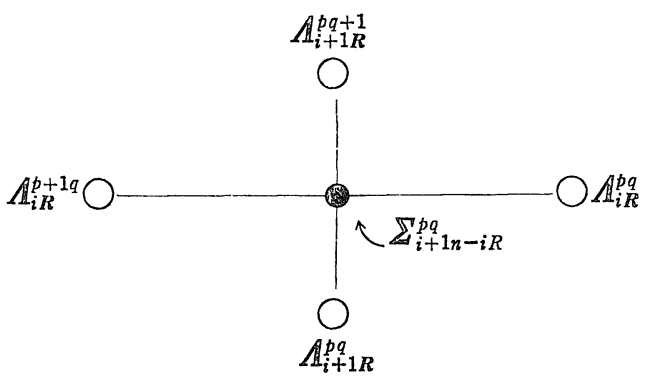

and call: it a real holonomy diagram. 
In the complex holonomy diagram, we write $\bigcirc$ to represent an irreducible Lagrangian component. But, in the real holonomy diagram, it means a connected component of the real locus of $\boldsymbol{\Lambda}_{i c}$. In our cases, each $\bigcirc$ is a $G_{R}^{+}$-orbit. By the diagram (2.25), we express the intersection in (Figure 2).

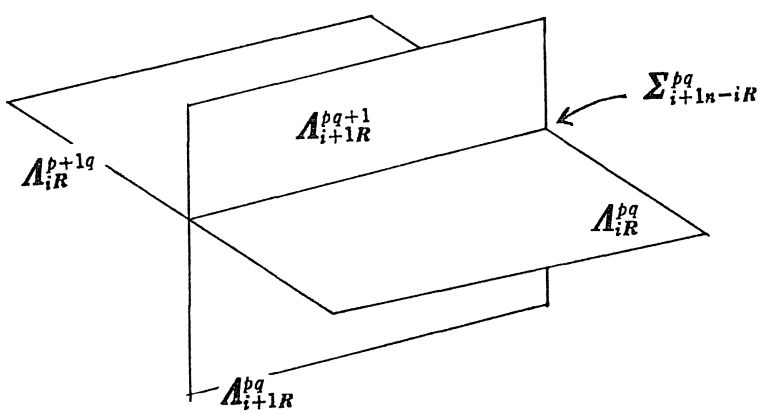

Figure 2.

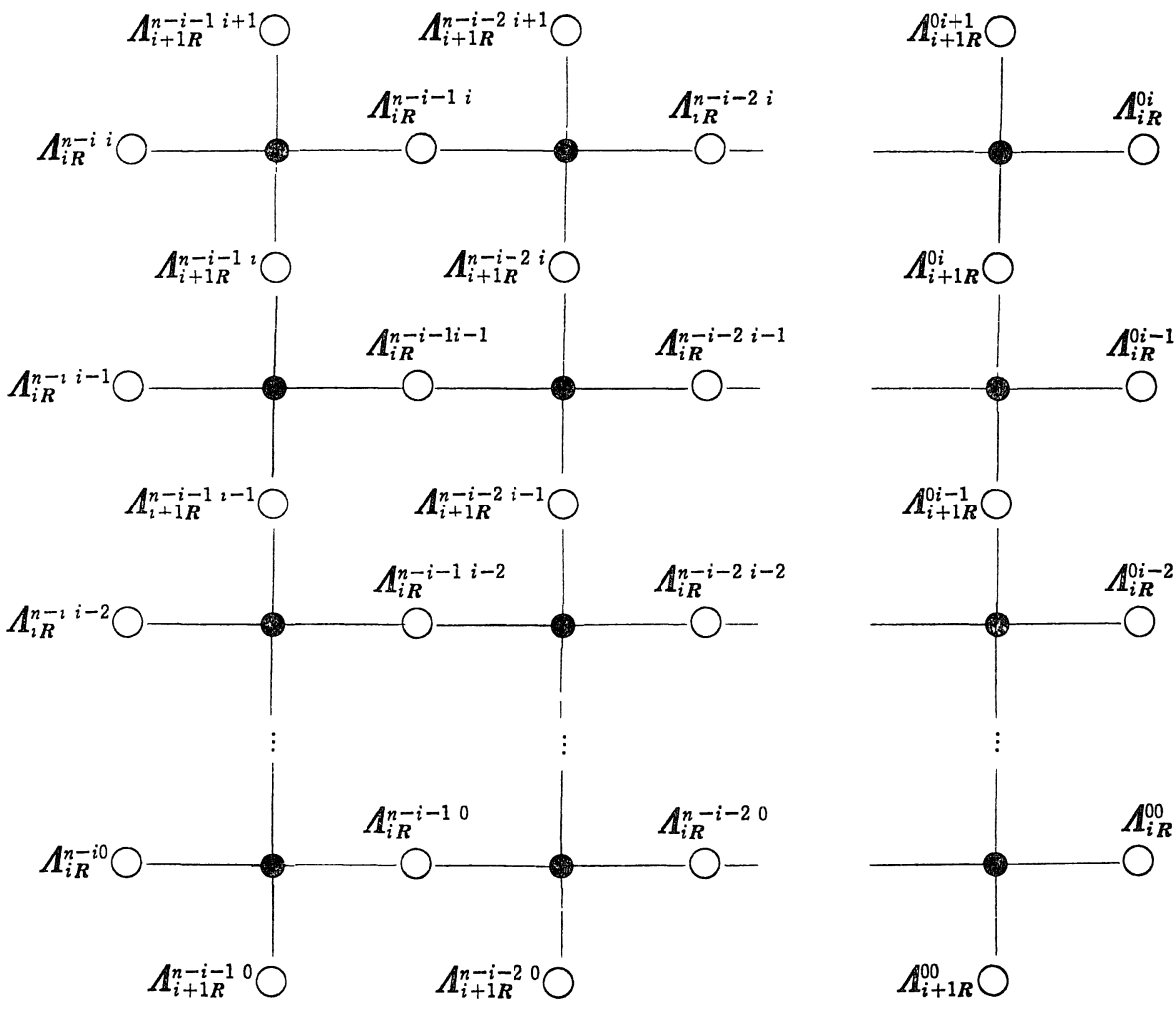

Figure 3. Real holonomy diagram of the intersection of $\boldsymbol{\Lambda}_{i \boldsymbol{R}}$ and $\boldsymbol{\Lambda}_{i+1 \boldsymbol{R}}$ 
The complete real holonomy diagram of intersections between $\mathbb{A}_{i R}$ and $\mathbb{\Lambda}_{i+1 R}$ is (Figure 3).

In Theorem 1.3, we have shown that, in a neighborhood of a point of $\boldsymbol{\Sigma}_{i+1 n-i c}$, the holonomic system $\mathfrak{M}_{s}$ is transformed to a holonomic system (1.17) by a suitable contact transformation. This contact transformation gives a real contact transformation in $\mathbb{T}^{*} \mathbb{V}_{R}$ and it transforms $\mathfrak{M}_{s}$ in the real locus.

Theorem 2.6. Let $z \in \sum_{i+1 n-i R^{p}}^{p q}$ By a suitable real quantized contact transformation, $\mathfrak{M}_{\mathrm{s}}$ is transformed to the following form in a neighborhood of $z$.

$$
\left\{\begin{array}{l}
\left(x_{1} D_{x_{1}}-\lambda\right) u=0, \\
\left(x_{2} D_{x_{2}}-\mu\right) u=0, D_{x_{3}} u=D_{x_{4}} u=\bullet=D_{x_{n}} u=0,
\end{array}\right.
$$

With

$$
\begin{aligned}
& \Lambda_{i+1 R}^{p+1}=\left\{(x, \xi) \in \mathbb{T}^{*} * V_{R} ; x_{1}=x_{2}=\xi_{3}=\circ=\xi_{n^{\prime}}=0, \xi_{2}>0\right\} \\
& \Lambda_{i+1 R}^{p q}=\left\{(x, \xi) \in \mathbb{T}^{*} V_{R} ; x_{1}=x_{2}=\xi_{3}=\circ=\xi_{n^{\prime}}=0, \xi_{2}<0\right\} \\
& \mathbb{\Lambda}_{i R}^{p+1 q}=\left\{(x, \xi) \in \mathbb{T}^{*} V_{R} ; x_{1}=\xi_{2}=\xi_{3}=\bullet=\xi_{n^{\prime}}=0, x_{2}>0\right\} \\
& \mathbb{A}_{i R}^{p q}=\left\{(x, \xi) \in T^{*} V_{R}, x_{1}=\xi_{2}=\xi_{3}=\cdots=\xi_{n^{\prime}}=0, x_{2}<0\right\} \\
& \mathbb{Z}_{i+1 n-i R}^{p q}=\left\{(x, \xi) \in \boldsymbol{T}^{*} V_{\boldsymbol{R}} ; x_{1}=x_{2}=\xi_{2}=\xi_{3}=\circ=\xi_{n^{\prime}}=0\right\} \\
& z=\left(0, d x_{2}\right) \in \mathbb{T}^{*} \mathbb{V}_{\boldsymbol{R}}, \mu= \begin{cases}s+\frac{i}{2} & \left(\mathbb{V}_{\boldsymbol{R}}=\operatorname{SWgm}(n, \mathbb{R})\right) \\
s+i & \left(\mathbb{V}_{\boldsymbol{R}}=\mathbb{H} \operatorname{Her}(n, \mathbb{C})\right) \\
s+2 i & \left(\mathbb{V}_{\boldsymbol{R}}=\mathbb{H} \operatorname{Her}(n, \mathbb{H})\right)\end{cases}
\end{aligned}
$$

Proof. In the following proof, we shall always consider all things in a sufficiently small neighborhood of $z$. The proof of the existence of the contact transformation of the holonomic system $\mathfrak{M}_{s}$ to the normal form (2.26) can be carried out in the same way as the proof of Theorem 1.3. In fact, our holonomic system $\mathfrak{M}_{s}$ has an involutory basis of micro-differential operators with real valued real analytic coefficients on $\mathbb{T}^{*} V_{R}$, and hence the contact transformation in $\mathbb{T}^{*} V_{C}$ defined in Theorem 1.3 preserves $\mathbb{T}^{*} \mathbb{V}_{R}$. Thus, there exists a contact coordinate transformation $\Psi$ which transforms $\mathfrak{M}_{s}$ to (2.26). That is to say, we can write,

$$
\begin{aligned}
& \Psi\left(\mathbb{\Lambda}_{i+1 R}\right)=\left\{(x, \xi) \in \mathbb{T}^{*} \mathbb{V}_{R} ; x_{1}=x_{2}=\xi_{3}=\circ=\xi_{n^{\prime}}=0\right\}, \\
& \Psi\left(\Lambda_{i R}\right)=\left\{(x, \xi) \in \mathbb{T}^{*} V_{R} ; x_{1}=\xi_{2}=\xi_{3}=\circ=\xi_{n^{\prime}}=0\right\},
\end{aligned}
$$




$$
\Psi(z)=\left(0, d x_{2}\right) \in T^{*} V_{R},
$$

and the holonomic system $\mathfrak{M}_{s}$ is written as (2.26). Thus we have only to show that if $\Psi\left(\boldsymbol{\Lambda}_{i \boldsymbol{R}}^{p+1 q}\right)=\Psi\left(\boldsymbol{\Lambda}_{i \boldsymbol{R}}\right) \cap\left\{x_{2}>0\right\}$, then $\phi\left(\boldsymbol{\Lambda}_{i+1 \boldsymbol{R}}^{p q+1}\right)=$ $\phi\left(\Lambda_{i+1 R}\right) \cap\left\{\xi_{2}>0\right\}$.

Let $f, g$ be real analytic, real valued functions such that

$$
\begin{aligned}
& d f(z) \neq 0, \quad\{f=0\} \supset \Lambda_{i+1 \boldsymbol{R}} \\
& d g(z) \neq 0, \quad\{g=0\} \supset \boldsymbol{\Lambda}_{i \boldsymbol{R}} .
\end{aligned}
$$

It is obvious that such functions exist. The Poisson bracket $\{f, g\}(z)$ does not vanish and we may assume that $\{f, g\}(z)>0$ by taking $-g$ instead of $g$ if necessary. Then we have

1) $\left.f\right|_{\boldsymbol{\Lambda}_{i \boldsymbol{R}}}$ has a zero of order one on $\boldsymbol{\Lambda}_{i \boldsymbol{R}} \cap \boldsymbol{\Lambda}_{i+1 \boldsymbol{R}}$, and does not vanish on $\boldsymbol{\Lambda}_{i R}-\left(\boldsymbol{\Lambda}_{i R} \cap \boldsymbol{\Lambda}_{i+1 R}\right)$.

2) $\left.g\right|_{\boldsymbol{\Lambda}_{i+1 \boldsymbol{R}}}$ has a zero of order one on $\boldsymbol{\Lambda}_{i R} \cap \boldsymbol{\Lambda}_{i+1 \boldsymbol{R}}$ and does not vanish on $\boldsymbol{\Lambda}_{i R}-\left(\boldsymbol{\Lambda}_{i R} \cap \boldsymbol{\Lambda}_{i+1 R}\right)$.

Then we have

(2.31) (the signature of $f$ on $\boldsymbol{\Lambda}_{i R}^{p+1 q}$ )

$\times\left(\right.$ the signature of $g$ on $\Lambda_{i+1 R}^{p q+1}$ )

does not depend on the choice of $f, g$. In fact, let $f^{\prime}, g^{\prime}$ be other real analytic, real valued functions satisfying (2.30) and $\left\{f^{\prime}, g^{\prime}\right\}(z)>0$. Then there exist real valued, real analytic functions $a, b$ which do not vanish at $z$ and satisfy

$$
\left.f^{\prime}\right|_{\Lambda_{i R}}=\left.a \cdot f\right|_{\Lambda_{i} R},\left.g^{\prime}\right|_{\Lambda_{i+1 R}}=\left.b \cdot g\right|_{\Lambda_{i+1 R}}
$$

We have

$$
\left\{f^{\prime}, g^{\prime}\right\}(z)=\{a f, b g\}(z)=a(z) b(z)\{f, g\}(z) .
$$

Therefore if $\left\{f^{\prime}, g^{\prime}\right\}(z)>0$, then $a(z) b(z)>0$ and hence we have $a b>0$ in a neighborhood of $z$. Thus we obtain that the signature of (2.31) is the same as the signature of (2.31) calculated by using $f^{\prime}, g^{\prime}$ instead of $f, g$.

We may take

$$
z=\left(\left[\begin{array}{ll}
I_{n-i-1}^{(p)} & \\
& 0_{i+1}
\end{array}\right],\left[\begin{array}{ll}
0_{n-i} & \\
& I_{i}^{(q)}
\end{array}\right]\right) \in T^{*} V_{R^{*}}
$$

\section{Setting}




$$
\begin{aligned}
& \phi_{1}(t)=\left(\left[\begin{array}{lll}
I_{n-i-1}^{(p)} & & \\
& t & \\
& & 0_{i}
\end{array}\right],\left[\begin{array}{ll}
0_{n-i} & \\
& I_{i}^{(q)}
\end{array}\right]\right) \\
& \psi_{2}(t)=\left(\left[\begin{array}{lll}
I_{n-i-1}^{(p)} & & \\
& 0_{i+1}
\end{array}\right],\left[\begin{array}{lll}
0_{n-i-1} & \\
& t & \\
& & I_{i}^{(q)}
\end{array}\right]\right)
\end{aligned}
$$

we have

$$
\begin{aligned}
& \phi_{1}(t) \in \boldsymbol{\Lambda}_{i R}^{p+1 q}, \psi_{2}(t) \in \Lambda_{i+1 R}^{p q+1}, \text { if } t>0, \\
& \psi_{1}(0)=\psi_{2}(0)=z .
\end{aligned}
$$

We can take $f, g$ satisfying

$$
\begin{aligned}
& f\left(\phi_{1}(t)\right)=t, \quad g\left(\phi_{2}(t)\right)=t, \\
& d f(z)=\left(\left[\begin{array}{lll}
0_{n-i-1} & & \\
& 1 & \\
& & 0_{i}
\end{array}\right],\left[\begin{array}{ll} 
& 0
\end{array}\right]\right)=\left(x_{1}, \xi_{1}\right) \in \mathbb{T}_{z}^{*}\left(\mathbb{T}^{*} \mathbb{V}_{R}\right) \\
& d g(z)=\left(\left[\begin{array}{ll}
0 & 0
\end{array}\right],\left[\begin{array}{ccc}
0_{n-i-1} & & \\
& 1 & \\
& & 0_{i}
\end{array}\right]\right)=\left(x_{2}, \xi_{2}\right) \in \mathbb{T}_{z}^{*}\left(\mathbb{T}^{*} \mathbb{V}_{R}\right) .
\end{aligned}
$$

Then we have $\left.\{f, g\}(z)=\left\{\left\langle x_{1}, \xi_{2}\right\rangle-\left\langle x_{2}, \xi_{1}\right\rangle\right\}=1\right\rangle 0$, and hence $f, g$ satisfy $\{f, g\}(z)>0$. We have

$$
\left.f\right|_{\boldsymbol{A}_{i \boldsymbol{R}}^{p+1} q}>0 \text { and }\left.g\right|_{\boldsymbol{A}_{i+1 \boldsymbol{R}}^{p q+1}}>0 \text {. }
$$

On the other hand, by the contact transformation, we may take

$$
f=x_{2} \text { and } g=\xi_{2} \text { 。 }
$$

In fact $\{f, g\}(\Psi(z))=1>0$. We have

$$
\begin{aligned}
& \left.x_{2}\right|_{\psi\left(\Lambda_{i R}\right) \cap\left\{x_{2}>0\right\}}>0, \\
& \left.\xi_{2}\right|_{\psi\left(\Lambda_{i+1 R}\right) \cap\left(\xi_{2}>0\right\}}>0 .
\end{aligned}
$$

From (2.38) and (2.40), if

$$
\Psi\left(\boldsymbol{\Lambda}_{i \boldsymbol{R}}^{p+1 q}\right)=\Psi\left(\boldsymbol{\Lambda}_{i R}\right) \cap\left\{x_{2}>0\right\},
$$

then

$$
\begin{aligned}
& \Psi\left(\boldsymbol{\Lambda}_{i R}^{p q}\right)=\Psi\left(\boldsymbol{\Lambda}_{i R}\right) \cap\left\{x_{2}<0\right\}, \\
& \Psi\left(\boldsymbol{\Lambda}_{i+1 R}^{p q+1}\right)=\Psi\left(\boldsymbol{\Lambda}_{i+1 R}\right) \cap\left\{\xi_{2}>0\right\} \\
& \Psi\left(\boldsymbol{\Lambda}_{i+1 \boldsymbol{R}}^{p q}\right)=\Psi\left(\boldsymbol{\Lambda}_{i+1 R}\right) \cap\left\{\xi_{2}<0\right\}
\end{aligned}
$$

Thus we have the result. q.e.d. 
In order to consider the hyperfunction solutions on $V_{R}$ to the holonomic system $\mathfrak{M}_{s}$ by "lifting up" $\mathfrak{M}_{s}$ on $\mathbb{T}^{*} V_{R}$, we introduce the real principal symbol of the microfunction solution. First, remember the definition of the principal symbol of a simple holonomic system. Let $\boldsymbol{X}$ be a real analytic manifold of dimension $n$ and let $\boldsymbol{X}_{C}$ be its complex neighborhood. Let $\mathfrak{M}$ be a holonomic system of differential equations on $\mathbb{X}_{C}$. It defines naturally $\mathscr{E}_{X} \otimes_{\pi^{-1}\left(\mathscr{D}_{X}\right)} \mathfrak{M}$ of microdifferential equations on $\mathbb{T}^{*} \mathbb{X}_{C}$. We also denote it by $\mathfrak{M}$ if there is no fear of confusion.

We denote by $\operatorname{ch}(\mathfrak{M})$ the characteristic variety of $\mathfrak{M}$. Let $\boldsymbol{\Lambda}_{C}$ be an irreducible component of $\operatorname{ch}(\mathfrak{M})$, and let $\mathbb{\Lambda}_{C}^{\circ}$ be the open subset of $\Lambda_{C}$ consisting of non-singular points in $\operatorname{ch}(\mathfrak{M})$. We suppose that $\mathfrak{M}$ is simple characteristic at any point of $\boldsymbol{\Lambda}_{C}^{\circ}$.

Now, remember the definition of complex principal symbols of $\mathfrak{M}$ on $\Lambda_{C}^{\circ}$. As in the definition 3.11. in S-K-K-O [6], we correspond a local holomorphic section of $\Omega_{\Lambda_{C}}^{\otimes 1 / 2} \otimes \Omega_{X_{C}}^{\otimes-1 / 2}$ on $\Lambda_{C}^{\circ}$ at each point to the holonomic system $\mathfrak{M}$. Here, $\Omega_{\Lambda_{C}}$ and $\Omega_{X_{C}}$ are the sheaves of holomorphic $n$-forms on $\Lambda_{c}$ and on $\boldsymbol{X}_{c}$, respectively. We call it the (complex) principal symbol of $\mathfrak{M}$ on $\mathbb{A}_{C}$. It is defined as a solution of a system of differential equations and it is defined modulo constant multiples. In other words, for a simple holonomic system $\mathfrak{M}$ on $\mathbb{A}_{C}^{\circ}$, we have a locally constant sheaf of rank one which is a subsheaf of $\Omega_{\Lambda_{C}}^{\otimes 1 / 2} \otimes \Omega_{X_{C}}^{\otimes-1 / 2}$ and the principal symbol is a local section of it. There does not always exists a global non-trivial section on $\mathbb{\Lambda}_{c}^{\circ}$.

We consider the hyperfunction or microfunction solutions to a holonomic system $\mathfrak{M}$. Let $\mathscr{B}_{\boldsymbol{X}}$ be the sheaf of hyperfunctions on $\mathbb{X}$. We denote by $\operatorname{Supp}(f(x))$ the support of a section $f(x) \in \mathscr{B}_{\boldsymbol{X}}$ on $\mathbb{X}$. We denote by S.S. $(f(x))$ the singular spectrum of $f(x)$ on $\mathbb{T}^{*} \boldsymbol{X}-\mathbf{X}$. Namely; for a section $f(x) \in \mathscr{B}_{X}$, the section $\operatorname{sp}(f(x))$ of the sheaf of microfunctions on $T^{*} \mathbb{X}-\mathbb{X}$ is defined. We denote it by $f(x)$ for simplicity. Then the support of $f(x)$ on $\mathbb{T}^{*} \boldsymbol{X}-\boldsymbol{X}$ is the singular spectrum on $T^{*}-\boldsymbol{X}$. Moreover, $f(x)$ is naturally considered as a section on $\mathbb{T}^{*} \boldsymbol{X}$ by corresponding $f(x)$ on $\boldsymbol{X}$ and $\operatorname{sp}(f(x))$ on $\mathbb{T} * \boldsymbol{X}-\boldsymbol{X}$. This section $f(x)$ on $\mathbb{T}^{*} \boldsymbol{X}$ is called a microfunction on $\mathbb{T}^{*} \boldsymbol{X}$. By this correspondence, any hyperfunction is naturally viewed as a microfunction on $T^{*} X$. We denote by S.S. $(f(x))$ the support of the microfunction $f(x)$ on $\mathbb{T}^{*} \boldsymbol{X}$. If $f(x)$ is a solution of a holonomic system $\mathfrak{M}$, 
then

$$
\text { S.S. }(f(x)) \subset \operatorname{ch}(\mathfrak{M}) \text {. }
$$

For any hyperfunction $f(x)$, we have

$$
\operatorname{Supp}(f(x))=\pi(\text { S.S. }(f(x))),
$$

where $\pi ; \mathbb{T}^{*} \boldsymbol{X} \rightarrow \boldsymbol{X}$ is the projection map.

Let $\mathbb{\Lambda}_{C}$ be an irreducible component of $\operatorname{ch}(\mathfrak{M})$. We suppose that $\mathbb{\Lambda}=\mathbb{\Lambda}_{\boldsymbol{C}} \cap \mathbb{T}^{*} \boldsymbol{X}$ is a real Lagrangian subvariety in $\mathbb{T}^{*} \mathbb{X}$. Let $z_{0}=\left(x_{0}, y_{0}\right)$ be a point in $\mathbb{1}^{\circ}=\mathbb{1}_{C}^{\circ} \cap \mathbb{T}^{*} \mathbb{X}$ and let $u$ be a microfunction solution to $\mathfrak{M}$ supported on $\mathbb{1}^{\circ}$ defined near $z_{0}$. We suppose that, in a neighborhood of $x_{0}$ in $\boldsymbol{X}, \pi\left(\mathscr{\Lambda}^{\circ}\right)=\left\{\tilde{x}_{1}=\cdots=\tilde{x}_{k}=0\right\}$ by a local coordinate $\left(\tilde{x}_{1}, \ldots, \tilde{x}_{k}\right.$, $\left.\tilde{x}_{k+1}, \ldots, \tilde{x}_{n}\right)$, and $\mathcal{\Lambda}$ is an open set in $\mathbb{T}_{\pi(\mathcal{A})}^{*} \mathbb{V}_{\boldsymbol{R}}=\left\{(\tilde{x}, \tilde{\xi}) \in \mathbb{T}^{*} \mathbb{V}_{\boldsymbol{R}} ; \tilde{x}_{1}=\bullet=\right.$ $\left.\tilde{x}_{k}=0, \tilde{\xi}_{k+1}=\cdots=\tilde{\xi}_{n}=0\right\}$. By using a microdifferential operator of fractional order (defined in $\mathrm{S}-\mathrm{K}-\mathrm{K}-\mathrm{O}[6] \S 2$ as $\left.\mathscr{E}_{V}^{\infty}(\lambda)\right) P=\sum_{j=0}^{\infty} P_{\lambda-j}\left(\tilde{x}^{\prime \prime}, \tilde{D}_{x}^{\prime}\right)$ defined in a neighborhood of $\left(x_{0}, y_{0}\right)$, we have an expression of $u$ near $\left(x_{0}, y_{0}\right)$,

$$
u=\int_{\left\{\left|\xi^{\prime}\right|=1\right\}} \sum_{j=0}^{\infty} P_{\lambda-j}\left(\tilde{x}^{\prime \prime}, \tilde{\xi}^{\prime}\right) \Phi_{\lambda-j+k}\left(\sqrt{-1}\left(\left\langle\tilde{x}^{\prime}, \tilde{\xi}^{\prime}\right\rangle+i 0\right)\right) d \omega\left(\tilde{\xi}^{\prime}\right) .
$$

This is the plane wave expansion of microfunction $u$ with respect to the coordinate $\left(x^{\prime}, \xi^{\prime}\right)$. Here, we set;

1) $\tilde{x}^{\prime}=\left(\tilde{x}_{1}, \ldots, \tilde{x}_{k}\right), \tilde{x}^{\prime \prime}=\left(\tilde{x}_{k+1}, \ldots, \tilde{x}_{n}\right)$ $\tilde{\xi}^{\prime}=\left(\tilde{\xi}_{1}, \ldots, \tilde{\xi}_{k}\right), \tilde{\xi}^{\prime \prime}=\left(\tilde{\xi}_{k+1}, \ldots, \tilde{\xi}_{n}\right)$.

2) $\Phi_{\lambda}(z)=\Gamma(\lambda)(-z)^{-\lambda}$. This function is defined for $-\pi+\varepsilon<\arg (z)$ $<\pi-\varepsilon$ and we take the branch satisfying $\Phi_{\lambda}(-1)=\Gamma(\lambda)$.

3) $d \omega\left(\tilde{\xi}^{\prime}\right)=\sum_{j=1}^{k}(-1)^{j} \tilde{\xi}_{j} d \tilde{\xi}_{1} \wedge . \wedge d \tilde{\xi}_{j-1} \wedge d \tilde{\xi}_{j+1} \wedge . \wedge d \tilde{\xi}_{k}$, is the measure on $(k-1)$ dimensional sphere $\left\{\left|\tilde{\xi}^{\prime}\right|=1\right\}$ 。

Let $\left|\Omega_{\Lambda}\right|$ (resp. $\left|\Omega_{X}\right|$ ) be the line bundle of the volume element on $\mathbb{A}$ (resp. $\mathbb{X})$. We can regard $\left|\Omega_{X}\right|$ as the line bundle on $\mathbb{A}$, whose transition function is defined by pulling back the transition function on $\boldsymbol{X}$ by the projection map $\pi$.

Definition 2.7. (Kashiwara) Let $u$ be a local section of a microfunction solution on $\mathbb{1}^{\circ}$ defined near $z_{0}$ expressed as (2.41). We define a local section $\sigma_{A}(u)$ of $\sqrt{\left|\Omega_{A}\right|} \times \sqrt{\left|\Omega_{X}\right|^{-1}}$ by 


$$
\sigma_{\boldsymbol{\Lambda}}(u)=(2 \pi)^{k / 2} P_{\lambda}\left(\tilde{x}^{\prime \prime}, \tilde{\xi}^{\prime}\right) \sqrt{\mid d \tilde{x}^{\prime \prime} \wedge d \tilde{\xi}^{\prime}} \mid / \sqrt{|d x|},
$$

and call it the real principal symbol of $u$. This definition does not depend on the choice of the local coordinate $(\tilde{x}, \tilde{\xi})$ on $\mathbb{T} * \boldsymbol{X}$.

The real principal symbol $(2.43)$ is obtained as a restriction of a complex principal symbol. Namely, let $z_{0}$ be a point in $\mathbb{1}^{\circ}$ and let $A$ be a non-trivial local section of complex principal symbol of the simple holonomic system $\mathfrak{M}$ defined near $z_{0}$. Then, for any microfunction solution $u$ defined near $z_{0}$, we have $\sigma_{\Lambda}(u)=\left.c \cdot A\right|_{\Lambda}$ with a constant $c$. This is easily verified by proving that $\sigma_{\Lambda}(u) \sqrt{|d x|}$ satisfies the differential equation for the principal symbol.

Conversely, let $A$ be a local section of a principal symbol on $\mathbb{1}^{\circ}$ defined near $z_{0}$. Then there exists a unique microfunction solution $u$ defined near $z_{0}$ such that $A=\sigma_{\Lambda}(u)$. In fact, let $\operatorname{Sol}(\mathfrak{M})_{z_{0}}$ be the vector space of microfunction solutions of $\mathfrak{M}$ near $z_{0}$ and let Symbol (MP $)_{z_{0}}$ be the vector space of principal symbols of $\mathfrak{M}$ near $z_{0}$. ThenSol $(\mathfrak{M})_{z_{0}}$ and Symbol( $\mathfrak{M})_{z_{0}}$ are one dimensional vector spaces over $C$ because $\mathfrak{M}$ is simple characteristic. Moreover,

$$
\begin{array}{ccc}
\sigma_{\Lambda} ; u & \longmapsto & \sigma_{\Lambda}(u) \\
\prod & \prod \\
\operatorname{Sol}(\mathfrak{M})_{z_{0}} & \longrightarrow \operatorname{Symbol}(\mathfrak{M})_{z_{0}},
\end{array}
$$

is a linear isomorphism. Thus, for a point $z_{0} \in \mathbb{1}^{\circ}$, if

(2.44) $\boldsymbol{\Lambda}$ is written as the conormal bundle of the non-singular subvariety $\pi(\mathbb{1})$ in a neighborhood of $z_{0}$,

then we have a one to one correspondence between $\operatorname{Sol}(\mathfrak{M})_{z_{0}}$ and Symbol(M $(\mathfrak{M})_{z_{0}}$ through the map $\sigma_{\Lambda^{*}}$. Henceforth, we suppose that

(2.45) for any point $z \in \Lambda^{\circ}$, the condition (2.44) is satisfied.

Let $\mathbb{1}^{1} \cup \ldots \cup \mathbb{A}^{k}=\mathbb{1}^{\circ}$ be the connected component decomposition. Let $\mathrm{Sol}(\mathfrak{M})\left(\mathbb{A}^{i}\right)$ be the vector space of global sections of microfunction solutions on $\mathscr{\Lambda}^{i}$ and let $\operatorname{Symbol}(\mathfrak{M})\left(\Lambda^{i}\right)$ be the vector space of global sections of principal symbols on $\mathscr{\Lambda}^{i}$. Since $\mathbb{\Lambda}^{i}$ is connected, $\operatorname{Sol}(\mathfrak{M})\left(\mathbb{A}^{i}\right)$ and $\operatorname{Symbol}(\mathfrak{M})\left(\mathbb{A}^{i}\right)$ are at most one-dimensional and there exists a one to one correspondence between them by the linear isomorphism $\sigma_{\boldsymbol{A}}$ for each $i$. Therefore any microfunction solution $u$ on $\mathbb{1}^{\circ}=$ 
$\mathbb{A}^{1} \cup \ldots \cup \mathbb{A}^{k}$ is determined by the global section of the real principal symbol $\sigma_{\boldsymbol{A}}(u)$ on $\mathbb{1}^{\circ}$. Namely, we have the following proposition.

Proposition 2. 8. Let $\mathfrak{M}$ be a holonomic system of differential equations with the characteristic variety $\operatorname{ch}(\mathfrak{M})=\cup_{i} \mathbb{A}_{i c}$ with $\mathbb{1}_{i c}$ an irreducible component. For each irreducible component $\mathbb{A}_{i c}$, we denote by $\mathbb{H}_{i c}^{\circ}$ the subset consisting of nonsingular points in $\operatorname{ch}(\mathfrak{M})$. We suppose that $\mathfrak{M}$ is simple on each $\mathbb{L}_{i c}^{\circ}$ and the condition $(2.45)$ is satisfied on $\mathbb{H}_{i}^{\circ}=\mathbb{H}_{i c}^{\circ} \cap \mathbb{T} * \mathbb{X}$. We put $\mathbb{A}_{i}^{1} \cup \ldots$ $\cup \mathbb{A}_{i}^{m_{i}}$ be the connected component decomposition of $\mathbb{H}_{i}^{\circ}$. Let $u(x)$ and $v(x)$ be two hyperfunction solutions to $\mathfrak{M}$. If their principal symbols $\sigma_{\Lambda_{i}}(u(x))$ and $\sigma_{\Lambda_{i}}(v(x))$ coincides with each other on every connected component $\mathbb{A}_{i}^{j}$, then $u(x)$ coincides with $v(x)$ as a microfunction on the subset $\cup_{i} \mathscr{M}_{i}^{\circ} \subset$ $\operatorname{ch}(\mathfrak{M})_{R}$.

Remark 2.9. 1) It is not yet proved that $u(x)$ coincides with $v(x)$ as a hyperfunction on $\mathbb{X}$. Later, we will prove that $u(x)$ actually coincides with $v(x)$ in some special cases. See Theorem 2. 14 .

2) Let $\mathfrak{M}$ be a holonomic system and let $\mathbb{R}$ be an irreducible component of $\operatorname{ch}(\mathfrak{M})$. For a microfunction solution $u(x)$ on $\mathbb{1}^{\circ}$, we do not yet have defined the real principal symbol $\sigma_{\Lambda}(u(x))$ at the point $z_{0} \in \mathbb{1}^{\circ}-\mathbb{A}_{\text {reg. }}^{\circ}$ Here, $\mathscr{H}_{\text {reg }}^{\circ}$ is the subset of the points satisfying the condition $(2.44)$. Then $\mathbb{L}_{\text {reg }}^{\circ}$ is an open dense subset of $\mathbb{H}^{\circ}$. In fact, we can not extend the real principal symbol on $\mathbb{A}_{\text {reg }}^{\circ}$ as a real analytic section on $\mathbb{1}^{\circ}$ in general. In order to correct the section on $\Lambda_{\mathrm{reg}}^{\circ}$ so as to be extendable real analytically to $\mathbb{1}^{\circ}$, we have to multiply a locally constant function on $\mathscr{\Lambda}_{\text {reg }}^{\circ}$ which is written by Maslov index. See for detail Kashiwara-Miwa [8].

Now, we go back to the case of the holonomic system $\mathfrak{M}_{s}$ :

$$
\left(\left\langle d \rho(A) x, D_{x}\right\rangle-s \delta \chi(A)\right) u=0 \text { 。 }
$$

Each Lagrangian subvariety $\mathscr{A}_{i c}$ in $\operatorname{ch}\left(\mathfrak{M}_{s}\right)$ is an irreducible component of $\operatorname{ch}\left(\mathfrak{M}_{s}\right)$ and $\mathbb{H}_{i c}^{\circ}=\boldsymbol{Z}_{i n-i c}$ is the subset of $\mathbb{L}_{i c}$ consisting of the non-singular points in $\operatorname{ch}\left(\mathfrak{M}_{s}\right)$. From Propositions 4.12 and 4.14 of $\mathrm{S}-\mathrm{K}-\mathrm{K}-\mathrm{O}$ [6], we have

Proposition 2. 10. The locally constant sheaf of complex principal symbol of $\mathfrak{M}_{s}$ on $\mathbb{H}_{i c}^{\circ}$ is generated by the nonzero section, 


$$
P_{\Lambda_{i C}}^{s} \sqrt{\omega_{\Lambda_{i C}}} / \sqrt{d x}
$$

with

$$
\begin{aligned}
& P_{\Lambda_{i C}}=P \circ \pi /\left.\sigma^{{ }^{m} \Lambda_{i}}\right|_{\Lambda_{i C}}, \\
& \omega_{\Lambda_{i C}}=\frac{\pi^{-1}(d x) \wedge d \sigma}{\sigma^{\mu_{\Lambda_{i}}}} /\left.d \sigma\right|_{\Lambda_{i C}} .
\end{aligned}
$$

Here,

$$
\begin{aligned}
& \sigma=\langle x, y\rangle / n, \\
& \pi ; T^{*} V_{c}=V_{c} \times V_{c}^{*} \supset \underset{c}{W_{c} \longrightarrow V_{c}^{*}} \\
& \underset{(x, y) \longrightarrow x}{U}
\end{aligned} \quad \text { (the projection map) }
$$

$d x=d x_{1} \wedge \ldots \wedge d x_{n^{\prime}}$ with a linear coordinate $\left(x_{1}, \ldots, x_{n^{\prime}}\right)$,

and $m_{\Lambda_{i}}$ and $\mu_{\Lambda_{i}}$ are the constants such that $-m_{\Lambda_{i}} s-\frac{\mu_{\Lambda_{i}}}{2}$ is the order of $\mathfrak{M}_{s}$ on $\Lambda_{i c}$.

Next, consider $\mathfrak{M}_{s}$ in the real domain. The real locus $\mathscr{\Lambda}_{i \boldsymbol{R}}^{\circ}=\mathbb{\Lambda}_{i C}^{\circ} \cap \mathbb{T}^{*} \boldsymbol{V}_{\boldsymbol{R}}$ is a real Lagrangian subvariety satisfying the condition (2.45), and has the connected component decomposition,

$$
\boldsymbol{\Lambda}_{i R}^{\circ}=\bigsqcup \Lambda_{i R}^{j k}, \quad\left(\begin{array}{l}
0 \leqq j \leqq n-i \\
0 \leqq k \leqq i
\end{array}\right),
$$

(see Proposition 2.4,1)). On each connected component $\Lambda_{i R}^{j k},\left|P_{\Lambda_{i}}\right|^{s}$ and $\sqrt{\left|\omega_{\Lambda_{i}}\right|}$ are a real analytic function and a real analytic half-volume element, respectively. Here, $P_{\Lambda_{i}}$ and $\omega_{\Lambda_{i}}$ are the restrictions of $P_{\Lambda_{i C}}$ and $\omega_{\Lambda_{i C}}$ to $\boldsymbol{\Lambda}_{i R}$. Then the section $\left|P_{\Lambda_{i}}\right| \sqrt[s]{\left|\omega_{\Lambda_{i}}\right|}$ gives a non-zero real analytic section of $\sqrt{\left|\Omega_{\Lambda_{i}}\right|}$ defined on $\Lambda_{i R}^{j k}$.

Proposision 2.11. On each connected component $\boldsymbol{\Lambda}_{i R}^{j k}$,

$$
\left|P_{\Lambda_{i}}\right| \sqrt[s]{\left|\omega_{\Lambda_{i}}\right|} / \sqrt{|d x|}
$$

is a basis of the vector space Symbol $\left(\mathfrak{M}_{s}\right)\left(\Lambda_{i R}^{j k}\right)$ of global sections of real principal symbols. Therefore, for any microfunction solution $u$ of $\mathfrak{M}_{s}$, the real principal symbol,

$$
\sigma_{\Lambda_{i R}^{j k}}(u)=c\left|P_{\Lambda_{i}}\right| s \sqrt{\left|\omega_{\Lambda_{i}}\right|}
$$


with a constant $c$.

Definition 2.12. (the coefficient or the associated number)

For a microfunction solution of $\mathfrak{M}_{s}$, we put

$$
\sigma_{\Lambda_{i R}^{p q}}(u)=c_{\boldsymbol{A}_{i R}^{p q}}\left|P_{\Lambda_{i}}\right| \sqrt[s]{\left|\omega_{\Lambda_{i}}\right|} / \sqrt{|d x|},
$$

and we call the constant $c_{\Lambda_{i R}^{p q}}$ the coefficient or the associated number of $u$ on $\Lambda_{i R}^{p q}$ with respect to the basis $\left|P_{\Lambda_{i}}\right| \sqrt{\left|\omega_{\Lambda_{i}}\right|} / \sqrt{|d x|}$. The constant term $c_{\boldsymbol{A}_{i \boldsymbol{R}}^{p q}}$ depends on the solution $u$, so we often denote it by $c_{\boldsymbol{A}_{\boldsymbol{i R}}^{p q}}(u)$.

Now, we have the following distinguished relations between the associated numbers. This theorem is obtained by an adaptation of the relation formula of real principal symbols in Kashiwara-Miwa [8] (p. 139, formula (3.5)).

Theorem 2. 13. (Relations of the coefficients)

$$
\begin{aligned}
& {\left[\begin{array}{l}
c_{\Lambda_{i+1 R}^{j-1 k+1}} \\
c_{\Lambda_{i+1}^{j-1 k}}
\end{array}\right]=\frac{\Gamma\left(s-s_{i+1}\right)}{\sqrt{2 \pi}}} \\
& \quad \times\left[\begin{array}{l}
\exp \left(-\frac{\pi}{2} \sqrt{-1}\left(s-s_{i+1}\right)\right), \exp \left(\frac{\pi}{2} \sqrt{-1}\left(s-s_{i+1}\right)\right) \\
\exp \left(\frac{\pi}{2} \sqrt{-1}\left(s-s_{i+1}\right)\right), \exp \left(-\frac{\pi}{2} \sqrt{-1}\left(s-s_{i+1}\right)\right)
\end{array}\right] \\
& \quad \times\left[\begin{array}{ll}
\exp \left(\frac{\pi}{4} \sqrt{-1}(i-2 k) v\right) & \exp \left(-\frac{\pi}{4} \sqrt{-1}(i-2 k) v\right)
\end{array}\right]\left[\begin{array}{c}
c_{\Lambda_{i R}^{j k}} \\
c_{\Lambda_{i \boldsymbol{R}}^{j-1 k}}
\end{array}\right]
\end{aligned}
$$

where

$$
\begin{aligned}
& s_{i}=\left\{\begin{array}{cl}
-(1+i) / 2 & \left(\mathbb{V}_{R}=\mathbb{S H m}(n, \mathbb{R})\right) \\
-i & \left(\mathbb{V}_{R}=\mathbb{H} \operatorname{er}(n, \mathbb{C})\right) \\
-2 i+1 & \left(\mathbb{V}_{R}=\mathbb{H} \operatorname{er}(n, \mathbb{H})\right)
\end{array}\right. \\
& v=\left\{\begin{array}{cl}
1 & \left(\mathbb{V}_{R}=\mathbb{S H m}(n, \mathbb{R})\right) \\
2 & \left(\mathbb{V}_{R}=\mathbb{H e r}(n, \mathbb{C})\right) \\
4 & \left(\mathbb{V}_{R}=\mathbb{H e r}(n, \mathbb{H})\right) .
\end{array}\right.
\end{aligned}
$$

Here, for $s=\left(s_{i+1}-k\right) \quad(k=0,1,2, \ldots)$ the matrix in $(2.49)$ is not well defined because the $\Gamma$-function has a pole. However, by computing 
the inverse matrix of it, we have,

$$
\begin{aligned}
& \frac{\Gamma\left(1+s_{i+1}-s\right)}{\sqrt{2 \pi}}\left[\begin{array}{l}
\exp \left(\frac{\pi}{2} \sqrt{-1}\left(1+s_{i+1}-s\right)\right), \exp \left(-\frac{\pi}{2} \sqrt{-1}\left(1+s_{i+1}-s\right)\right) \\
\exp \left(-\frac{\pi}{2} \sqrt{-1}\left(1+s_{i+1}-s\right)\right), \exp \left(\frac{\pi}{2} \sqrt{-1}\left(1+s_{i+1}-s\right)\right)
\end{array}\right] \\
& \times\left[\begin{array}{c}
c_{\Lambda_{i+1 R}^{j-1 k+1}} \\
c_{\Lambda_{i+1 R}^{j-1 k}}
\end{array}\right]=\left[\begin{array}{r}
\exp \left(\frac{\pi}{4} \sqrt{-1}(i-2 k) v\right), \\
\exp \left(-\frac{\pi}{4} \sqrt{-1}(i-2 k) v\right)
\end{array}\right]\left[\begin{array}{c}
c_{\Lambda_{i R}^{j k}} \\
c_{\Lambda_{i R}^{j-1 k}}
\end{array}\right],
\end{aligned}
$$

For the number $s=\left(s_{i+1}-k\right)(k=0,1,2, \ldots),(2.51)$ is well defined and we interpret the relation matrix $(2.49)$ as the relation matrix (2.51). Then the relations among the coefficients $\left\{c_{\Lambda_{i R}^{j k}}\right\}$ are well defined for all $s \in \boldsymbol{C}$.

Let $\left\{c_{i}^{j k}\right\}$ be a set of the coefficients on $\left\{\boldsymbol{\Lambda}_{i R}^{j k}\right\}$ which are compatible with the relation matrices (2.49), i. e., $\left\{c_{i}^{j k}\right\}$ satisfies the relations defined by (2.49). Then, by Proposition 2.8, there exists a unique microfunction solution $u(x)$ on $\cup_{i=0}^{n} \Lambda_{i}^{\circ} \subset \operatorname{ch}\left(\mathfrak{M}_{s}\right)_{R}$ whose coefficients $c_{\boldsymbol{A}_{i \boldsymbol{R}}^{j k}}(u(x))$ on $\boldsymbol{\Lambda}_{i \boldsymbol{R}}^{j k}$ are $c_{i}^{j k}$. However, this statement does not give guarantee for the existence of the microfunction solution to $\mathfrak{M}_{s}$ on $\operatorname{ch}\left(\mathfrak{M}_{s}\right)_{\boldsymbol{R}}=\cup_{i=0}^{n} \mathbb{\Lambda}_{i}$ whose coefficients on $\boldsymbol{\Lambda}_{i \boldsymbol{R}}^{j k}$ are $c_{i}^{j k}$. If the existence of the microfunction solution globally defined on $\operatorname{ch}\left(\mathfrak{M}_{s}\right)_{R}$ is proved, then it means the existence of the hyperfunction solution on $V_{R}$. In the next section, we shall show the global existence of the hyperfunction solution on $V_{R}$.

We conclude this section by showing the uniqueness of the hyperfunction solution, i. e., if there exists a hyperfunction solution $u(x)$ such that $c_{\boldsymbol{A}_{i \boldsymbol{R}}^{j k}}(u(x))=c_{i}^{j k}$, then such hyperfunction solution is uniquely determined. Namely, we have the following theorem.

Theorem 2.14. Let $u_{1}(x)$ and $u_{2}(x)$ be two hyperfunction solutions to the holonomic system $\mathfrak{M}_{s}$. Then the following three conditions are equivalent.

i) $u_{1}(x)=u_{2}(x)$.

ii) The real principal symbol $\sigma_{\Lambda_{i}}\left(u_{1}(x)\right)$ and $\sigma_{\boldsymbol{A}_{i}}\left(u_{2}(x)\right)$ coincides 
with each other on every connected component $\mathbb{A}_{i R}^{j}$.

iii) $c_{\boldsymbol{A}_{i R}^{j k}}\left(u_{1}(x)\right)=c_{\boldsymbol{A}_{i R}^{j k}}\left(u_{2}(x)\right)$ for every $\mathbb{\Lambda}_{i R}^{j k}$.

Proof. The conditions ii) and iii) are appearently equivalent. The condition i) clearly implies ii). We shall show the converse ii) $\Rightarrow$ i). Note the following lemma.

Lemma 2.15. (Holmgren's type theorem) Let $\mathbb{X}$ be a real analytic manifold and let $u(x)$ be a hyperfunction defined near a point $x_{0} \in \mathbb{X}$. Let $p(x)$ be a real valued real analytic function defined near $x_{0}$ such that $p\left(x_{0}\right)=0$ and $d p\left(x_{0}\right) \neq 0$. We suppose that

i) $\operatorname{Supp}(u(x)) \subseteq\{p(x) \geqq 0\}$,

ii) S. S. $(u(x)) \nsubseteq\left(x_{0}, d p\left(x_{0}\right)\right)$ or S. S. $(u(x)) \nsupseteq\left(x_{0},-d p\left(x_{0}\right)\right)$.

Here, S. S. $(u(x))$ means the singular spectrum of $u(x)$ in $\mathbb{T} * \mathbb{X}-\mathbb{X}$. Then we have $u(x)=0$ near $x_{0}$.

The proof of this lemma is given in S-K-K [7] p. 471 Proposition 2. 1.3 and the next remark.

Corollary 2.16. Let $X$ be a real analytic manifold and let $\mathbb{Y}$ be a non-singular real analytic subvariety in $\mathbb{X}$ near $x_{0} \in \mathbb{X}$. Let $u(x)$ be a hyperfunction defined near $x_{0}$. We suppose that

1) $\operatorname{Supp}(u(x)) \subseteq \mathbb{Y}$.

2) S. S. $(u(x)) \not\left(\mathbb{T}_{Y}^{*} \mathbb{X}-\mathbb{X}\right) \cap \pi^{-1}\left(x_{0}\right)$ 。

Here $\pi$ is the projection map $\mathbb{T} * \mathbb{X} \rightarrow \mathbb{X}$. Then $u(x)=0$ near $x_{0}$.

Proof. There exists a local coordinate $\left(p_{1}, \ldots, p_{n}\right)$ difined near $x_{0}$ such that $Y=\left\{p_{1}=\cdots=p_{m}=0\right\}$, and $p_{i}\left(x_{0}\right)=0$. From the condition 2), there exists a point $\left(x_{0}, y_{0}\right) \in\left(\mathbb{T}^{*} \mathbb{X}-\mathbb{X}\right)$ such that $\left(x_{0}, y_{0}\right) \notin \mathrm{S}$. S. $(u(x))$. We can take $p(x)=\sum_{i=1}^{m} c_{i} p_{i}(x)$ with $c_{i} \in \mathbb{R}$ such that $d p\left(x_{0}\right)=y_{0}$. Then the condition (2.53) i), ii) are satisfied. Thus we have the result.

Now we go to the proof of the theorem. We put $v(x)=u_{1}(x)-$ $u_{2}(x)$. Since $v(x)$ is a hyperfunction solution to $\mathfrak{M}_{s}$, we have 
S. S. $(v(x)) \subset\left(\operatorname{ch}\left(\mathfrak{M}_{s}\right) \cap T^{*} V_{R}\right)-V_{R}=\cup_{i=1}^{n}\left(\overline{T_{S_{i R}^{*}}^{*} V_{R}}-V_{R}\right)$, by (2.41). On the other hand, $v(x)=u_{1}(x)-u_{2}(x)=0$ on $\boldsymbol{V}_{\boldsymbol{R}}-\boldsymbol{S}_{\boldsymbol{R}}$ because $\sigma_{\Lambda_{0}}\left(u_{1}(x)\right)=\sigma_{\Lambda_{0}}\left(u_{2}(x)\right)$ on $V_{R}-S_{R} \times\{0\} \subset \mathbb{T}^{*} V_{R}$. Therefore, $\operatorname{Supp}(v(x)) \subset \pi\left(\cup_{i \geqq 1} \boldsymbol{\Lambda}_{i}\right)=\boldsymbol{S}_{R}=\boldsymbol{S}_{1 R} \cup \boldsymbol{S}_{2 R} \cup \ldots \cup \boldsymbol{S}_{n R}$,

by (2. 42$)$.

We shall prove that $v(x)=0$ by induction. Suppose that (2.54) $\quad \operatorname{Supp}(v(x)) \subset \overline{\boldsymbol{S}}_{i \boldsymbol{R}}=\boldsymbol{S}_{i R} \cup \ldots \cup \boldsymbol{S}_{n \boldsymbol{R}}$.

Then, for any point $x_{0} \in \boldsymbol{S}_{i \boldsymbol{R}}, \boldsymbol{S}_{\boldsymbol{i} \boldsymbol{R}}$ is a non-singular subvariety near $x_{0}$. Since the real principal symbol $\sigma_{\Lambda_{i}}(v(x))=0$ on $\boldsymbol{\Lambda}_{i}^{\circ}$, we have

$$
\text { S. S. }(v(x)) \cap \boldsymbol{\Lambda}_{i}^{\circ}=\phi \text {. }
$$

The variety $\boldsymbol{\Lambda}_{i}^{\circ}$ is an open dense subset of $\boldsymbol{\Lambda}_{i}={\overline{T_{S}}}_{\boldsymbol{S}_{\boldsymbol{R}}}^{*} \boldsymbol{V}_{\boldsymbol{R}}$ and hence

$$
\text { S. S. }(v(x)) \supset T_{S_{i R}}^{*} V_{R} \cap \pi^{-1}\left(x_{0}\right) \supset \Lambda_{i}^{\circ} \cap \pi^{-1}\left(x_{0}\right) \text {. }
$$

Thus, by Corollary 2.16, we have $v(x)=0$ near $x_{0}$, and hence we have

$$
\operatorname{Supp}(\nu(x)) \subset \bar{S}_{i+1 R}=S_{i+1 R} \cup \ldots \cup \boldsymbol{S}_{n R} .
$$

Then, by induction on $i$, we have $\operatorname{Supp}(v(x))=\phi$, i. e., $v(x)=0$ on $\boldsymbol{V}_{\boldsymbol{R}}$. q. e. d.

\section{Chapter II. Constructions of Relatively Invariant Hyperfunctions and the Fourier Transforms}

The purpose of this chapter is to construct some hyperfunction solutions to $\mathfrak{M}_{s}$ and to calculate the Fourier transforms of them. The results of the Fourier transforms in $\S 3$ were first computed by $M$. Sato and T. Shintani [5] and T. Shintani [21] by another method when $\boldsymbol{V}_{\boldsymbol{R}}=\boldsymbol{H e r}(n, \boldsymbol{C})$ and $\boldsymbol{V}_{\boldsymbol{R}}=\boldsymbol{S} \boldsymbol{y} \boldsymbol{m}(n, \mathbb{R})$, respectively. As far as the results in Theorem 3.9, T. Suzuki has obtained them by using Kashiwara's method. But he did not state nothing about the results after Theorem 3. 10 。

The coefficients of real principal symbols on a Lagrangian subvariety is always with respect to the basis in (2.47). In this chapter, we shall always deal with the real forms, so we often omit $\mathbb{R}$ beside the notations. 
For example, we denote simply $\mathbb{V}$ instead of $\mathbb{V}_{R}$.

\section{§3. The Hyperfunctions $|P|_{i}^{s}(x)$ and Their Fourier Transforms}

We begin with the definition of tempered distributions with meromorphic parameter $s \in \mathbb{C}$.

Definition 3.1. Let $\Omega$ be a domain in $\mathbb{C}$ and let $X$ be a finite dimensional real vector space.

1) We say that $u_{s}(x)$ is a tempered distribution with a holomorphic parameter $s \in \Omega$ if

i) For any $s \in \Omega, u_{s}(x)$ is a tempered distribution on $\boldsymbol{X}$.

ii) For any $f \in \mathscr{S}(\mathbb{X}), T_{s}(f)=\int u_{s}(x) f(x) d x$ is holomorphic in $s \in \Omega$.

2) We say that $u_{s}(x)$ is a tempered distribution with a meromorphic parameter $s \in \Omega$ if $u_{s}(x)$ is written as $m(s) \times h_{s}(x)$ where $m(s)$ is a meromorphic function on $\Omega$ and $h_{s}(x)$ is a tempered distribution with a holomorphic parameter $s \in \Omega$. We say that $u_{s}(x)$ has a pole at $s=s_{0}$ if $m(s)$ has a pole at $s_{0}$ and $u_{s}(x)$ is not a tempered distribution with a holomorphic parameter at $s=s_{0}$.

Then we have the following propositions.

Proposition 3.2. Let $\Omega$ be a domain in $\mathbb{C}$ and let $\mathbb{X}$ be a finite dimensional real vector space.

1) Let $u_{s}(x)$ and $v_{s}(x)$ be two tempered distributions on $\mathbb{X}$ with a meromorphic parameter $s \in \Omega$. If $u_{s}(x)=v_{s}(x)$ for any $s$ in an open subset $\boldsymbol{\Omega}^{\prime} \subset \boldsymbol{\Omega}$, then $u_{s}(x)=v_{s}(x)$ for any $s \in \boldsymbol{\Omega}$.

2) Let $u_{s}(x)$ be a tempered distribution on $\mathbb{X}$ with a meromorphic parameter $s \in \Omega$. Then the Fourier transform $\hat{u}_{s}\left(x^{*}\right)$ with respect to the variable $x \in \mathbb{X}$ is a tempered distribution on $\mathbb{X}^{*}$ (the dual vector space to $\mathbb{X}$ ) with a meromorphic parameter $s \in \Omega$ whose poles are located at the same place as $u_{\mathrm{s}}(x)$.

3) Let $u_{s}(x)$ be a tempered distribution on $\mathbb{X}$ with a meromorphic parameter $s \in \Omega$ and let $P\left(s, x, D_{x}\right)$ be a differential operator on $\mathbb{X}$ whose coefficients are polynomials with respect to $x$ and holomorphic with respect to s. Then $P\left(s, x, D_{x}\right) u_{s}(x)$ is a tempered distribution on $\mathbb{X}$ with a meromorphic parameter $s \in \Omega$ and the set of the locations of the poles of $P\left(s, x, D_{x}\right)$ is contained in the set of the locations of the poles of $u_{s}(x)$. 
Proof. 1) It is trivial from the uniqueness of the analytic continuation.

2) We denote by $\hat{u}(y)$ the Fourier transform of a tempered distribution $u(x)$. First, we suppose that $u_{s}(x)$ is a tempered distribution with a holomorphic parameter $s \in \Omega$. We have to show that

$$
f \longmapsto \hat{T}_{s}(f)=\int \hat{u}_{s}(y) f(y) d y \quad\left(f \in \mathscr{S}\left(\boldsymbol{X}^{*}\right)\right),
$$

defines a tempered distribution for any $s \in \Omega$, and that $T_{s}(f)$ is holomorphic with respect to $s \in \mathscr{\Omega}$ for any $f \in \mathscr{S}\left(\mathbb{X}^{*}\right)$. In fact, $\hat{T}_{s}(f)=T_{s}(\hat{f})$ by definition and $f \longrightarrow \hat{f}$ is a linear continuous isomorphism from $\mathscr{S}\left(\boldsymbol{X}^{*}\right)$ to $\mathscr{S}(\boldsymbol{X})$. Therefore,

$$
f \longmapsto \hat{T}_{s}(f)=T_{s}(\hat{f}),
$$

defines a tempered distribution on $X^{*}$ and $T_{s}(\hat{f})$ is holomorphic with respect to $s \in \mathscr{Q}$ for any $f \in \mathscr{S}\left(\boldsymbol{X}^{*}\right)$. Thus $\hat{u}_{s}(y)$ is a tempered distribution with a holomorphic parameter $s \in \boldsymbol{\Omega}$.

Next, consider the case of $u_{s}(x)$ with a meromorphic parameter $s \in \boldsymbol{\Omega}$. From the definition, $u_{s}(x)=m(s) \times v_{s}(x)$ with $m(s)$ a meromorphic function on $\boldsymbol{Q}$ and $v_{s}(x)$ a tempered distribution on $\boldsymbol{X}$ with a holomorphic parameter $s \in \Omega$. The Fourier transform is $\hat{u}_{s}(y)=m(s)$ $\times \hat{v}_{s}(y)$ and hence $\hat{u}_{s}(y)$ is a tempered distribution with a meromorphic parameter $s \in \Omega$ whose poles are located at the same place as $u_{s}(x)$. In fact, if $\hat{u}_{s}(y)$ does not have a pole at $s=s_{0}$, then $u_{s}(x)$ does not have a pole at $s=s_{0}$, i. e., holomorphic, because it is the inverse Fourier transform of $\hat{u}_{s}(y)$.

3) First, we suppose that $u_{s}(x)$ is a tempered distribution with a holomorphic parameter $s \in \Omega$. Let

$$
P\left(s, x, D_{x}\right)=\sum_{\alpha} a_{\alpha}(s, x) D_{x}^{\alpha}, \quad(\text { a finite sum), }
$$

where $a_{\alpha}(s, x)$ is a polynomial whose coefficients are holomorphic functions in $s \in \Omega$. Namely, we have

$$
a_{\alpha}(s, x)=\sum_{\beta} a_{\alpha \beta}(s) x^{\beta}, \quad \text { (a finite sum), }
$$

with $a_{\alpha \beta}(s)$ a holomorphic function in $s \in \Omega$. Therefore we have to show that $v_{\alpha \beta s}(x)=a_{\alpha \beta}(s) x^{\beta} D_{x}^{\alpha} u_{s}(x)$ is a tempered distribution with a holomorphic parameter $s \in \mathbb{R}$. Apparently, $v_{\alpha \beta s}(x)$ is a tempered distribution for any fixed $s \in \Omega$. Consider the integral 


$$
\begin{aligned}
T_{\alpha \beta s}(x) & =\int v_{\alpha \beta s}(x) f(x) d x=\int a_{\alpha \beta}(s)\left(x^{\beta} D_{x}^{\alpha} u_{s}(x)\right) f(x) d x \\
& =(-1)^{|\alpha|} a_{\alpha \beta}(s) \int u_{s}(x)\left(D_{x}^{\alpha}\left(x^{\beta} f(x)\right)\right) d x .
\end{aligned}
$$

Here, $f(x) \in \mathscr{S}(\mathbb{X})$. Then $D_{x}^{\alpha}\left(x^{\beta} f(x)\right) \in \mathscr{S}(\mathbb{X})$ and hence $T_{\alpha \beta s}(x)$ is holomorphic in $s \in \Omega$. Thus $v_{\alpha \beta s}(x)$ is a tempered distribution with a holomorphic parameter $s \in \mathbb{R}$.

Next, consider the case that $u_{s}(x)$ is a tempered distribution with a meromorphic parameter $s \in \mathbb{R}$. However, it is evident from the definition that $P\left(s, x, D_{x}\right) u_{s}(x)$ is a tempered distribution with a meromorphic parameter $s \in \mathbb{\Omega}$, because

$$
\begin{aligned}
P\left(s, x, D_{x}\right) u_{s}(x) & =P\left(s, x, D_{x}\right)\left(m(s) \times v_{s}(x)\right) \\
& =m(s) \times P\left(s, x, D_{x}\right) v_{s}(x),
\end{aligned}
$$

where $m(s)$ is a meromorphic function on $\mathscr{R}$ and $v_{s}(x)$ is a tempered distribution with a holomorphic parameter $s \in 9$. The locations of the poles of $P\left(s, x, D_{x}\right) u_{s}(x)$ is continued in those of $m(s)$ and we have the result.

q.e.d.

Let us consider some examples of tempered distributions with a meromorphic parameter. Recall the connected component decomposition of $\mathbb{V}_{R}-S_{R}$ in the preceding section;

$$
\mathbb{V}_{n}^{(n)} \cup \mathbb{V}_{n-1}^{(n)} \cup \ldots \cup \mathbb{V}_{0}^{(n)}=\mathbb{V}_{R}-\mathbb{S}_{R}
$$

where $\mathbb{V}_{i}^{(n)}$ is the connected component of $\mathbb{V}_{R}-\mathbb{S}_{R}$ consisting of the elements of signature $(i, n-i)$. We define the hyperfunction,

$$
|P|_{i}^{s}(x)= \begin{cases}|P(x)|^{s} & \text { if } x \in \mathbb{V}_{i}^{(n)}, \\ 0 & \text { if } x \notin \mathbb{V}_{i}^{(n)} .\end{cases}
$$

This hyperfunction $|P|{ }_{i}^{s}(x)$ is a continuous function when $s$ has a sufficiently large real part and is a tempered distribution on $\mathbb{V}_{\boldsymbol{R}}$ with a holomorphic parameter $s \in \mathbb{\Omega}_{k}=\{s \in \mathbb{C} ; \operatorname{Re}(s)>k\}$. Here we put $k$ to be sufficiently large so as that $|P|_{i}^{s}(x)$ is a continuous function on $\mathbb{V}_{\boldsymbol{R}}$. In fact, if $s \in \mathbb{\Omega}_{k}$, then

$$
\phi \longmapsto \int|P|_{i}^{s}(x) \phi(x) d x \quad\left(\phi \in \mathscr{S}\left(\mathbb{V}_{R}\right)\right)
$$

is convergent and defines a tempered distribution on $\mathbb{V}_{\boldsymbol{R}}$. We shall continue $|P|_{i}^{s}(x)$ as a tempered distribution with a meromorphic 
parameter $s \in C$ in the following way. When $s \in \Omega_{k}$, the tempered distribution $|P|_{i}^{s}(x)$ is a continuous function on $V$. By Proposition 1.4, we have, modulo constant multiples,

$$
P\left(D_{x}\right)|P(x)|_{i}^{s+1}=b(s)|P(x)|_{i}^{s},
$$

where $b(s)$ is the $b$-function of $P^{s}(x)$. When $\phi(x) \in \mathscr{S}(\boldsymbol{V})$, we have

$$
\begin{gathered}
\int|P|_{i}^{s}(x) \phi(x) d x=\int b(s)^{-1}\left(P\left(D_{x}\right)|P|_{i}^{s+1}(x)\right) \phi(x) d x \\
=b(s)^{-1} \int|P|_{i}^{s+1}(x)\left(P\left(-D_{x}\right) \phi(x)\right) d x,
\end{gathered}
$$

and hence $|P(x)|_{i}^{s}$ is defined by this formula for $s \in \Omega_{k-1}$. We can define $|P|_{i}^{s}(x)$ for $s \in \boldsymbol{\Omega}_{k-m}$ inductively by

$$
\int|P|_{i}^{s}(x) \phi(x) d x=\prod_{j=0}^{m-1} b(s+j)^{-1} \int|P|_{i}^{s+m}(x)\left(P\left(-D_{x}\right)^{m} \phi(x)\right) d x .
$$

Thus $|P|_{i}^{s}(x)$ is well defined as a tempered distribution with a meromorphic parameter $s \in C$. The poles of $|P|_{i}^{s}(x)$ with respect to $s$ are located in the set,

$$
\{s \in C ; s \text { is a root of } b(s+j)=0 \text { with some } j=0,1,2, \ldots\} .
$$

Definition 3.3 (critical points). We say that $s \in C$ is a critical point for $P(x)^{s}$ if

$$
s \in\left\{s_{i}-j \in C ; s_{i} \text { is a root of } b(s)=0 \text { and } j=1,2,3, \ldots\right\},
$$

and we denote by $\operatorname{Crit}\left(P(x)^{s}\right)$ the set of critical points for $P(x)^{s}$. The hyperfunction $|P|_{i}^{s}(x)$ is well defined for any $s \notin \operatorname{Crit}\left(P(x)^{s}\right)$ and has a possible poles at the points in $\operatorname{Crit}\left(P(x)^{s}\right)$.

Proposition 3.4. 1) Let $s$ be a point in the complement of $\operatorname{Crit}\left(P(x)^{s}\right)$. Then the hyperfunction $|P|_{i}^{s}(x)$ is a solution to the holonomic system $\mathfrak{M}_{s}$, i. e., $\left(\left\langle d \rho(A) x, D_{x}\right\rangle-s \delta \chi(A)\right)|P|_{i}^{s}(x)=0$ for any $A \in \mathscr{G}_{\boldsymbol{R}}$.

2) Let $\Gamma_{q}(s)=\prod_{i=1}^{q} \Gamma\left(s-s_{i}\right)$. Here $s_{i}$ is the roots of the $b$-function, which is defined in (2.50) explicitly. Then $\Gamma_{q}(s)^{-1}|p|_{j}^{s}(x)(j=0,1, \ldots, n)$ is a tempered distribution with a holomorphic parameter $s$ in the domain $\left\{s \in \boldsymbol{C} ; \operatorname{Re}(s)>s_{q+1}\right\}$. In particular, when $q=n, \Gamma_{n}(s)^{-1}|P|_{j}^{s}(x)$ is a tempered distribution with a holomorphic parameter $s$ in $\mathbb{C}$. Moreover, if $\operatorname{Re}(s)>s_{q+1}(r e s p . s \in C)$, then $\Gamma_{q}(s)^{-1}|P|_{j}^{s}(x)\left(\operatorname{resp} . \Gamma_{n}(s)^{-1}|P|_{j}^{s}(x)\right)$ is a solution to the holonomic system $\mathfrak{M}_{s}$. 
Proof. 1) Let $A \in \mathscr{G}_{\boldsymbol{R}}$. Then $\exp (t A) \in \mathbb{G}_{\boldsymbol{R}}^{+}$for sufficiently small $t \in \mathbb{R}$. We have

$$
|P|_{i}^{s}\left(\rho(\exp (t A) x)=\chi(\exp (t A))|P|_{i}^{s}(x),\right.
$$

for any $s \in \mathcal{C}$ with a sufficiently large real part since $|P|_{i}^{s}(x)$ is a continuous function on $V_{R}$. By differentiating (3.7) with $t$ and by putting $t=0$, we have

$$
\left\langle d \rho(A) x, D_{x}\right\rangle|P|_{i}^{s}(x)=s \delta \chi(A)|P|_{i}^{s}(x),
$$

for $s \in C$ with a sufficiently large real part so as that $|P|_{i}^{s}(x)$ is $C^{1-}$ class. Thus, $|P|_{i}^{s}(x)$ is a solution of $\mathfrak{M}_{\mathrm{s}}$ for any $s \in \mathbb{C}$ with a sufficiently large real part. Since $|P|_{i}^{s}(x)$ is continued to the complex plane as a tempered distribution with a meromorphic parameter $s \in \mathbb{C}$, both hands of (3.8) are tempered distributions with a meromorphic parameter $s \in C$ by Proposition 3.2. The equation (3.8) holds for any $s \in C$-Crit $\left(P(x)^{s}\right)$ by the analytic continuation.

2) Note that,

$$
b(s) b(s+1) \ldots b(s+m) \cdot|P|_{i}^{s}(x),
$$

is a tempered distribution with a holomorphic parameter $s$ in $\boldsymbol{\Omega}_{-m-1}$ $=\{s \in \mathbb{C} ; \operatorname{Re}(s)\rangle-m-1\}$. In fact, if $|P|_{i}^{s}(x)$ is holomorphic with respect to $s$ in $\Omega_{k}$, then (3.9) is holomorphic with respect to $s$ in $\boldsymbol{\Omega}_{k-m}$. We take $m$ a sufficiently large integer. All the zeros of $b(s), b(s+1), \ldots, b(s+m)$ are contained in $\operatorname{Re}(s) \leqq-1$, hence $|P|_{i}^{s}(x)$ actually does not have a pole in $\operatorname{Re}(s)>-1$, i. e., holomorphic. Similarly, all the zeros of $b(s+1), b(s+2), \ldots, b(s+m)$ are contained in $R(s) \leqq-2$, and hence $b(s)|P|_{i}^{s}(x)$ does not have a pole in $\operatorname{Re}(s) \geqq-2$. Moreover, since all the zeros of $b(s+k), b(s+k+2)$, $\ldots, b(s+m)$ are contained in $\operatorname{Re}(s) \leqq-(k+1)$,

$$
b(s) b(s+1) \ldots b(s+k-1)|P|_{i}^{s}(x),
$$

is holomorphic in $\operatorname{Re}(s)>-(k+1)$.

Next we consider $\Gamma_{q}(s)^{-1}|P|_{i}^{s}(x) . \Gamma_{1}(s)^{-1}=\Gamma\left(s-s_{1}\right)^{-1}$ has a simple pole at $s=s_{1}-k(k=0,1,2, \ldots)$. Namely, among the poles of

$$
b(s+k)^{-1}=\left(s-s_{1}+k\right)^{-1}\left(s-s_{2}+k\right)^{-1} \ldots\left(s-s_{n}+k\right)^{-1},
$$

the first pole $\left(s-s_{1}+k\right)^{-1}$ is canceled by $\Gamma\left(s-s_{1}\right)^{-1}$. Therefore, for any integer $m \geqq 0$, the poles of

$$
\Gamma_{1}(s)^{-1} b(s)^{-1} b(s+1)^{-1} \ldots b(s+m)^{-1},
$$


are located in $\operatorname{Re}(s) \leqq s_{2}$ since $s_{1}>s_{2}>s_{3} \ldots>s_{n}$. Similarly, among the poles of (3.11), the poles $\left(s-s_{1}+k\right)^{-1},\left(s-s_{2}+k\right)^{-1}, \ldots,\left(s-s_{q}+k\right)^{-1}$ are canceled by $\Gamma_{q}(s)^{-1}=\Gamma\left(s-s_{1}\right)^{-1} \Gamma\left(s-s_{2}\right)^{-1} \ldots \Gamma\left(s-s_{q}\right)^{-1}$. Then, for any integer $m \geqq 0$, the poles of

$$
\Gamma_{q}(s)^{-1} b(s)^{-1} b(s+1)^{-1} \ldots b(s+m)^{-1}
$$

is located in $\operatorname{Re}(s) \leqq s_{q+1}$. In particular, if $q=n$, then all the poles of (3.11) are canceled by $\Gamma_{n}(s)^{-1}$, and hence $\Gamma_{n}(s)^{-1} b(s)^{-1} b(s+1)^{-1} \ldots$ $b(s+m)^{-1}$ is an entire function for any integer $m \geqq 0$. Therefore,

$$
\Gamma_{q}(s)^{-1}|P|_{i}^{s}(x)=\Gamma_{q}(s)^{-1} \prod_{j=0}^{m} b(s+j)^{-1}\left(\prod_{j=0}^{m} b(s+j)\right)|P|_{i}^{s}(x),
$$

is a tempered distribution with a holomorphic parameter $s$ in $\{s \in \boldsymbol{C}$; $\left.\operatorname{Re}(s)>\max \left\{-m-1, s_{q+1}\right\}\right\}$, for any integer $m \geqq 0$, and hence it is holomorphic in $\operatorname{Re}(s)>s_{q+1}$. In particular," if $q=n$, then (3.13) is holomorphic in $\boldsymbol{\Omega}_{-m-1}$ for any integer $m \geqq 0$, and hence it is entire with respect to $s$.

Lastly, we shall show that $\Gamma_{q}(s)^{-1}|P|_{i}^{s}(x)$ is a solution to $\mathfrak{M}_{s}$ for any $s$ in $\operatorname{Re}(s)>s_{q+1}$. In fact, since

$$
\left(\left\langle d \rho(A) x, D_{x}\right\rangle-s \delta \chi(A)\right) \Gamma_{q}(s)^{-1}|P|_{i}^{s}(x)=0,
$$

for any $s \in \boldsymbol{C}$ with a sufficiently large real part, we have the result by an analytic continuation to $\left\{s \in \boldsymbol{C} ; \operatorname{Re}(s)>s_{q+1}\right\}$ (Proposition 3.2,1)). In particular, if $q=n$, then (3.14) is valid for any $s \in C$.

q.e.d.

We introduce the Euclidean measure $d x$ on $V_{\boldsymbol{R}}$ by

$$
\begin{aligned}
& \text { 1) } d x=\left|\left(\bigwedge_{i=1}^{n} d x_{i i}\right) \wedge\left(\bigwedge_{i<j} d x_{i j}\right)\right| \text {, when } V_{\boldsymbol{R}}=\boldsymbol{S} \boldsymbol{y} \boldsymbol{m}(n, \boldsymbol{R}) . \\
& \text { 2) } d x=\mid\left(\bigwedge_{i=1}^{n} d x_{i i}\right)\left(\bigwedge_{i<j}\left(d \operatorname{Re}\left(x_{i j}\right) \wedge d \operatorname{Im}\left(x_{i j}\right)\right) \mid\right. \text {, when } \\
& \boldsymbol{V}_{\boldsymbol{R}}=\boldsymbol{H e r}(n, \boldsymbol{C}) . \\
& \text { 3) } d x=\left|\left(\bigwedge_{i=1}^{n} d x_{i i}\right)\left(\bigwedge_{i<j}\left(d x_{i j}^{1} \wedge d x_{i j}^{2} \wedge d x_{i j}^{3} \wedge d x_{i j}^{4}\right)\right)\right| \text {, when } \\
& \boldsymbol{V}_{\boldsymbol{R}}=\boldsymbol{H e r}(n, \boldsymbol{H}) . \text { Here, we write } x_{i j}=x_{i j}^{1}+x_{i j}^{2} e_{1}+x_{i j}^{3} e_{2} \\
& +x_{i j}^{4} e_{1} e_{2} \text { with } x_{i j}^{k} \in \boldsymbol{R} \text { and } e_{1}^{2}=e_{2}^{2}=-1, e_{1} e_{2}=-e_{2} e_{1} .
\end{aligned}
$$

We define the Fourier transform of $u(x) \in \mathscr{S}\left(V_{R}\right)$ by

$$
\hat{u}(y)=\int u(x) \exp (2 \pi \sqrt{-1}\langle x, y\rangle) d x,
$$


and the inverse Fourier transform for $u(y) \in \mathscr{S}\left(\mathbb{V}_{R}^{*}\right)$, by

$$
\check{u}(x)=\int u(y) \exp (-2 \pi \sqrt{-1}\langle x, y\rangle) d y \text {. }
$$

Thus, as a well known result, we have

$$
\widehat{\hat{u}}(x)=2^{-n(n-1) v / 2} u(x) \text { and } \widehat{\widehat{u}}(y)=2^{-n(n-1) v / 2} u(y),
$$

where $v=1 \quad\left(\mathbb{V}_{\boldsymbol{R}}=\operatorname{Sym}(n, \mathbb{R})\right), 2\left(\mathbb{V}_{\boldsymbol{R}}=\mathbb{H} \boldsymbol{H e r}^{r}(n, \mathbb{C})\right)$ and $4 \quad\left(\mathbb{V}_{\boldsymbol{R}}=\mathbb{H}\right.$ er $(n, \mathbb{H}))$.

Recall the holonomic system

$$
\mathfrak{M}_{s} ;\left(\left\langle d \rho(A) x, D_{x}\right\rangle-s \delta \chi(A)\right) u(x)=0 .
$$

Here, $d \rho$ and $\delta \chi$ are the infinitesimal representation of $\rho$ and the infinitesimal character of $\chi ;\langle$,$\rangle is the bilinear form on \mathbb{V}_{\boldsymbol{R}} \times \mathbb{V}_{\boldsymbol{R}}^{*}$ defined in (2.4); $s$ is a complex number. We define the "dual" holonomic system on $\mathbb{V}_{\boldsymbol{R}}^{*}$ of $\mathfrak{M}_{s}$ by

$$
\mathfrak{M}_{s}^{*} ;\left(\left\langle d \rho^{*}(A) y, D_{y}\right\rangle-s \delta \chi(A)\right) v(y)=0 \text { 。 }
$$

Here, $d \rho^{*}$ is the infinitesimal representation of the contragredient representation of $\rho$. Then we have the following propositions.

Proposition 3.5. 1) If we identify $\mathbb{V}_{\boldsymbol{R}}$ and $\mathbb{V}_{\boldsymbol{R}}^{*}$ by the inner product $\langle x, y\rangle$ defined in (2.4), then the holonomic systems $\mathfrak{M}_{s}$ and $\mathfrak{M}_{-s}^{*}$ are the same.

2) We denote by $\mathrm{Sol}\left(\mathfrak{M}_{s}\right)_{t e m}$ the tempered distribution solution space to the holonomic system $\mathfrak{M}_{s}$. Then we have

(1) $u(x) \in \operatorname{Sol}\left(\mathfrak{M}_{s}\right)_{t e m} \Leftrightarrow \hat{u}(y) \in \operatorname{Sol}\left(\mathfrak{M}_{s+\left(n^{\prime} / n\right)}^{*}\right)_{t e m}$ 。

(2) $v(y) \in \operatorname{Sol}\left(\mathfrak{M}_{s}^{*}\right)_{t e m} \Leftrightarrow \check{v}(x) \in \operatorname{Sol}\left(\mathfrak{M}_{s-\left(n^{\prime} / n\right)}\right)_{t e m}$.

(1) $u(x) \in \operatorname{Sol}\left(\mathfrak{M}_{s}\right)_{t e m} \Leftrightarrow u(x)$ is a tempered distribution on $\mathbb{V}_{\boldsymbol{R}}$ and $u(\rho(g) x)=\chi(g)^{s} u(x)$ for all $g \in \mathbb{G}_{R^{+}}$.

(2) $v(y) \in \operatorname{Sol}\left(\mathfrak{M}_{s}^{*}\right)_{t e m} \Leftrightarrow v(y)$ is a tempered distribution on $\mathbb{V}_{R}^{*}$ and $v\left(\rho^{*}(g) y\right)=\chi(g)^{s} v(y)$ for all $g \in G_{R}^{+}$.

3) Let $u(x) \in \operatorname{Sol}\left(\mathfrak{M}_{s}\right)_{t e m}\left(\operatorname{resp} . \quad v(y) \in \operatorname{Sol}\left(\mathfrak{M}_{s+\left(n^{\prime} / n\right)}^{*}\right)_{t e m}\right)$. Then $u(x)$ (resp. $v(y)$ ) is real analytic on $\mathbb{V}_{R}-\mathbb{S}_{R}\left(\operatorname{resp} . \mathbb{V}_{R}^{*}-\mathbb{S}_{R}^{*}\right)$ and
(1) $\left.u(x)\right|_{V_{R}-S_{R}}=\left.\sum_{i} a_{i}|P|_{i}^{s}(x)\right|_{V_{R}-s_{R}}$
(2) $\left.v(y)\right|_{V_{R}^{*}-S_{R}^{*}}=\left.\sum_{i} b_{i}|P|_{i}^{-s-\left(n^{\prime} / n\right)}(y)\right|_{V_{R}^{*}-S_{R}^{*}}$ 
with some constants $a_{i} \in C\left(\right.$ resp. $\left.b_{i} \in \boldsymbol{C}\right)$.

Proof. 1) Note that we identify $\boldsymbol{V}_{\boldsymbol{R}}$ and $\boldsymbol{V}_{\boldsymbol{R}}^{*}$ by the inner product $\langle x, y\rangle=\operatorname{tr}\left(x^{t} \tilde{y}\right)$. Then the Lie algebra $\mathscr{G}_{\boldsymbol{R}}$ is naturally identified with a Lie subalgebra in $\mathfrak{g} \mathfrak{l}\left(\mathbb{V}_{\boldsymbol{R}}\right)=\mathfrak{g} \mathfrak{l}\left(\mathbb{V}_{\boldsymbol{R}}^{*}\right)$ by $d \rho$ and $d \rho^{*}$. However, the images of $\mathscr{G}_{R}$ by $d \rho$ and $d \rho^{*}$ coincides with each other by the automorphism $\phi ; A \rightarrow-{ }^{t} \bar{A}$. In fact, we have

$$
\begin{aligned}
& d \rho(A) x=A x+x^{t} A, d \rho^{*}(A) x=-{ }^{t} \bar{A} x-x \bar{A}, \\
& \delta \chi(A)=-\delta \chi\left(-{ }^{t} \bar{A}\right)=\delta \chi\left({ }^{t} \bar{A}\right) .
\end{aligned}
$$

Therefore, we have

$$
\begin{aligned}
& \left\{\left\langle d \rho(A) x, D_{x}\right\rangle-s \delta \chi(A) ; A \in \mathscr{G}_{R}\right\} \\
& =\left\{\left\langle d \rho^{*}(A) x, D_{x}\right\rangle+s \delta \chi\left({ }^{t} \bar{A}\right) ; A \in \mathscr{G}_{R}\right\} .
\end{aligned}
$$

Thus we have the result.

2) First we shall show that (3.21) (1). Then (3.21) (2) is evident by 1). Let $u(x) \in \operatorname{Sol}\left(\mathfrak{M}_{s}\right)_{t e m}$. Then, for any $f(x) \in \mathscr{S}\left(\boldsymbol{V}_{\boldsymbol{R}}\right)$, we have

$$
\int u(x)\left\langle d \rho(A) x, D_{x}\right\rangle^{*} f(x) d x=s \delta \chi(A) \int u(x) f(x) d x .
$$

Here, $\left\langle d \rho(A) x, D_{x}\right\rangle^{*}$ stands for the formal adjoint operator of $\langle d \rho(A) x$, $\left.D_{x}\right\rangle$. Since

$$
\begin{aligned}
\left\langle d \rho(A) x, D_{x}\right\rangle^{*} & =-\left\langle D_{x}, d \rho(A) x\right\rangle \\
& =-\left\langle d \rho(A) x, D_{x}\right\rangle-\operatorname{tr}(d \rho(A)) \\
& =-\left\langle d \rho(A) x, D_{x}\right\rangle-\left(n^{\prime} / n\right) \delta \chi(A),
\end{aligned}
$$

we have

$$
\int u(x)\left(-\left\langle d \rho(A) x, D_{x}\right\rangle\right) f(x) d x=\left(s+\left(n^{\prime} / n\right)\right) \delta \chi(A) \int u(x) f(x) d x .
$$

On the other hand, for a sufficiently small $t \in \boldsymbol{R}$, the element $g=\exp (t A) \in \boldsymbol{G}_{\boldsymbol{R}}^{+}$is defined and

$$
\begin{aligned}
& \sum_{m=0}^{\infty}\left(t^{m} / m !\right)\left(-\left\langle d \rho(A) x, D_{x}\right\rangle\right)^{m} f(x)=f\left(\rho(g)^{-1} x\right), \\
& \sum_{m=0}^{\infty}\left(t^{m} / m !\right)\left(s+\left(n^{\prime} / n\right)\right)^{m} \delta \chi(A)^{m} f(x)=\chi(g)^{s+\left(n^{\prime} / n\right)} f(x),
\end{aligned}
$$

are convergent in $\mathscr{S}\left(\mathbb{V}_{R}\right)$. Thus we have

$$
\int u(x) f\left(\rho(g)^{-1} x\right) d x
$$




$$
\begin{aligned}
& =\int u(x) \sum_{m=0}^{\infty}\left(t^{m} / m !\right)\left(-\left\langle d \rho(A) x, D_{x}\right\rangle\right)^{m} f(x) d x \\
& =\int u(x) \sum_{m=0}^{\infty}\left(t^{m} / m !\right)\left(s+\left(n^{\prime} / n\right)\right)^{m} \delta \chi(A)^{m} f(x) d x \\
& =\int u(x) \chi(g)^{s+\left(n^{\prime} / n\right)} f(x) d x .
\end{aligned}
$$

Since

$$
\begin{aligned}
& \int u(x) f\left(\rho(g)^{-1} x\right) d x \\
& =\int u(\rho(g) x) f(x) d(\rho(g) x)=\chi(g)^{\left(n^{\prime} / n\right)} \int u(\rho(g) x) f(x) d x
\end{aligned}
$$

we have

(3.29) $\int u(\rho(g) x) f(x) d x=\chi(g)^{s} \int u(x) f(x) d x$,

for all $f(x) \in \mathscr{S}\left(\boldsymbol{V}_{\boldsymbol{R}}\right)$. Thus we have

$$
u(\rho(g) x)=\chi(g)^{s} u(x),
$$

with $g=\exp (i A)$ for sufficiently small $t \in \mathbb{R}$. Any element $g \in \mathbb{G}_{\boldsymbol{R}}^{+}$is written as

$$
g=\exp \left(t_{1} A_{1}\right) \ldots \exp \left(t_{k} A_{k}\right),
$$

with $t_{i} \in \mathbb{R}$ and $A_{i} \in \mathscr{G}_{\boldsymbol{R}}$. Therefore (3.30) is valid for all $g \in \mathbb{G}_{\boldsymbol{R}}^{+}$.

Conversely, if (3.29) is valid, then (3.28) is also true. By differentiating (3.28) by $t$ and by putting $t=0$, we have (3.26). Thus, we have $u(x) \in \operatorname{Sol}\left(\mathfrak{M}_{s}\right)_{t e m}$.

Next we shall show $(3.20),(1)$. We have to show that

$$
u(\rho(g) x)=\chi(g)^{s} u(x) \Leftrightarrow \hat{u}\left(\rho^{*}(g) y\right)=\chi(g)^{s+\left(n^{\prime} / n\right)} \hat{u}(y) .
$$

We suppose that $u(\rho(g) x)=\chi(g)^{s} u(x)$. Then we have

$$
\begin{aligned}
& \int \hat{u}\left(\rho^{*}(g) y\right) f(y) d y=\int \hat{u}(y) f\left(\rho^{*}(g)^{-1} y\right) d\left(\rho^{*}(g)^{-1} y\right) \\
& \left.\quad=\chi(g)^{\left(n^{\prime} / n\right)} \int u(x) \widehat{f\left(\rho^{*}(g)^{-1}\right.} y\right) d x \\
& \quad=\int u(x) \hat{f}\left(\rho(g)^{-1} x\right) d x \\
& \quad=\int u(\rho(g) x) \hat{f}(x) d(\rho(g) x)=\chi(g)^{s+\left(n^{\prime} / n\right)} \int u(x) \hat{f}(x) d x
\end{aligned}
$$

because, 


$$
\begin{aligned}
& \left.\widehat{f\left(\rho^{*}(g)^{-1}\right.} y\right)=\int f\left(\rho^{*}(g)^{-1} y\right) \exp (2 \pi \sqrt{-1}\langle x, y\rangle) d y \\
& \quad=\int f(y) \exp \left(2 \pi \sqrt{-1}\left\langle x, \rho^{*}(g) y\right\rangle\right) d\left(\rho^{*}(g) y\right) \\
& =\chi(g)^{-\left(n^{\prime} / n\right)} \int f(y) \exp \left(2 \pi \sqrt{-1}\left\langle\rho(g)^{-1} x, y\right\rangle\right) d y \\
& =\chi(g)^{-\left(n^{\prime} / n\right)} \hat{f}\left(\rho(g)^{-1} x\right) .
\end{aligned}
$$

Thus we have

$$
\hat{u}\left(\rho^{*}(g) y\right)=\chi(g)^{s+\left(n^{\prime} / n\right)} \hat{u}(y) .
$$

The converse is shown in the same way. For (3.20), (2), the proof is the same.

3) In fact, $\mathfrak{M}_{s}$ is an elliptic system on $\mathbb{V}_{R}-\mathbb{S}_{R}$, and hence $u(x)$ is real analytic on $\mathbb{V}_{R}-\mathbb{S}_{R}$. Moreover, since $\mathfrak{M}_{s}$ is a simple holonomic system on $\mathbb{V}_{\boldsymbol{R}}-\mathbb{S}_{\boldsymbol{R}}$ and $|P|_{i}^{s}(x)$ is a basis of the solution on $\mathbb{V}_{i}^{(n)}$, we have $(3.22),(1)$. For (3.22), (2), we can show it in the same way.

q. e. d.

Proposition 3. 6. Let $s \notin \operatorname{Crit}\left(P(x)^{s}\right)$. Then we have

$$
\begin{aligned}
\sigma_{V_{j}^{(n)} \times\{0\}}\left(|P|_{i}^{s}(x)\right) & = \begin{cases}|P(x)|^{s} & \text { if } i=j, \\
0 & \text { if } i \neq j .\end{cases} \\
\sigma_{\{0\} \times V_{j}^{*(n)}}\left(|P|_{i}^{s}(x)\right) & =(2 \pi)^{n s+\left(n^{\prime} / 2\right)} 2^{n(n-1) / 2} \\
& \times|P|_{i}^{s}(y) \sqrt{|d y|} /\left.\sqrt{|d x|}\right|_{V_{j}^{*}}
\end{aligned}
$$

Here, $\{0\} \times \mathbb{V}_{j}^{*(n)}\left(\operatorname{resp} \cdot \mathbb{V}_{i}^{(n)} \times\{0\}\right)$ is the Lagrangian subvariety $\Lambda_{n}^{0 i}$ (resp. $\left.\Lambda_{0}^{i 0}\right)$.

Proof. (3.34) is evident by definition, because $|P|_{i}^{s}(x)$ is a real analytic function on $\mathbb{V}_{j}^{(n)}$ (Definition 2.7)。

Now we consider the tempered distribution

$$
|P|_{i}^{s}(x)=\int|P|_{i}^{s}(y) \exp (2 \pi \sqrt{-1}\langle x, y\rangle) d y\left(2^{n(n-1) v / 2}\right)
$$

The distribution $|P|_{i}^{s}(y)$ is a homogeneous distribution on $\mathbb{V}_{\boldsymbol{R}}^{*}$ of degree $-s n-n^{\prime}$. In fact, it is proved by that $|P|_{i}^{s}(x)$ is a homogeneous distribution on $\mathbb{V}_{\boldsymbol{R}}$ of degree $s n$. Therefore, we can view $\left.|P|_{i}^{s}(y)\right|_{V_{R}^{*}-\{0\}}$ as a distribution $|\stackrel{V}{P}|_{i}^{s}(r \omega)=r^{-n_{s}-n}|\stackrel{V}{P}|_{i}^{s}(\omega)$ on $(r, \omega) \in$ $R_{+} \times S^{n^{\prime}-1}$ where $S^{n^{\prime}-1}$ is the unit sphere in $\mathbb{V}_{\boldsymbol{R}}^{*}$ by the map 


$$
\begin{gathered}
(r, \omega) \longmapsto r \omega \\
\prod \\
\mathbb{R}_{+} \times S^{n^{\prime}-1} \rightarrow \mathbb{V}_{R}^{*}-\{0\}
\end{gathered}
$$

Moreover, we can extend this as a distribution on $R \times S^{n^{\prime-1}}$ by

$$
|P|_{i}^{s}(r \omega)=|P|_{i}^{s}(\omega) r_{+}^{-n s-n^{\prime}}
$$

where $r_{+}^{-n^{s-n^{\prime}}}$ is a tempered distribution that is equal to $r^{-n s-n^{\prime}}$ on $r>0$ and is equal to 0 on $r<0$. (Of course such distribution is not uniquely determined.) The distribution (3.38) is naturally a tempered distribution on $\mathbb{V}_{\boldsymbol{R}}^{*}$ by

$$
f(x) \longmapsto \int_{-\infty}^{+\infty} \int_{S^{n^{\prime}-1}}|P|_{i}^{\vee}(\omega) r_{+}^{-n s-n} f(r \omega) r^{n^{\prime}-1} d r d \omega
$$

with $f(x) \in \mathscr{S}\left(\mathbb{V}_{R}^{*}\right)$. Here $d \omega$ is the rotation invariant measure on $S^{n^{\prime}-1}$. The tempered distribution (3.39) coincides with $|P|_{i}^{s}(y)$ except for the origin. Therefore, we have

$$
\begin{aligned}
|P|_{i}^{s}(x)= & \int_{s^{n^{\prime}-1}}|P|_{i}^{\vee}(\omega)\left(\int_{-\infty}^{+\infty} r_{+}^{-n s-n^{\prime}+n^{\prime}-1} \exp (2 \pi \sqrt{-1} r\langle x, \omega\rangle) d r\right) d \omega \\
& +O(x),
\end{aligned}
$$

where $O(x)$ is a real analytic function on $\mathbb{V}_{\boldsymbol{R}}$. In fact, the Fourier transform of any compactly supported distribution is an entire function. Note that the Fourier transform of $r_{+}^{\lambda}(\lambda \in \mathbb{C})$ is calculated to be

$$
\begin{aligned}
\int_{-\infty}^{+\infty} r_{+}^{\lambda} \exp (\sqrt{-1} r \sigma) d r= & \exp \left(\frac{\pi}{2} \sqrt{-1}(\lambda+1)\right) \Gamma(\lambda+1)(\sigma+i 0)^{-\lambda-1} \\
& +O(\sigma) \\
= & \Phi_{\lambda+1}(\sqrt{-1}(\sigma+i 0))+O(\sigma)
\end{aligned}
$$

Here $O(\sigma)$ is a real analytic function in $\sigma$ and $\Phi_{\lambda+1}(\sqrt{-1}(\sigma+i 0))$ is well defined for any $\lambda \in \mathbb{C}$ as a microfunction whose singular support is $(0, d \sigma)$ and the meaning of the expression (3.41) is a hyperfunction which coincides with $\Phi_{\lambda+1}(\sqrt{-1}(\sigma+i 0))$ modulo real analytic functions. (For the definition of $\Phi_{\lambda}$, see $\S 2$, and for the proof, for example, see Gelfand-Shilov [3].) Thus we have

$$
\text { (3.42) } \begin{aligned}
|P|_{i}^{s}(x)= & \int_{-n s}(\sqrt{-1}(2 \pi\langle x, \omega\rangle+i 0))|P|_{i}^{s}(\omega) d \omega \circ\left(2^{n(n-1) v / 2}\right) \\
& +O(x)
\end{aligned}
$$




$$
\begin{aligned}
= & (2 \pi)^{n s} \cdot 2^{n(n-1) v / 2} \int|P|_{i}^{s}(\omega) \Phi_{-n s}(\sqrt{-1}(\langle x, \omega\rangle+i 0)) d \omega \\
& +O(x) .
\end{aligned}
$$

Therefore, the microfunction $|P|_{i}^{s}(x)$ is expressed on $\{0\} \times V_{j}^{*}$ by the integral (3.42) since $|P|_{i}^{s}(\omega)$ is real analytic on $V_{j}^{*}$. Thus, from the definition of the real principal symbol, we have

$$
\begin{aligned}
& \sigma_{(0\} \times V_{j}^{*}}\left(|P|_{i}^{s}(x)\right) \\
& \quad=(2 \pi)^{\left(n^{\prime} / 2\right)+n s} 2^{n(n-1) v / 2}|P|_{i}^{s}(\omega) r^{-n s-n} \sqrt{|d y|} /\left.\sqrt{|d x|}\right|_{V_{j}^{*}} \\
& =(2 \pi)^{\left(n^{\prime} / 2\right)+n s} 2^{n(n-1) v / 2}|P|_{i}^{s}(y) \sqrt{|d y|} /\left.\sqrt{|d x|}\right|_{V_{j}^{*}} .
\end{aligned}
$$

q. e. d.

\section{Proposition 3. 7.}

$$
\left|P_{\boldsymbol{A}_{n \boldsymbol{R}}^{0 i}}\right| \sqrt[s]{\left|\omega_{\Lambda_{n \boldsymbol{R}}^{0 i}}\right|}=\left|K_{0}\right| \sqrt[s]{\left|K_{1}\right|}|P|_{i}^{-s-\frac{n^{\prime}}{n}}(y) \sqrt{|d y|},
$$

where $K_{0}=1$ and $K_{1}=2^{\frac{n(n-1) v}{2}}$.

Proof. Note that $\Lambda_{n}=\{0\} \times V^{*}$ and that

$$
\begin{aligned}
\boldsymbol{W}= & \text { Zariski closure of } \\
& \left\{(x, s \cdot \operatorname{grad} \cdot \log P(x)) \in \mathbb{T}^{*} \boldsymbol{V} ; s \in \boldsymbol{R}, \operatorname{det}(x) \neq 0\right\}, \\
= & \text { Zariski closure of } \\
& \left\{(s \cdot \operatorname{grad} \cdot \log P(y), y) \in V \times \boldsymbol{V}^{*} ; s \in \mathbb{R}, \operatorname{det}(y) \neq 0\right\}, \\
= & \text { Zariski closure of } \\
& \left\{\left(s \cdot y^{-1}, y\right) \in \boldsymbol{V} \times \boldsymbol{V}^{*} ; s \in R, \operatorname{det}(y) \neq 0\right\} .
\end{aligned}
$$

Hence from (2.44), we have

$$
\sigma=\frac{\langle x, y\rangle}{n}=\frac{s\left\langle y^{-1}, y\right\rangle}{n}=\frac{s \operatorname{tr}\left(y^{-1} y\right)}{n}=s .
$$

We have

$$
P_{A_{n}}=\left.\frac{P \circ \pi}{\sigma^{n}}\right|_{A_{n}}=\frac{s^{n}\left(\operatorname{det} y^{-1}\right)}{s^{n}}=\operatorname{det}(y)^{-1},
$$

and

$$
\begin{aligned}
\omega_{A_{n}}=\frac{\pi^{*}(d x) \bigwedge d \sigma}{\sigma^{n^{\prime}}} /\left.d \sigma\right|_{A_{n}} & =2^{\frac{n(n-1) v}{2}} \cdot \frac{d\left(s \cdot y^{-1}\right)}{s^{n^{\prime}}} \\
& =2^{\frac{n(n-1) v}{2}} \cdot(\operatorname{det}(y))^{-\frac{2 n^{\prime}}{n}} d y .
\end{aligned}
$$


Thus we have the result.

q.e.d.

Let $c_{i j}^{(n)}(s)$ be the coefficient of $|P|_{i}^{s}(x)$ on $\Lambda_{n}^{0 j}=\{0\} \times \mathbb{V}_{j}^{*(n)}$. We have the following formula of the Fourier transforms by (3.35) and (3.44).

$$
\begin{gathered}
\left.(2 \pi)^{n s+\frac{n^{\prime}}{2} \cdot 2^{\frac{n(n-1) v}{2}}}|P|_{i}^{s}(y)\right|_{V_{\boldsymbol{R}}^{*}-s_{\boldsymbol{R}}^{*}} \\
=\sum_{j=0}^{n} 2^{\frac{n(n-1) v}{4}} c_{i j}^{(n)}(s)|P|_{j}^{-s-\frac{n^{\prime}}{n}}(y) .
\end{gathered}
$$

Thus we have

Theorem 3.8 (Formulas of Fourier transforms). Let $s \notin \operatorname{Crit}\left(P(x)^{s}\right)$. Then we have,

$$
\begin{aligned}
& \left.\int|P|_{i}^{s}(x) \exp (-2 \pi \sqrt{-1}\langle x, y\rangle) d x\right|_{V_{\boldsymbol{R}}^{*}-S_{\boldsymbol{R}}^{*}} \\
& =(2 \pi)^{-n s-\frac{n^{\prime}}{2}} \cdot 2^{-\frac{n(n-1) v}{4}} \sum_{j=0}^{n} c_{i j}^{(n)}(s) \mid P_{j}^{-s-\frac{n^{\prime}}{n}}(y),
\end{aligned}
$$

where $n^{\prime}=\operatorname{dim} \boldsymbol{V}$ and $v= \begin{cases}1 & (\mathbb{V}=\boldsymbol{S y m}(n, \mathbb{R})) \\ 2 & (\mathbb{V}=\mathbb{H e r}(n, \mathbb{C})) . \\ 4 & (\mathbb{V}=\boldsymbol{H e r}(n, \mathbb{H}))\end{cases}$

The calculation of the Fourier transform is reduced to the calculation of the coefficient $c_{i j}(s)$. By the formula (2.49) (Chapter I Proposition 2.13), we have the relations of the coefficients on $\Lambda_{i}$ and those on $\Lambda_{i+1}$. Thus we can compute the relations between the coefficients on $\Lambda_{n}$ and those on $\Lambda_{0}$ inductively.

Now, we shall calculate $c_{i j}^{(n)}(s)$. Let $t$ be a variable, and we set $c_{\Lambda_{0}^{i 0}}=t^{i}$. Then we have

$$
c_{\boldsymbol{A}_{n}^{0 j}}=\sum_{i=1}^{n} c_{i j}^{(n)}(s) \cdot c_{\boldsymbol{A}_{0}^{i 0}}=\sum_{i=1}^{n} c_{i j}^{(n)}(s) \cdot t^{i} .
$$

Conversely, from the relation $c_{\Lambda_{n}^{0 j}}=\sum_{i=1}^{n} c_{i j}^{(n)}(s) t^{i}$, we have $c_{\Lambda_{n}^{0 j}}=\sum_{i=1}^{n} c_{i j}^{(n)}(s) c_{\Lambda_{0}^{i 0}}$ for arbitrary coefficients. On the other hand, when we give the associated numbers $c_{\boldsymbol{A}_{0}^{i 0}}=t^{i}$ we have $c_{A_{i}^{j k}}: c_{\boldsymbol{A}_{i}^{j-1 k}}=l: 1$. This is proved by induction on $i$ using the relation matrix (2.49), which does not depend on $j$. Therefore we have from (2.49).

$$
\begin{aligned}
& c_{A_{i+1}^{j-1 k+1}}=F_{i k}^{+}(s) c_{A_{i}^{j-1 k}} \\
& c_{A_{i+1}^{j-1 k}}=F_{i k}^{-}(s) c_{A_{i}^{j-1 k}}
\end{aligned}
$$


with

$$
\begin{aligned}
F_{i k}^{+}(s)=\frac{\Gamma\left(s-s_{i+1}\right)}{\sqrt{2 \pi}}( & \exp \left(-\frac{\pi}{2} \sqrt{-1}\left(s-s_{i+1}\right)\right) \exp \left(-\frac{\pi}{4} \sqrt{-1}(2 k-i) v\right) t \\
& \left.+\exp \left(\frac{\pi}{2} \sqrt{-1}\left(s-s_{i+1}\right)\right) \exp \left(-\frac{\pi}{4} \sqrt{-1}(i-2 k) v\right)\right), \\
F_{i k}^{-}(s)=\frac{\Gamma\left(s-s_{i+1}\right)}{\sqrt{2 \pi}}( & \exp \left(\frac{\pi}{2} \sqrt{-1}\left(s-s_{i+1}\right)\right) \exp \left(-\frac{\pi}{4} \sqrt{-1}(2 k-i) v\right) t \\
& \left.+\exp \left(-\frac{\pi}{2} \sqrt{-1}\left(s-s_{i+1}\right)\right) \exp \left(-\frac{\pi}{4} \sqrt{-1}(i-2 k) v\right)\right) .
\end{aligned}
$$

Then we have

$$
\begin{aligned}
c_{\Lambda_{n}^{0 j}}= & F_{n-1 j-1}^{+}(s) F_{n-2 j-2}^{+}(s) \ldots F_{n-j 0}^{+}(s) \\
& \times F_{n-j-10}^{-}(s) F_{n-j-20}^{-}(s) \ldots F_{00}^{-}(s) c_{\Lambda_{0}^{00}} .
\end{aligned}
$$

$$
\begin{aligned}
& F_{n-1 j-1}^{+}(s) F_{n-2 j-2}^{+}(s) \ldots F_{n-j 0}^{+}(s) \\
& \times F_{n-j-10}^{-}(s) F_{n-j-20}^{-}(s) \ldots F_{00}^{-}(s)
\end{aligned}
$$

is equal to;

$$
\begin{aligned}
& (\sqrt{2 \pi})^{-n} \Gamma(s+1) \Gamma\left(s+\frac{3}{2}\right) \ldots \Gamma\left(s+\frac{n+1}{2}\right) \\
& \times \prod_{p=1}^{j} \exp \left(-\frac{\pi}{2} \sqrt{-1} p\right)\left(t \cdot \exp \left(-\frac{\pi}{2} \sqrt{-1} s\right)\right. \\
& \left.\quad+(-1)^{p} \exp \left(\frac{\pi}{2} \sqrt{-1} s\right)\right) \\
& \times \prod_{q=1}^{n-j} \exp \left(\frac{\pi}{2} \sqrt{-1} q\right)\left(t \cdot \exp \left(\frac{\pi}{2} \sqrt{-1} s\right)+(-1)^{q} \exp \left(-\frac{\pi}{2} \sqrt{-1} s\right)\right)
\end{aligned}
$$

when $\boldsymbol{V}=\boldsymbol{S y} \boldsymbol{m}(n, \boldsymbol{R})$;

$$
\begin{aligned}
& (\sqrt{2 \pi})^{-n} \Gamma(s+1) \Gamma(s+2) \ldots \Gamma(s+n)(\sqrt{-1})^{-n}(-1)^{\frac{n(n+1)}{2}-n j} \\
& \quad \times\left(t \cdot \exp \left(-\frac{\pi}{2} \sqrt{-1} s\right)-\exp \left(\frac{\pi}{2} \sqrt{-1} s\right)\right)^{j} \\
& \quad \times\left(t \cdot \exp \left(\frac{\pi}{2} \sqrt{-1} s\right)-\exp \left(-\frac{\pi}{2} \sqrt{-1} s\right)\right)^{n-j}
\end{aligned}
$$

when $\boldsymbol{V}=\boldsymbol{H e r}(n, \boldsymbol{C})$;

$$
\begin{aligned}
& (\sqrt{2 \pi})^{-n} \Gamma(s+1) \Gamma(s+3) \ldots \Gamma(s+2 n-1)(\sqrt{-1})^{n} \\
& \times\left(t \cdot \exp \left(-\frac{\pi}{2} \sqrt{-1} s\right)-\exp \left(\frac{\pi}{2} \sqrt{-1} s\right)\right)^{j}
\end{aligned}
$$




$$
\times\left(t \cdot \exp \left(\frac{\pi}{2} \sqrt{-1} s\right)-\exp \left(-\frac{\pi}{2} \sqrt{-1} s\right)\right)^{n-j}
$$

when $\mathbb{V}=\operatorname{Her}(n, \boldsymbol{H})$.

Theorem 3.9 (Explicit computations of $c_{i j}(s)$ ).

1. In the case of $V=\mathbb{S y m}(n, \mathbb{R})$.

Putting

$$
\text { (3.55) } \begin{aligned}
c_{i j}^{(n)}(s)= & (2 \pi)^{-\frac{n}{2}} \prod_{p=1}^{n} \Gamma\left(s+\frac{p+1}{2}\right) \exp \left(\frac{\pi}{2} \sqrt{-1}\left(\frac{n}{2}-i\right)(n+1)\right) \\
& \times \exp \left(\frac{\pi}{2} \sqrt{-1}(2 j+2 i-n) s\right) a_{i j}^{(n)}(s)
\end{aligned}
$$

we have

(3.56) If $n \equiv 1$ (mod.2), then:

1) $\quad a_{i j}^{(n)}(s)=(-1)^{(n+j-2) / 2} \sum_{l=\max (0,(j+i-n-1) / 2)}^{\min ((j-1) / 2,(i-1) / 2)}$

$$
\times\left(\begin{array}{c}
\frac{i-1}{2} \\
l
\end{array}\right)\left(\begin{array}{l}
\frac{n-i}{2} \\
\frac{j-1}{2}-l
\end{array}\right) \exp (-4 \pi \sqrt{-1} l s) \exp (-2 \pi \sqrt{-1} s)
$$

if $i \equiv 1$ and $j \equiv 1(\bmod .2)$.

2) $a_{i j}^{(n)}(s)=(-1)^{(n+j+1) / 2} \sum_{l=m a x(0,(j+i-n) / 2)}^{\min (j / 2,(i-1) / 2)}$

$$
\times\left(\begin{array}{c}
\frac{i-1}{2} \\
l
\end{array}\right)\left(\begin{array}{c}
\frac{n-i}{2} \\
\frac{j}{2}-l
\end{array}\right) \exp (-4 \pi \sqrt{-1} l s)
$$

if $i \equiv 1$ and $j \equiv 0(\bmod .2)$ 。

3) $\quad a_{i j}^{(n)}(s)=(-1)^{(n+j-2) / 2} \sum_{l=\max (0,(j+i-n) / 2)}^{\min ((j-1) / 2, i / 2)}$

$$
\times\left(\begin{array}{l}
\frac{i}{2} \\
l
\end{array}\right)\left(\begin{array}{l}
\frac{n-i-1}{2} \\
\frac{j-1}{2}-l
\end{array}\right) \exp (-4 \pi \sqrt{-1} l s)
$$

if $i \equiv 0$ and $j \equiv 1(\bmod .2)$.

4) $\quad a_{i j}^{(n)}(s)=(-1)^{(n+j+1) / 2} \sum_{l=\max (0,(j+i-n-1) / 2)}^{\min (j / 2)}$ 


$$
\times\left(\begin{array}{l}
\frac{i}{2} \\
l
\end{array}\right)\left(\begin{array}{c}
\frac{n-i-1}{2} \\
\frac{j}{2}-l
\end{array}\right) \exp (-4 \pi \sqrt{-1} l s)
$$

if $i \equiv 0$ and $j \equiv 0(\bmod .2)$.

(3.57) If $n \equiv 0$ (mod.2), then:

1) $a_{i j}^{(n)}(s)=(-1)^{(n+j) / 2} \sum_{l=\max (0,(j-n+i) / 2)}^{\min (j / 2, i / 2)}$

$$
\times\left(\begin{array}{c}
\frac{i}{2} \\
l
\end{array}\right)\left(\begin{array}{c}
\frac{n-i}{2} \\
\frac{j}{2}-l
\end{array}\right) \exp (-4 \pi \sqrt{-1} l s)
$$

if $i \equiv 0$ and $j \equiv 0$ (mod. 2)

2) $a_{i j}^{(n)}(s)=0$

if $i \equiv 0$ and $j \equiv 1$ (mod. 2)

3) $a_{i j}^{(n)}(s)=(-1)^{(n+j) / 2} \sum_{l=\max (0,(j+i-3) / 2)}^{\min (j / 2,(i-1) / 2)}$

$$
\begin{aligned}
& \times\left(\begin{array}{c}
\frac{i-1}{2} \\
l
\end{array}\right)\left(\left(\begin{array}{c}
\frac{n-i-1}{2} \\
\frac{j}{2}-l-1
\end{array}\right) \exp (-2 \pi \sqrt{-1} s)-\left(\begin{array}{c}
\frac{n-i-1}{2} \\
\frac{j}{2}-l
\end{array}\right)\right) \\
& \times \exp (-4 \pi \sqrt{-1} l s)
\end{aligned}
$$

if $i \equiv 1$ and $j \equiv 0$ (mod. 2)

4) $a_{i j}^{(n)}(s)=(-1)^{(n+j-1) / 2} \sum_{l=\max (0,(j-n+i) / 2)}^{\min ((j-1) / 2,(i-1) / 2)}$

$$
\begin{aligned}
& \times\left(\begin{array}{c}
\frac{i-1}{2} \\
l
\end{array}\right)\left(\begin{array}{c}
\frac{n-i-1}{2} \\
\frac{j-1}{2}-l
\end{array}\right)(\exp (\pi \sqrt{-1} s) \\
& +\exp (-\pi \sqrt{-1} \mathrm{~s})) \exp (-4 \pi \sqrt{-1} l s)
\end{aligned}
$$

if $i \equiv 1$ and $j \equiv 1(\bmod .2)$

2. In the case of $\boldsymbol{V}=\boldsymbol{H e r}(n, \boldsymbol{C})$.

(3.58) $\quad c_{i j}^{(n)}(s)=(2 \pi)^{-n / 2} \prod_{p=1}^{n} \Gamma(s+p) \exp \left(\frac{\pi}{2} \sqrt{-1}\left(-n^{2}+2 n j\right)\right)$ 


$$
\begin{aligned}
& \times \exp \left(\frac{\pi}{2} \sqrt{-1}(2 j+2 i-n) s\right) \sum_{l=\max (0, j+i-n)}^{\min (j, i)} \\
& \times(-1)^{j}\left(\begin{array}{l}
j \\
l
\end{array}\right)\left(\begin{array}{l}
n-j \\
i-l
\end{array}\right) \exp (-2 \pi l \sqrt{-1} s)
\end{aligned}
$$

3. In the case of $\mathbb{V}=\operatorname{Her}(n, \mathbb{H})$.

$$
\begin{aligned}
c_{i j}^{(n)}(s) & =(2 \pi)^{-n / 2} \prod_{p=1}^{n} \Gamma(s+(2 p-1)) \exp \left(\frac{\pi}{2} \sqrt{-1}(n-2 j)\right) \\
& \times \exp \left(\frac{\pi}{2} \sqrt{-1}(2 j+2 i-n) s\right) \sum_{l=\max (0, i+j-n)}^{\min (i, j)} \\
& \times(-1)^{n+j}\left(\begin{array}{l}
j \\
l
\end{array}\right)\left(\begin{array}{l}
n-j \\
i-l
\end{array}\right) \exp (-2 \pi \sqrt{-1} l s)
\end{aligned}
$$

Theorem 3. 10. Let $s \notin \operatorname{Crit}\left(P(x)^{s}\right)\left(\operatorname{resp} .-s-\left(n^{\prime} / n\right) \notin \operatorname{Crit}\left(P(x)^{s}\right)\right)$.

1) Let $u(x)$ (resp. $v(y)$ ) be a hyperfunction solution to $\mathfrak{M}_{s}$ on $\mathbb{V}_{\boldsymbol{R}}-\mathbb{S}_{\boldsymbol{R}}$ (resp. to $\mathfrak{M}_{s+\left(n^{\prime} / n\right)}^{*}$ on $\mathbb{V}_{\boldsymbol{R}}^{*}-\mathbb{S}_{\boldsymbol{R}}^{*}$ ). Then, $u(x)($ resp. $v(y)$ ) is uniquely extended to $\boldsymbol{V}_{\boldsymbol{R}}\left(\operatorname{resp} . \boldsymbol{V}_{\boldsymbol{R}}^{*}\right)$ as a hyperfunction solution.

2) The hyperfunction $|P|_{i}^{s}(x)$ (resp. $\left.|P|_{i}^{-s-\left(n^{\prime} / n\right)}(y)\right) \quad(i=1, \ldots, n)$ forms a basis of the hyperfunction solution to $\mathfrak{M}_{s}$ on $\mathbb{V}_{\boldsymbol{n}}$ (resp. to $\mathfrak{M}_{s+\left(n^{\prime} / n\right)}^{*}$ on $\mathbb{V}_{R}^{*}$.

Proof. 1) Let $u(x)$ be a hyperfunction solution on $\mathbb{V}_{R}-\mathbb{S}_{R}$ to $\mathfrak{M}_{s}$ with $s \notin \operatorname{Crit}\left(P(x)^{s}\right)$. From Proposition 3.5,3), we have

$$
\left.u(x)=\sum_{i=1}^{n} a_{i} \cdot|P| i \mid s\right)\left.\right|_{V_{R}}-s_{R^{*}}
$$

Since $s \notin \operatorname{Crit}\left(P(x)^{s}\right), \sum_{i} a_{i}|P|_{i}^{s}(x)$ is well defined as a tempered distribution on $\mathbb{V}_{R}$ by Proposition 3.4,1), and hence $u(x)$ is extendable to $\mathbb{V}_{\boldsymbol{R}}$ as a solution of $\mathfrak{M}_{s}$.

Next, we shall show the uniqueness of the extension. Since $u(x)$ is a solution of the linear differential equation $\mathfrak{M}_{s}$, we have to show that if $\left.u(x)\right|_{V_{R}-s_{R}}=0$, then $u(x)=0$ on $\mathbb{V}_{\boldsymbol{R}}$.

If $\left.u(x)\right|_{V_{R}-S_{R}}=0$, then the coefficients on $\mathbb{V}_{j}^{(n)} \times\{0\}, c_{V_{j}^{(n)} \times\{0\}}(u(x))$ are zero for any $j$. Note that the matrices in (2.49) is well defined for any $s \notin \operatorname{Crit}\left(P(x)^{s}\right)$, and for any $0 \leqq i \leqq n-1$. Then we have

$$
c_{\Lambda_{1}^{j k}}(u(x))=0
$$

for any $0 \leqq j \leqq n-1$ and $0 \leqq k \leqq 1$ by the matrix (2.49) with $i=0$. 
Moreover, since the coefficients of $u(x)$ on $\Lambda_{l}^{0}$ are determined by those on $\Lambda_{l-1}^{\circ}$ by the matrices (4.29) with $i=l$, we have that the coefficients of $u(x)$ on $\Lambda_{l}^{\circ}$ are all zero if the coefficients of $u(x)$ on $\Lambda_{l-1}^{\circ}$ are all zero. Thus, by induction on $l$, we have that all the coefficients $c_{A_{i}^{j k}}(u(x))$ are zero. Therefore, by Theorem 2.4, we have $u(x)=0$.

By Proposition $3.5,1)$ this theorem is true for the solution $v(y)$ to $\mathfrak{M}_{s+\left(n^{\prime} / n\right)}^{*}$ on $\mathbb{V}_{\boldsymbol{R}}^{*}$.

2) Any solution $u(x)$ to $\mathfrak{M}_{s}$ on $V_{R}$ is written as

$$
\left.u(x)\right|_{V_{R}-S_{R}}=\left.\sum_{i} a_{i}|P|_{i}^{s}(x)\right|_{V_{R}}-s_{R},
$$

by Proposition $3.5,3$ ) and hence, by 1 ), we have

$$
u(x)=\sum_{i} a_{i}|P|_{i}^{s}(x),
$$

on $\mathbb{V}_{\boldsymbol{R}}$. Thus we have the result. Similarly, we can prove the theorem for a solution $v(y)$ to $\mathfrak{M}_{s+\left(n^{\prime} / n\right)}^{*}$.

$$
\text { q. e. d. }
$$

Theorem 3.11. The formulas of Fourier transforms in Theorem 3.8 are valid not only on $\mathbb{V}_{\boldsymbol{R}}^{*}-\mathbb{S}_{\boldsymbol{R}}^{*}$ but also on $\mathbb{V}_{\boldsymbol{R}}^{*}$ and not only for $s \notin \operatorname{Crit}\left(P(x)^{s}\right)$ but also for all $s \in \mathbb{C}$. That is to say, we have

$$
\begin{aligned}
& \int|P|_{i}^{s}(x) \exp (-2 \pi \sqrt{-1}<x, y>) d x \\
& =(2 \pi)^{-n s-\left(n^{\prime} / 2\right)} 2^{-n(n-1) v / 4} \sum_{j=0}^{n} c_{i j}^{(n)}(s)|P|_{j}^{-s-\left(n^{\prime} / n\right)}(y),
\end{aligned}
$$

for any $s \in C$ by considering $|P|_{i}^{s}(x)$ to be a tempered distribution with a meromorphic parameter $s \in \mathbb{C}$. Here, we use the same notations as in Theorem 3.8.

Proof. We suppose that $s \notin \operatorname{Crit}\left(P(x)^{s}\right)$ and $-s-\left(n^{\prime} / n\right) \notin$ Grit $\left(P(x)^{s}\right)$. Then both $|P|_{i}^{s}(x)$ and $|P|_{j}^{-s-\left(n^{\prime} / n\right)}(y)$ are well defined, and the formula (3.60) is valid on $\mathbb{V}_{\boldsymbol{R}}^{*}-\mathfrak{S}_{\boldsymbol{R}}^{*}$. However, the left hand side of (3.60) is a solution to the holonomic system $\mathfrak{M}_{s+\left(n^{\prime} / n\right)}^{*}$ on $\mathbb{V}_{R}^{*}$ by Proposition 3.5,2), and the right hand side of $(3.60)$ is a solution to $\mathfrak{M}_{s+\left(n^{\prime} / n\right)}^{*}$ on $\mathbb{V}_{\boldsymbol{R}}^{*}-\boldsymbol{S}_{\boldsymbol{R}}^{*}$. Therefore, since $-s-\left(n^{\prime} / n\right) \notin \operatorname{Crit}\left(\mathrm{P}(x)^{s}\right)$, the right hand side is extended uniquely as a solution to $\mathfrak{M}_{s+\left(n^{\prime} / n\right)}^{*}$ on $\boldsymbol{V}_{\boldsymbol{R}}^{*}$ by Theorem 3.10. Thus the formula (3.60) is valid for any $s$ in

$$
A=\left\{s \in \mathbb{C} ; s \notin \operatorname{Crit}\left(P(x)^{s}\right) \text { and }-s-\left(n^{\prime} / n\right) \notin \operatorname{Crit}\left(P(x)^{s}\right)\right\} \text {. }
$$


Since $\mathbb{A}$ is the complement of a discrete set in $\mathbb{C},(3.60)$ is valid for any $s \in \boldsymbol{C}$ by the analytic continuation of Theorem 3.2,1).

q. e.d.

\section{§4. Invariant Measures on Singular Orbits and Their Fourier Transforms}

In this section, we use the term associated numbers instead of coefficients in order to avoid confusions.

Theorem 4.1. Let $\boldsymbol{S}_{i}^{j}=\{x \in \mathbb{V} ; \operatorname{sign}(x)=(j, n-i-j)\}$. There exist hyperfunctions $T_{i s}^{j}(x)(i=0,1, \ldots, n, j=0,1, \ldots, n-i)$ with meromorphic parameter $s \in \mathbb{C}$ satisfying the following properties.

(4.1) 1) $T_{i s}^{j}(\rho(g) x)=\chi(g)^{s} T_{i s}^{j}(x)$ 。

2) For $i \geqq 1$, the support of $T_{i s_{i}}^{j_{i}}(x)$ is contained in $\bar{S}_{i}^{j}$ and $T_{i s_{i}}^{j}(x)$ $d x$ gives a non-zero measure on $\mathbb{S}_{i \circ}^{j}$

3) The associated number of $T_{i s_{i}}^{j}(x)$ on $\mathbb{\Lambda}_{i}^{j k}$ is 1 .

Proof. We define $T_{i s}^{j}(x)$ by induction on $i$. First, we set

$$
T_{0 s}^{j}(x)=|P|_{j}^{s}(x) \quad(j=0,1, \ldots, n) \text {. }
$$

Then $T_{0 s}^{n-p}(x)$ is a hyperfunction whose support is $\mathbb{V}_{n-p}^{(n)}$. Suppose that we have defined $T_{i s}^{j}(x)$ for $i=0,1, \ldots, q$ and $j=0,1, \ldots, n-i$. We define

$$
\begin{aligned}
T_{q+1 s}^{j}(x)= & a_{q}(s)\left(b_{q}(s) T_{q s}^{j+1}(x)-b_{q}(s)^{3} T_{q s}^{j+2}(x)+\ldots\right. \\
& \left.\ldots+(-1)^{n-q-1-j} b_{q}(s)^{2(n-q-j)-1} T_{q s}^{n-q}(x)\right)
\end{aligned}
$$

for $j=0,1, \ldots, n-q-1$. Here, we set

$$
\begin{aligned}
& a_{q}(s)=\sqrt{2 \pi} \Gamma\left(s-s_{q+1}\right)^{-1}, \\
& b_{q}(s)=\exp \left(\frac{\pi}{2} \sqrt{-1}\left(s-s_{q+1}\right)\right) \exp \left(-\frac{\pi}{4} \sqrt{-1} q \cdot v\right)
\end{aligned}
$$

where

$$
v= \begin{cases}1 & (\mathbb{V}=\operatorname{Sym}(n, \mathbb{R})) \\ 2 & (\mathbb{V}=\mathbb{H e r}(n, \mathbb{C})) \\ 4 & (\mathbb{V}=\mathbb{H e r}(n, \mathbb{H})) .\end{cases}
$$


From the definition, $T_{i s}^{j}(x)$ is a linear combination of $|P|_{k}^{s}(x)$ with entire function coefficients on $s \in \boldsymbol{C}$. Therefore $T_{i s}^{j}(x)$ is a hyperfunction solution of $\mathfrak{M}_{s}$, and hence satisfies the condition 1).

Lemma 4.1.1. Let $c_{\mathcal{A}_{i}^{j k}}\left(T_{p s}^{q}(x)\right)$ be the associated number of $T_{p s}^{q}(x)$ on $\Lambda_{i}^{j k}$. Then, for $p \geqq 1$, we have

$$
\begin{aligned}
& \text { 1) }\left.c_{A_{i}^{j k}}\left(T_{p s}^{q}(x)\right)\right|_{s=s_{p}}=0 \text { if } \quad i<p . \\
& \text { 2) }\left.c_{A_{p}^{j k}}\left(T_{p s}^{q}(x)\right)\right|_{s=s_{p}}= \begin{cases}1, & j=q, \\
0, & j \neq q .\end{cases} \\
& \text { 3) }\left.c_{A_{i}^{j k}}\left(T_{p s}^{q}(x)\right)\right|_{s=s_{p}}=0 \text { if } i>p \text { and } j<q-(i-p) \\
& \text { or if } i>p \text { and } j>q .
\end{aligned}
$$

Proof. First, in order to prove (4.5), we shall show that the associated numbers of $T_{p s}^{q}(x)$ on $\Lambda_{i}^{j k}$ are entire functions in $s$ and that they vanish at $s=s_{p}$ if $i<p$. We shall show these by induction on $p$. Consider the hyperfunction $a_{0}(s)|P|_{q}^{s}(x)(q=0,1, \ldots, n)$. Their associated numbers on $\boldsymbol{\Lambda}_{0}^{j 0}(j=0,1, \ldots, n)$ are

$$
\begin{cases}a_{0}(s) & (q=j), \\ 0 & (q \neq j) .\end{cases}
$$

They all vanish at $s=s_{1}$ because $a_{0}(s)$ is an entire function with the zero of order 1 at $s=s_{1}$. Therefore the associated numbers of $T_{1 s}^{k}(x)$ also vanish on all $\Lambda_{0}^{j 0}$ because $b_{0}(s)$ is an entire function. The associated numbers on $\Lambda_{1}^{j k}(j=0,1, \ldots, n-1, k=0,1)$ are all entire functions on $s \in \mathbb{C}$, since they can be written as $a_{0}(s) . \quad \Gamma\left(s-s_{1}\right) \times$ (an entire function) from the relations of the associated numbers (2.49). Thus we obtain that our assertion is valid for $p=1$.

Suppose that

(4.7) 1) The associated numbers of $T_{p s}^{q}(x)$ on $\Lambda_{i}^{j k}$ are entire function on $s \in \boldsymbol{C}$ when $i \leqq p$,

2) They vanish at $s=s_{p}$ if $i<p$.

Consider the hyperfunction $a_{p}(s) T_{p s}^{q}(x)$. Since $a_{p}(s)$ is an entire function with a zero of order 1 at $s=s_{p+1}$, the associated numbers on all $\boldsymbol{\Lambda}_{i}^{j k}(i \leqq p)$ are entire functions in $s \in \boldsymbol{C}$ and vanish at $s=s_{p+1}$. Moreover, the associated numbers on $\Lambda_{i+1}^{j k}$ are entire functions in $s$ 
because they can be written as $a_{p}(s) \cdot \Gamma\left(s-s_{p+1}\right) \times($ an entire function). Since $T_{p+1 s}^{q}(x)$ are written as a linear combination of $a_{p}(s) T_{p s}^{q}(x)$ $(q=0, \ldots, n-p)$ with coefficients of entire functions, we obtain that

(4.8) 1) The associated numbers of $T_{p+1 s}^{q}(x)$ on $\Lambda_{i}^{j k}(i \leqq p+1)$ are entire functions on $s \in \mathbb{C}$.

2) They vanish at $s=s_{q+1}$ if $i \leqq p$.

Thus the assertion (4.5) 1) has been proved by induction.

Next we shall show that

(4.9) The associated numbers $\left.c_{\Lambda_{p}^{j k}}\left(T_{p s}^{q}(x)\right)\right|_{s=s_{p}}(0 \leqq k \leqq p)$ coincide with one another.

Note that $\Gamma\left(s-s_{p}\right) c_{A_{p-1}^{j+1 k}}\left(T_{p s}^{q}(x)\right)$ and $\Gamma\left(s-s_{p}\right) c_{A_{p-1}^{j k}}\left(T_{p s}^{q}(x)\right)$ are holomorphic at $s=s_{p}$. We denote by $a_{1}$ and $a_{2}$ the values of them at $s=s_{p}$, respectively. From the relation matrix of associated numbers $(2,49)$, we have

$$
\begin{aligned}
& \left.c_{\Lambda_{p}^{j k+1}}\left(T_{p s}^{q}(x)\right)\right|_{s=s p}=\left.c_{\Lambda_{p}^{j k}}\left(T_{p s}^{q}(x)\right)\right|_{s=s p} \\
& =(\sqrt{2 \pi})^{-1}\left(a_{1}+a_{2}\right) .
\end{aligned}
$$

Thus we have that $\left.c_{A_{p}^{j k}}\left(T_{p s}^{q}(x)\right)\right|_{s=s_{p}}(0 \leqq k \leqq p)$ coincide with one another.

We shall show that

$$
c_{\Lambda_{p}^{j p}}\left(T_{p s}^{q}(x)\right)= \begin{cases}1, & j=q, \\ 0, & j \neq q .\end{cases}
$$

by induction on $p$. When $p=0$, it is evident from the definition. We assume that (4.11) is valid for an integer $p$. Then the associatep numbers $c_{\Lambda_{p}^{j p}}\left(T_{p s}^{k}\right)$ are all identically zero if $k \geqq j+1$, and $c_{A_{p}^{j+1 p}}\left(T_{p s}^{k}\right)$ are all identically zero if $k \geqq j+2$. Therefore we have

$$
\begin{aligned}
& c_{A_{p+1}^{j p+1}}\left(T_{p+1 s}^{j}\right) \\
& =\frac{\Gamma\left(s-s_{p+1}\right)}{\sqrt{2 \pi}} \exp \left(-\frac{\pi}{2} \sqrt{-1}\left(s-s_{p+1}\right)\right) \exp \left(\frac{\pi}{4} \sqrt{-1} p v\right) \\
& \times a_{p}(s) b_{p}(s) c_{A_{p}^{j+1 p}}\left(T_{p s}^{j+1}\right) \\
& =c_{A_{p}^{j+1 p}}\left(T_{p s}^{j+1}\right)=1,
\end{aligned}
$$

and

$$
c_{\boldsymbol{A}_{p+1}^{k p+1}}\left(T_{p+1 s}^{j+1}\right)=0 \text { for } k<j
$$


from the induction hypothesis. For $k>j$, the associated number of $T_{p+1 s}^{j}$ on $\boldsymbol{\Lambda}_{p+1}^{k p+1}$ are determined by the associated numbers on $\boldsymbol{\Lambda}_{p}^{k+1 p}$ and $\boldsymbol{\Lambda}_{p}^{k p}$. We have

$$
\begin{aligned}
& C_{\boldsymbol{A}_{p+1}^{k p+1}}^{k+1}\left(T_{p+1 s}^{j}\right) \\
& =\frac{\Gamma\left(s-s_{p+1}\right)}{\sqrt{2 \pi}}\left(\exp \left(-\frac{\pi}{2} \sqrt{-1}\left(s-s_{p+1}\right)\right) \exp \left(\frac{\pi}{4} \sqrt{-1} p v\right)(-1)^{k-j}\right. \\
& \times a_{p}(s) b_{p}(s)^{2 k-2 j+1} c_{\Lambda_{p}^{k+1 p}}\left(T_{p s}^{k+1}\right) \\
& \quad+\exp \left(\frac{\pi}{2} \sqrt{-1}\left(s-s_{p+1}\right)\right) \exp \left(-\frac{\pi}{4} \sqrt{-1} p v\right)(-1)^{k-j-1} \\
& \left.\quad \times a_{p}(s) b_{p}(s)^{2 k-2 j-1} c_{\mathcal{A}_{p}^{k p}}\left(T_{p s}^{k}\right)\right) \\
& =(-1)^{k-j} b_{p}(s)^{2 k-2 j}\left(c_{A_{p}^{k+1 p}}\left(T_{p s}^{k+1}\right)-c_{\boldsymbol{A}_{p}^{k p}}\left(T_{p s}^{k}\right)\right) \\
& =0
\end{aligned}
$$

for $k>j$. Thus we have

$$
c_{A_{p+1}^{k p+1}}\left(T_{p+1 s}^{j}\right)= \begin{cases}1 & k=j, \\ 0 & k \neq j .\end{cases}
$$

Thus we complete the proof by induction. By (4.9) and (4.11), we have the assertion (4.5) 2).

Now we shall show (4.5) 3) by induction on $i$. From (4.5) 2 ) and the relation matrix of associated numbers, we have

$$
c_{A_{p+1}^{j k}}\left(T_{p s}^{q}\right)_{s=s_{p}}=0
$$

if $j<q-1$ or $j>q$. Suppose that for a fixed $i,\left.c_{A_{i}^{j k}}\left(T_{p s}^{q}(x)\right)\right|_{s=s_{p}}=0$ if $j<q-i+p$ or $j>q$. Then, the associated number $\left.c_{A_{i+1}^{j k}}\left(T_{p s}^{q}(x)\right)\right|_{s=s}$ $(j<q-(i+1-p)$ or $j>q)$ are written as linear combinations of the associated numbers on $\boldsymbol{\Lambda}_{i}^{j k}(j<q-(i-p)$ or $j>q)$. Then we have

$$
\left.c_{A_{i+1}^{j k}}\left(T_{p s}^{q}(x)\right)\right|_{s=s_{p}}=0
$$

if $j<q-(i+1-p)$ or $j>q$. Thus by induction we have (4.5) 3).

Lemma 4.1 .1 q.e.d.

From Lemma 4.1.1, the singular spectrum of $T_{p s_{i}}^{q}(x)$ is contained in

$$
\bigcup_{i=0}^{\overline{n-p}}\left(\bigcup_{i \geq j \geqq 0}\left(\bigcup_{k=0}^{p+i} \Lambda_{p+i}^{q-j k}\right)\right) .
$$


Therefore the support of $T_{p s_{i}}^{q}(x)$ is contained in

$$
\begin{aligned}
\left.\pi \overline{\left(\bigcup_{i=0}^{n-p}\left(\bigcup_{i \geqq j \geqq 0}\left(\bigcup_{k=0}^{p+i} \Lambda_{p+i}^{q-j, k}\right)\right)\right.}\right) & =\boldsymbol{S}_{p}^{q} \cup\left(\boldsymbol{S}_{p+1}^{q} \cup \mathbb{S}_{p+1}^{q-1}\right) \cup \ldots \cup\left(\boldsymbol{S}_{n}^{0}\right) \\
& =\overline{\boldsymbol{S}}_{p}^{q}
\end{aligned}
$$

by Proposition 2.3. Thus we have $\operatorname{Supp}\left(T_{p s_{p}}^{q}(x)\right) \subset \bar{S}_{p}^{q}$ by $(2.42)$.

Lemma 4.1.2. Let $p$ be a point in $\mathbb{V}$ and let $\mathbb{K}$ be a non-singular variety in $\mathbb{V}$ of codimension $m$ defined in a neighborhood of $p$. Suppose that a hyperfunction $u(x)$ defined in a neighborhood of $p$ satisfies $\operatorname{Supp}(u(x))=\mathbb{K}$.

Let $\boldsymbol{\Lambda}$ be a Lagrangian variety $\mathbb{T}_{\boldsymbol{K}}^{*} \boldsymbol{V}$, and we denote by $\mathbb{A}_{C}$ its complexification. We suppose that the hyperfunction $u(x)$ satisfies a holonomic system whose characteristic variety contains $\mathbb{A}_{C}$, and the holonomic system is simple on $\mathbb{A}_{c}$ and the order of $u(x)$ is $m / 2$. Then there exists a local coordinate system $\left(x_{1}, \ldots, x_{n^{\prime}}\right)$ near $p$ satisfying

i) $\mathbb{K}=\left\{x_{1}=\ldots=x_{m}=0\right\}$.

ii) $u(x)=P\left(x_{m+1}, \ldots, x_{n^{\prime}}\right) \delta\left(x_{1}\right) \ldots \delta\left(x_{m}\right)$, where $P\left(x_{m+1}, \ldots, x_{n^{\prime}}\right)$ is a non zero real analytic function.

Proof. Since the hyperfunction $u(x)$ satisfies simple holonomic system on $\Lambda_{c}$, we can write as a microfunction $u(x)=P\left(x, D_{x}\right) \delta\left(x_{1}\right) \ldots$ $\delta\left(x_{m}\right)$ on $\Lambda$ by using a local coordinate system $\left(x_{1}, \ldots, x_{n^{\prime}}\right)$ satisfying $K=\left\{x_{1}=\ldots=x_{m}=0\right\}$, and a microdifferential operator of fractional order $P\left(x, D_{x}\right)$. We may assume that $P\left(x, D_{x}\right)$ is written as $P\left(x_{m+1}, \ldots\right.$, $\left.x_{n^{\prime}}, D_{x_{1}}, \ldots, D_{x_{m}}\right)$ and it is uniquely determined by this expression (see the definition of real principal symbol (2.43)). Since the support of $u(x)$ is contained in $\boldsymbol{K}, P\left(x, D_{x}\right)$ is a proper differential operator, i. e., without any terms of order less than -1 and with terms of integer order. The order of $u(y)$ on $\Lambda$ is $\operatorname{ord}(\sigma(P))+\frac{m}{2}$, and hence $P$ is of order 0 , i. e., an analytic function. Therefore we obtain the expression

$$
u(x)=P\left(x_{m+1}, \ldots, x_{n}\right) \delta\left(x_{1}\right) \ldots \delta\left(x_{m}\right),
$$

with some analytic function in a neighborhood of $p$.

Lemma 4.1.2 q.e.d. 
We apply this Lemma to $T_{p s_{p}}^{q}(x)$. Let $z \in \boldsymbol{S}_{p}^{q}$. Then $\boldsymbol{S}_{p}^{q}$ is a nonsingular subvariety in a neighborhood of $z$, and the codimension is $p(p+1) / 2 \quad\left(\right.$ resp. $\left.p^{2}, p(2 p-1)\right)$ when $\boldsymbol{V}=\boldsymbol{S y m}(n, \boldsymbol{R}) \quad($ resp. $\mathbb{V}=$ $\operatorname{HHer}(n, \boldsymbol{C}), \boldsymbol{V}=\operatorname{Her}(n, \mathbb{H}))$. The order of $T_{p s_{p}}^{q}(x)$ on $T_{s_{p}^{q}}^{*} \boldsymbol{V}$ is $-p s_{p}-$ $\frac{p(p+1)}{4}-\left(\right.$ resp. $\left.-p s_{p}-\frac{p^{2}}{2},-p s_{p}-p(2 p-1)\right)$ and hence coincides with a half of the codimension of $S_{p}^{q}$. Therefore Lemma 4.1 .2 can be applied, and hence we have the result (4.1) 2).

q. e. d.

We define the hyperfunction

$$
T_{i}^{j}(x):=T_{i s_{i}}^{j}(x) .
$$

This hyperfunction is relatively invariant and its support coincides with $\bar{S}_{i}^{j}$ and it defines a $\boldsymbol{G}^{1}$-invariant measure on $\boldsymbol{S}_{i \text { 。 }}^{j}$ That is to say, we have the following theorem.

Theorem 4.2. There exists a $\boldsymbol{G}^{1}$-invariant measure $d \nu_{i}^{j}$ on $\boldsymbol{S}_{i}^{j}$ and satisfies

$$
\int_{V} f(x) T_{i}^{j}(x) d x=\left.\int_{\boldsymbol{s}_{i}^{j}} f\right|_{\boldsymbol{S}_{i}^{j}}(x) d \nu_{i}^{j}(x),
$$

for any $f(x) \in C_{0}^{\infty}(V)$ such that $\left.f\right|_{\boldsymbol{S}_{i}^{j}} \in C_{0}^{\infty}\left(\boldsymbol{S}_{i}^{j}\right)$.

Conversely, for any $G^{1}$-invariant measure $d \nu_{i}^{j}$ on $\mathbb{S}_{i}^{j}$,

$$
\left.\int_{\boldsymbol{s}_{i}^{j}} f\right|_{\boldsymbol{S}_{i}^{j}}(x) d \nu_{i}^{j}(x)
$$

is absolutely convergent and coincides with

$$
\text { (const.) } \int_{V} f(x) T_{i}^{j}(x) d x,
$$

for any $f(x) \in \mathscr{S}(\mathbb{V})$.

Proof. In this proof, we denote $G^{(n) 1}$ and $\mathbb{V}^{(n)}$ instead of $G^{1}$ and $\mathbb{V}$, respectively for the later convenience.

The formula (4.21) is the direct consequence of Theorem 4.1. Namely, $T_{i}^{j}(x)$ is a hyperfunction on $\mathbb{V}$, whose support is contained in $\bar{S}_{i}^{j}$. Therefore,

$$
f \longmapsto \int f(x) T_{i}^{j}(x) d x,\left(f \in C_{0}^{\infty}(\mathbb{V}),\left.f\right|_{\boldsymbol{s}_{i}^{j}} \in C_{0}^{\infty}\left(\boldsymbol{S}_{i}^{j}\right)\right),
$$


gives a measure on $S_{i}^{j}$. It follows from that $T_{i}^{j}(\rho(g) x) d(\rho(g) x)=$ $T_{i}^{j}(x) d x\left(g \in G^{(n) 1}\right)$ that this measure is a $G^{(n) 1}$-invariant measure.

Next we shall show the converse.

We consider the relatively invariant measure $d x^{(n)}$ on $\mathbb{V}_{i}^{(n)}$. By Sato-Shintani [5] p. 138, there exists a $G^{(n) 1}$-invariant $n^{\prime}-1$ form $\omega_{n}(x)$ on $V_{i}^{(n)}$ such that

$$
\left|d(P(x)) \wedge \omega_{n}(x)\right|=d x^{(n)} .
$$

Here, $n^{\prime}$ is the dimension of $\mathbb{V}^{(n)}$ and $P(x)$ is the restriction of the relative invariant defined in (1.2). Then, for any $f(x) \in \mathscr{S}\left(\mathbb{V}^{(n)}\right)$, we have

$$
\begin{aligned}
& \int_{V_{i}^{(n)}} f(x)|P(x)|^{s} d x^{(n)} \\
& =\int_{0}^{\infty} t^{s} d t \int_{\left\{x \in V_{i}^{(n)} ;|\operatorname{det}(x)|=t\right\}} f(x) \omega_{n}(x),
\end{aligned}
$$

and they are absolutely convergent when $\operatorname{Re}(s)>-1$.

Let $f(x) \in \mathscr{S}\left(\mathbb{V}^{(n)}\right)$. We put

$$
f^{c o}(x)=\int_{U^{(n)} \ni g} f(\rho(g) x) d g \times \frac{1}{\operatorname{Volume}\left(\mathbb{U}^{(n)}\right)},
$$

where $\mathbb{U}^{(n)}$ is the compact group $\mathbb{O}(n, \mathbb{R}) \cap \mathbb{G}^{(n) 1}(\operatorname{resp} . \mathbb{U}(n, \mathbb{C}), \mathbb{U}(n, \mathbb{H}))$ in the case of $\mathbb{V}^{(n)}=\operatorname{Sym}(n, \mathbb{R}) \quad(\operatorname{resp} \operatorname{Her}(n, \mathbb{C})$, $\mathbb{H e r}(n, \mathbb{H}))$, and $d g$ stands for a $\mathbb{U}^{(n)}$-invariant measure. Then $f^{c o}(x)$ also belongs to $\mathscr{S}\left(\mathbb{V}^{(n)}\right)$, and $\int f^{c o}(x) d x^{(n)}=\int f(x) d x^{(n)}$.

We put

$$
\left.M_{t}^{(i, j)}=\left\{\begin{array}{ll}
\mathbb{L} & 0 \\
0 & 0
\end{array}\right) ; \begin{array}{l}
\mathbb{L} \in \mathbb{S} \boldsymbol{H} \boldsymbol{H}(n, \mathbb{H})),|p(\mathbb{L})|=t, \operatorname{sign}(\mathbb{L}) \\
=(j, n-i-j)
\end{array}\right\}
$$

Then there exists a $G^{(n) 1}$-invariant measure $\omega_{n-i}$ on $M_{t}^{(i, j)}$ given by (4.21). We put

$$
\tilde{M}_{t}^{(i, j)}=\left\{g \cdot M_{t}^{(i, j)} \cdot{ }^{t} \bar{g} ; g \in \mathbb{U}^{(n)}\right\} 。
$$

We can regard $\tilde{M}_{t}^{(i, j)}$ as

$$
\mathbb{U}^{(n)} /\left(\mathbb{U}^{(n-i)} \times \mathbb{U}^{(i)}\right) \cap \mathbb{U}^{(n)} \times M_{t}^{(i, j)} .
$$

Here $\mathbb{U}^{(n-i)} \times \mathbb{U}^{(i)}$ is a subgroup of $\mathbb{U}^{(n)}$ by

$$
\left\{\left(\begin{array}{cc}
A & 0 \\
0 & B
\end{array}\right) ; A \in \mathbb{U}^{(n-i)}, B \in \mathbb{U}^{(i)}\right\} .
$$


There exists a $\boldsymbol{U}^{(n)}$-invariant measure $\omega$ on $\boldsymbol{U}^{(n)} /\left(\boldsymbol{U}^{(n-i)} \times \boldsymbol{U}^{(i)}\right) \cap \boldsymbol{U}^{(n)}$ and we introduce a measure $\tilde{\omega}_{n-i}$ on $\tilde{M}_{t}^{(i, j)}$ by

$$
\tilde{\omega}_{n-i}=\left|\omega \wedge \omega_{n-i}\right| \text {. }
$$

Note that

$$
\boldsymbol{S}_{i}^{j}=\bigcup_{t>0} \tilde{M}_{t}^{(i, j)}
$$

Lemma 4.2.1. The measure

$$
\left|\tilde{\omega}_{n-i} \wedge t^{-1 / 2} d t\right| \quad\left(\operatorname{resp} .\left|\tilde{\omega}_{n-i} \wedge d t\right|,\left|\tilde{\omega}_{n-i} \wedge t d t\right|\right)
$$

is a $\boldsymbol{G}^{(n) 1}$-invariant measure on $\boldsymbol{S}_{i}^{j}$.

Proof. We put $(A, B)=\left(\begin{array}{cc}A & 0 \\ 0 & B\end{array}\right) \in G^{(n) 1}$. Then, by the action of $(A, B)$, the volume form $\left|\tilde{\omega}_{n-i} \wedge d t\right|$ is multiplied by the factor $|\operatorname{det} A|^{-1}$ (resp. 1, $\left.|\operatorname{det} A|^{2}\right)$. Therefore, $\left|\tilde{\omega}_{n-i} \wedge \mathrm{dt}\right|$ is invariant by the action of $(A, B)$. From the definition, it is invariant under the actions of $\boldsymbol{U}^{(n)}$. Since any point in $\boldsymbol{S}_{i}^{j}$ is reduced to the point $\left(\begin{array}{ll}I_{n-i}^{j} & \\ & 0\end{array}\right)$ by the actions of an $(A, B)$ and an element in $\boldsymbol{U}^{(n)}$, and since there exists a $G^{(n) 1}$-invariant measure on $S_{i}^{j}$, the measure $\left|\tilde{\omega}_{n-i} \wedge t^{a} d t\right|$ with $a=-1 / 2(\operatorname{resp} . a=0, a=1)$ is a $G^{(n) 1}$-invariant measure on $\boldsymbol{S}_{i}^{j}$.

$$
\text { q. e. d. of Lemma 4.2.1 }
$$

Now let $f \in \mathscr{S}\left(\mathbb{V}^{(n)}\right)$ and define $f^{c o}(x)$ by (4.26). Then $f^{c o}(x)$ is invariant by the actions of $\boldsymbol{U}^{(n)}$ and

$$
\begin{aligned}
\int_{\tilde{M}_{t}^{(i, j)}} f(x) \tilde{\omega}_{n-i}(x)= & \int_{\tilde{M}_{t}^{(i, j)}} f^{c o}(x) \tilde{\omega}_{n-i}(x) \\
= & \operatorname{Vol}\left(\boldsymbol{U}^{(n)} /\left(\boldsymbol{U}^{(n-i)} \times \boldsymbol{U}^{(i)}\right) \cap \boldsymbol{U}^{(n)}\right) \\
& \times\left.\int_{M_{t}^{(i, j)}} f^{c o}(x)\right|_{\boldsymbol{V}^{(n-i)}} \boldsymbol{\omega}_{n-i}(x) .
\end{aligned}
$$

Here, by the inclusion map

$$
\underset{\prod_{\boldsymbol{V}^{(n-i)}}^{x \longmapsto}}{\stackrel{\prod^{(n)}}{\longrightarrow}\left(\begin{array}{ll}
x & 0 \\
0 & 0
\end{array}\right),}
$$

$\mathbb{V}^{(n-i)}$ can be viewed as a subspace of $\mathbb{V}^{(n)}$. The restriction of $f^{c o}(x)$ on $\mathbb{V}^{(n-i)}$ is an element of $\mathscr{S}\left(\boldsymbol{V}^{(n-i)}\right)$ and it is $\boldsymbol{U}^{(n-i)}$-invariant. 
Lemma 4. 2. 2. Let $f \in \mathscr{S}\left(\mathbb{V}^{(n)}\right)$. Then,

$$
\tilde{f}_{i}(t)=\int_{\left\{x \in V_{i}^{(n)} ;|P(x)|=t\right\}} f(x) \omega_{n}(x)
$$

is a $C^{\infty}$-function on $t>0$ and rapidly decreasing at infinity. Moreover,

$$
\tilde{f}_{i}(0)=\lim _{t \rightarrow 0} \tilde{f}_{i}(t)
$$

exists and is finite.

Proof. In fact, $\tilde{f}_{i}(t)$ is absolutely convergent for $t>0$, and

$$
\frac{\partial}{\partial t} \tilde{f}_{i}(t)=\left(\frac{\widetilde{\partial}}{\partial P(x)} f\right)_{i}(t),
$$

where

$$
\text { (4.39) } \frac{\partial}{\partial P(x)}=|\operatorname{grad} P(x)|^{-2}\left\langle\operatorname{grad} P(x), \frac{\partial}{\partial x}\right\rangle \text {. }
$$

Since $\frac{\partial}{\partial P(x)} f(x)$ is a $C^{\infty}$-function except at the origin and since $\frac{\partial}{\partial P(x)} f(x)$ is rapidly decreasing at infinity, $\left(\frac{\widetilde{\partial}}{\partial P(x)} f\right)_{i}(t)$ is absolutely convergent for $t>0$, and hence it is continuous in $t$ and rapidly decreasing at infinity. In the same way, we can show that $\left(\frac{\partial}{\partial t}\right)^{n} \tilde{f}_{i}(t)$ is continuous on $t>0$ and rapidly decreasing at infinity.

Now we shall show (4.37) by induction on $n$. Suppose that (4.37) is proved for $n \leqq k$. We put $n=k+1$. From the definition, $\lim _{t \rightarrow 0} \tilde{f}_{i}(t)$ coincides with

$$
\int_{\bar{V}_{i}^{(k+1)}-V_{i}^{(k+1)}} f(x) d \nu_{1}(x),
$$

where $d \nu_{1}$ is a $G^{(k+1) 1}$-invariant measure on the orbit of codimension one $S_{1}=\left\{x \in V^{(k+1)} ; P(x)=0, \operatorname{rank}(x)=k\right\}$ which is naturally induced by $\widetilde{\omega}_{k}$, the $(k+1)^{\prime}-1$ form defined by (4.24). From Lemma 4.2.1 and (4.34), the integrability of $\int_{s_{1}^{j}} f(x) d \nu_{1}^{j}$ is reduced to the integrability of

$$
\left.\int_{0}^{\infty} t^{a} d t \int_{M_{t}^{(1, j)}} f^{c o}(x)\right|_{V} ^{(k)} \omega_{k}(x)
$$

with $a=-1 / 2$ (resp. $a=0, a=1$ ). From the induction hypothesis, 


$$
\left.\int_{M_{t}^{(1, j)}} f^{c o}(x)\right|_{V^{(k)}} \omega_{k}(x)=\left(\left.\widetilde{f^{c o}(x)}\right|_{V^{(k)}}\right)_{j}(t)
$$

is a bounded $C^{\infty}$-function which is rapidly decreasing at infinity. Hence (4.40) is integrable.

q. e. d. of Lemma 4.2.2

From Lemma 4.2.1 and (4.34), the integrability of $\int_{s_{i}^{j}} f(x) d \nu_{i}^{j}$ is reduced to the integrability of

$$
\left.\int_{0}^{\infty} t^{a} d t \int_{M_{t}^{(i, j)}} f^{c o}(x)\right|_{V^{(n-i)}} \omega_{n-i}(x)
$$

where $a=-1 / 2$ (resp. $a=0, a=1$ ). From Lemma 4.2.2,

$$
\left(\left.f^{c o}(x)\right|_{V^{(n-i)}}\right)_{j}=\left.\int_{M_{t}^{(i, j)}} f^{c o}(x)\right|_{V^{(n-i)}} \omega_{n-i}(x)
$$

is a bounded $C^{\infty}$-function on $t>0$ and rapidly decreasing at infinity, hence (4.42) is absolutely integrable. Thus,

$$
\left.f \longmapsto \int_{\boldsymbol{S}_{i}^{j}} f(x)\right|_{\boldsymbol{s}_{i}^{j}} d \nu_{i}^{j}(x) \quad\left(f \in \mathscr{S}\left(\boldsymbol{V}^{(n)}\right)\right)
$$

defines a hyperfunction which is also a tempered distribution with support $\overline{\boldsymbol{S}}_{i}^{j}$. We put it $T_{i}^{j^{\prime}}(x)$. From (4.1) 1), 2), the hyperfunction $T_{i}^{j}(x) d x$ defines a $\boldsymbol{G}^{(n) 1}$-invariant measure on $\boldsymbol{S}_{i}^{j}$, and hence

$$
T(x)=T_{i}^{j}(x)-T_{i}^{j^{\prime}}(x)
$$

is a hyperfunction whose support is contained in $\overline{\boldsymbol{S}}_{i}^{j}-\boldsymbol{S}_{i}^{j}$. Moreover $T(x)$ is a solution of $\mathfrak{M}_{s_{i}}$ because we have $T_{i}^{j}(\rho(\mathrm{g}) x)=\chi(g){ }^{s_{i}} T_{i}^{j}(x)$. If $T(x)$ is not zero, the singular spectrum of $T(x)$ is contained in $\cup_{k>i} \overline{T_{S_{k}}^{*} V_{R}}$

Lemma 4. 2.3. Let $u(x)$ be a hyperfunction solution to $\mathfrak{M}_{s_{i}}$. Suppose that $\operatorname{Supp}(u(x)) \subset \bar{S}_{i+1}$. Then $u(x)=0$.

Proof. Since $u(x)$ is a hyperfunction solution to $\mathfrak{M}_{s_{i}}$, it is determined by the associated numbers on $\mathbb{A}_{i}^{j k}$. (Theorem 2.14). From the assumption, the support of $u(x)$ is contained in $\overline{\boldsymbol{S}_{i+1}}$, and hence $u(x)$ is zero on the real Lagrangian subvariety $\boldsymbol{\Lambda}_{j}^{\circ}$ for $0 \leqq j \leqq i$. Therefore, the associated numbers $c_{\boldsymbol{A}_{j}^{k l}}(u(x))=0$ for $0 \leqq j \leqq i$ and for any $k, l$. On 
the other hand, the relation matrix $(2.49)$ is well defined if $s>s_{i+1}$. Since $s_{i}>s_{i+1},(2.49)$ is well defined for $s=s_{i}$, and hence we have

$$
c_{A_{i+1}^{k l}}(u(x))=0 \text {, }
$$

for any $k, l$. Next, consider the matrix (2.49) substituting $i+1$ to $i$. Then, it is well defined for $s=s_{i}$ because $s_{i}>s_{i+2}$. Hence we have $c_{\boldsymbol{A}_{i+2}^{k l}}(u(x))=0$ for any $k, l$ by $(4.44)$. Similarly, by induction, we have $c_{\Lambda_{j}^{k l}}(u(x))=0$ for any $j$ and for any $k, l$. After all, we have $u(x)=0$ by Theorem 2.14.

$$
\text { q.e.d. }
$$

Note that $T(x)$ satisfies the condition of Lemma 4.2.3. In fact,

$$
\overline{\mathfrak{S}}_{i}^{j}-\mathfrak{S}_{i}^{j} \subset{\overline{S_{i+1}}}_{\text {, }}
$$

by Proposition 2.3, and hence the support of $T(x)$ is contained in $\overline{\mathbb{S}}_{i}$. Then, by applying Lemma 4.2.3, we have $T(x)=0$. Therefore we have

$$
T_{i}^{j}(x)=T_{i}^{j^{\prime}}(x)
$$

Thus we have the result.

$$
\text { q. e. d. }
$$

The hyperfunction $T_{i}^{j}(x)$ is a solution of a holonomic system $\mathfrak{M}_{s_{i}}$. When we give associated numbers on $\mathbb{A}_{i}$ of a solution, then we can calculate the associated numbers on $\mathbb{1}_{i+1}$, and inductively we know the associated numbers on $\mathbb{A}_{i+2}, \ldots, \mathbb{A}_{n}$. In order to calculate these associated numbers, we set $c_{i}^{j k}=t^{j}$, where $c_{i}^{j k}$ is the associated number on $\mathbb{\Lambda}_{i}^{j k}$. Then we have

$$
c_{i^{\prime}}^{j k}: c_{i^{\prime}}^{j-1 k}=t: 1 \quad\left(i^{\prime}>i\right),
$$

inductively. We define $F_{i^{\prime} k}^{ \pm}(s)$ as defined in (3.29). We have

$$
\begin{aligned}
& c_{i^{\prime}+1}^{j-1 k+1}=F_{i^{\prime} k}^{+}\left(s_{i}\right) c_{i^{\prime}}^{j-1 k} \quad\left(i^{\prime} \geqq i\right), \\
& c_{i^{\prime}+1}^{j-1 k}=F_{i^{\prime} k}^{-}\left(s_{i}\right) \cdot c_{i^{\prime}}^{j-1 k} \quad\left(i^{\prime} \geqq i\right)
\end{aligned}
$$

from the relation matrix of associated numbers (2. 49).

Therefore we have

$$
\begin{aligned}
c_{n}^{0 k} & =F_{n-1 k-1}^{+}\left(s_{i}\right) \cdots F_{n-k 0}^{+}\left(s_{i}\right) F_{n-k-10}^{-}\left(s_{i}\right) \cdots F_{i 0}^{-}\left(s_{i}\right) \cdot c_{i}^{00} \\
& =\sum_{j=0}^{n-i} a_{i k}^{j} t^{j} c_{i}^{00}=\sum_{j=0}^{n-i} a_{i k}^{j} c_{i}^{j 0}, \quad(k<n-i) .
\end{aligned}
$$




$$
\begin{aligned}
c_{n}^{0 k} & =F_{n-1 k-1}^{+}\left(s_{i}\right) \cdots F_{i k-n+i}^{-}\left(s_{i}\right) c_{i}^{0 k-n+i} \\
& =\sum_{j=0}^{n-i} a_{i k}^{j} t^{j} c_{i}^{0 k-n+i}=\sum_{j=0}^{n-i} a_{i k}^{j} c_{i}^{j k-n+i}, \quad(k \geqq n-i) .
\end{aligned}
$$

Here $a_{i k}^{j}$ is the coefficient of $t^{j}$ of the polynomial

$$
F_{n-1 k-1}^{+}\left(s_{i}\right) \cdots F_{n-k 0}^{+}\left(s_{i}\right) F_{n-k-10}^{-}\left(s_{i}\right) \cdots F_{i 0}^{-}\left(s_{i}\right)
$$

if $k<n-i$, or

$$
F_{n-1 k-1}^{+}\left(s_{i}\right) \cdots F_{i k-n+i}^{+}\left(s_{i}\right)
$$

if $k \geqq n-i$.

The support of $T_{i}^{j}(x)$ is contained in $\overline{S_{i}^{j}}$, and the associated numbers on $\boldsymbol{\Lambda}_{i}^{j k}(k=0,1, \ldots, n)$ are all 1 . The associated numbers on $\boldsymbol{\Lambda}_{i}^{j k}\left(j^{\prime} \neq j\right)$ are all zero, i. e., there are no singular spectrums. Therefore, the principal symbol on $\boldsymbol{\Lambda}_{n}^{\text {ok }}$ is given by

$$
\sigma_{\Lambda_{n}^{0 k}}\left(T_{i}^{j}(x)\right)=a_{i k}^{j}\left|P_{\Lambda_{n}^{0 k}}\right| s \sqrt{\left|\omega_{\Lambda_{n}^{0 k}}\right|}
$$

with $k=0,1, \ldots, n$.

Thus, as in the same way of Theorem 3. 8, we have the formula of the Fourier transforms,

$$
\begin{aligned}
& \left.\int T_{i}^{j}(x) \exp (-2 \pi \sqrt{-1}\langle x, y\rangle) d x\right|_{V_{R}^{*}-s_{R}^{*}} \\
& =(2 \pi)^{-n^{\prime} / 2-n s_{i}} 2^{-n(n-1) v / 4} \sum_{k=0}^{n} a_{i k}^{j}|\operatorname{det} y|_{k}^{-s_{i}-\frac{n+1}{2}},
\end{aligned}
$$

where $n^{\prime}, v$ are defined in (3.46) and $s_{i}$ is defined in (2.50).

By computing $a_{i k}^{j}$ explicitely and by putting $b_{i k}^{(n) j}=(2 \pi)^{-\frac{n^{\prime}}{2}-n s_{i}} 2^{-\frac{n(n-1) v}{4}}$. $a_{i k}^{j}$, we have the following formula of the Fourier transforms of $T_{i}^{j}(x)$.

Theorem 4.3. 1. In the case of $\boldsymbol{V}=\boldsymbol{S} \boldsymbol{y} \boldsymbol{m}(n, \boldsymbol{R})$.

$$
\begin{aligned}
& \left.\int T_{i}^{j}(x) \exp (-2 \pi \sqrt{-1}\langle x, y\rangle) d x\right|_{V_{\boldsymbol{R}}^{*}-S_{\boldsymbol{R}}^{*}} \\
& =\sum_{k=0}^{n} b_{i k}^{(n)} j|\operatorname{det} y|_{k}^{-(n-i) / 2}
\end{aligned}
$$

where

$$
\text { 1) } \begin{aligned}
n-i & \equiv 0, j \equiv 0 \quad(\bmod .2) \\
b_{i k}^{(n) j} & =(2 \pi)^{(n+1)(2 i-n) / 4} 2^{-n(n-1) / 4} \prod_{p=1}^{n-i} \Gamma(p / 2) \\
& \times \exp \left(\frac{\pi}{4} \sqrt{-1}((n-i)(n-2 k+2 i)+2 i j)\right)\left(\begin{array}{c}
(n-i) / 2 \\
j / 2
\end{array}\right)
\end{aligned}
$$


2) $n-i \equiv 0, j \equiv 1(\bmod .2)$ $b_{i k}^{(n) j}=0$

3) $n-i \equiv 1, j \equiv 1(\bmod .2)$

$$
\begin{aligned}
& b_{i k}^{(n) j}=(2 \pi)^{(n+1)(2 i-n) / 4} 2^{-n(n-1) / 4} \prod_{p=1}^{n-i} \Gamma(p / 2) \\
& \quad \times \exp \left(\frac{\pi}{4} \sqrt{-1}((n-i)(n-2 k+2 i)+2 i(j+1))\right)\left(\begin{array}{l}
(n-i-2) / 2 \\
j(-1) / 2
\end{array}\right)
\end{aligned}
$$

4) $n-i \equiv 1, j \equiv 0(\bmod .2)$

$$
\begin{aligned}
& b_{i k}^{(n) j}=(2 \pi)^{(n+1)(2 i-n) / 4} 2^{-n(n-1) / 4} \prod_{p=1}^{n-i} \Gamma(p / 2) \\
& \quad \times \exp \left(\frac{\pi}{4} \sqrt{-1}((n-i)(n-2 k+2 i)+2 i(j+1)+4 k-2)\right)\left(\begin{array}{c}
(n-i-2) / 2 \\
j / 2
\end{array}\right)
\end{aligned}
$$

2. In the case of $\mathbb{V}=\operatorname{Her}(n, \mathbb{C})$.

(4. 52) $\left.\int T_{i}^{j}(x) \exp (-2 \pi \sqrt{-1}\langle x, y\rangle) d x\right|_{V^{*}-S^{*}}=\sum_{k=0}^{n} b_{i k}^{(n) j}|\operatorname{det} y|_{k}^{i-n}$

where

$$
\begin{aligned}
b_{i k}^{(n) j} & =(2 \pi)^{-(n(n-2 i)+(n-i)) / 2} 2^{-n(n-1) / 2} \prod_{p=1}^{n-i} \Gamma(p) \\
& \times \exp \left(\frac{\pi}{2} \sqrt{-1}((n-i)(2 k-n)+2(i+1) j)\right)\left(\begin{array}{c}
n-i \\
j
\end{array}\right)
\end{aligned}
$$

3. In the case of $\mathbb{V}=\mathbb{H e r}(n, \mathbb{H})$.

(4. 54) $\left.\int T_{i}^{j}(x) \exp (-2 \pi \sqrt{-1}\langle x, y\rangle) d x\right|_{V^{*}-S^{*}}=\sum_{k=0}^{n} b_{i k}^{(n) j}|\operatorname{det} y|_{k}^{2(i-n)}$

where

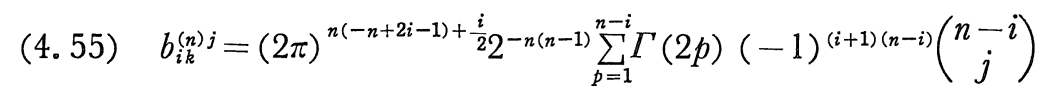

Lastly, we shall give a relation formula between the $\mathbb{G}^{(n) 1}$-invariant measure $d \nu_{i}^{j}$ on $S_{i}^{j}$ and the invariant measure $d g^{(n) 1}$ on $G^{(n) 1}$. From now on, we denote by $G^{(n)}$ or $G^{(n) 1}$ instead of $G_{R}$ or $G_{R}^{1}$, respectively and $\boldsymbol{V}_{\boldsymbol{R}}$ is denoted by $\mathbb{V}^{(n)}$.

The $G^{(n) 1}$-orbit $S_{i}^{j}$ is a homogeneous space. The integration on $G^{(n) 1}$ by a $G^{(n) 1}$-invariant measure $d g^{(n) 1}$ is divided into the integration on $S_{i}^{j}$ by the $G^{(n) 1}$ invariant measure and the integration on the fiber $G_{x}^{(n) 1}\left(x \in S_{i}^{j}\right)$ by the left invariant measure. We shall determine the measure on $G_{x}^{(n) 1}$ naturally defined by determining the measure 
on $\boldsymbol{S}_{i}^{j}$.

Let $x_{0}^{(j)}=I_{n}^{(j)}$. Then $x_{0}$ is a generic point of the $G^{(n)}$-orbit $V_{j}^{(n)}$. We can identify $V_{j}^{(n)}$ and $G^{(n)} / G_{x_{0}^{(j)}}^{(n)}$ where $G_{x_{0}^{(j)}}^{(n)}$ is the isotropy subgroup of $G^{(n)}$ at $x_{0}^{(j)}$. There exists the $G^{(n)}$-invariant measure $|P(x)|^{-n^{\prime \prime n}} d x^{(n)}$ on $\boldsymbol{V}-\boldsymbol{S}$. Here $n^{\prime}=\operatorname{dim} \boldsymbol{V}^{(n)}$ and $d x^{(n)}$ is the Euclidean volume element on $V^{(n)}$ which is defined in (3.6). Then we have

$$
\begin{aligned}
& \left.|P(x)|^{-n^{\prime} / n} d x^{(n)}\right|_{x=x_{0}(j)}=\left|\bigwedge_{i \leqq j} d X_{i j}\right| \\
& \text { (resp. }=\left|\left(\bigwedge_{i=1}^{n} d X_{i i}\right) \wedge\left(\bigwedge_{i<j}\left(d X_{i j}^{1} \wedge d X_{i j}^{2}\right)\right)\right|, \\
& \left.\quad=\left|\left(\bigwedge_{i=1}^{n} d X_{i i}\right) \wedge\left(\bigwedge_{i<j}\left(d X_{i j}^{1} \wedge d X_{i j}^{2} \wedge d X_{i j}^{3} \wedge d X_{i j}^{4}\right)\right)\right|\right),
\end{aligned}
$$

with

$$
\begin{aligned}
& x=\left(X_{i j}\right) \in \boldsymbol{S y m}(n, \boldsymbol{R})=\mathbb{T}_{x_{0}^{(j)}} \boldsymbol{V}^{(n)} \\
& \text { (resp. } x=\left(X_{i j}\right) \in \operatorname{Her}(n, \boldsymbol{C})=\boldsymbol{T}_{x_{0}^{(j)}} \boldsymbol{V}^{(n)} \\
& \text { and } \left.X_{i j}=X_{i j}+\sqrt{-1} X_{i j}(i<j)\right) \text {, } \\
& x=\left(X_{i j}\right) \in \operatorname{Her}(n, \boldsymbol{H})=\mathbb{T}_{x_{0}^{(j)}} \mathbb{V}^{(n)} \\
& \text { and } \left.X_{i j}=X_{i j}^{1}+e_{1} X_{i j}^{2}+e_{2} X_{i j}^{3}+e_{1} e_{2} X_{i j}^{4} \quad(i<j)\right) \text {. }
\end{aligned}
$$

Thus, there exists a left invariant measure $d g_{x_{0}^{(j)}}^{(n)}$ on the isotropy subgroup $G_{x_{0}^{(j)}}^{(n)}$ satisfying

$$
\begin{aligned}
& \int f(g) d g^{(n)}=\int\left(\int f(x \cdot h) d g_{x_{0}^{(j)}}^{(n)}(h)\right)|P(x)|^{-n^{\prime} / n} d x^{(n)}, \\
& \left(f \in C_{0}^{\infty}(\boldsymbol{G})\right)
\end{aligned}
$$

where $d g^{(n)}$ is a $G^{(n)}$-invariant measure on $G^{(n)}$ and $x \in G^{(n)} / G_{x_{0}^{(j)}}^{(n)} \cong V_{j}^{(n)}$. Let $\mathscr{G}^{(n)}$ be the Lie algebra of $G^{(n)}$, which is identified with the tangent space of $G^{(n)}$ at the neutral element $e \in G^{(n)}$. We normalize the $G^{(n)}$-invariant measure $d g^{(n)}$ on $G^{(n)}$ by

$$
\begin{aligned}
\text { (4.58) } \quad d g^{(n)}(e)=\left|\bigwedge_{1 \leqq i, j \leqq n} d A_{i j}\right| \text { with }\left(A_{i j}\right) \in \mathscr{G}^{(n)}=\mathbb{M}(n, \boldsymbol{R}) \\
\text { (resp. } d g^{(n)}(e)=\left|\bigwedge_{1 \leqq i, j \leqq n}\left(d A_{i j}^{1} \wedge d A_{i j}^{2}\right)\right| \\
\text { with }\left(A_{i j}^{1}+\sqrt{-1} A_{i j}^{2}\right) \in \mathscr{G}^{(n)}=\mathbb{M}(n, \boldsymbol{C}), \\
d g^{(n)}(e)=\left|\bigwedge_{\substack{1 \leqq i, j \leqq n \\
\text { with }}}\left(d A_{i j}^{1} \wedge d A_{i j}^{2} \wedge d A_{i j}^{3} \wedge d A_{i j}^{4}\right)\right| \\
\end{aligned}
$$


The vector space $\mathscr{G}^{(n)} / \mathscr{G}_{x_{0}}^{(n)}$ is identified with $\mathbb{T}_{x_{0}} \mathbb{V}^{(n)}$ for $x_{0} \in \mathbb{V}-\mathbb{S}$. Then $d x^{(n)}$ is regarded as the volume form on $\mathscr{G}^{(n)} / \mathscr{G}_{x_{0}}^{(n)}$, and hence we have,

$$
d g^{(n)}(e)=\left|d x^{(n)}\left(x_{0}\right) \wedge d g_{x_{0}}^{(n)}(e)\right|
$$

by normalizing $d g_{x_{0}}^{(n)}$ suitably. Especially, for $x_{0}=I_{n}^{(j)}$, the isotropy subgroup is $\boldsymbol{O}(j, n-j, \mathbb{R})$ (resp. $\mathbb{U}(j, n-j, \mathbb{C}), \mathbb{U}(j, n-j, \mathbb{H}))$. The Lie algebra $\mathscr{G}_{x_{0}}^{(n)}$ is written as

$$
\begin{aligned}
\mathscr{G}_{x_{0}}^{(n)} & =\left\{A \in \mathbb{M}(n, \mathbb{R}) ; A x_{0}+x_{0}^{t} A=0\right\} . \\
\left(\text { resp. } \mathscr{G}_{x_{0}}^{(n)}\right. & =\left\{A \in \mathbb{M}(n, \boldsymbol{C}) ; A x_{0}+x_{0}^{t} \bar{A}=0\right\}, \\
\mathscr{G}_{x_{0}}^{(n)} & \left.=\left\{A \in \mathbb{H}(n, \mathbb{H}) ; A x_{0}+x_{0}^{t} \bar{A}=0\right\} .\right)
\end{aligned}
$$

We define the volume form $d g_{x_{0}}^{(n)}(e)$ on $\mathscr{G}_{x_{0}}^{(n)}$ by the relation (4.59) and denote it by $d A_{x_{0}}^{(n)}$, i. e., $d g^{(n)}(e)=\left|d x^{(n)} \wedge d A_{x_{0}}^{(n)}\right|$ with $A \in \mathscr{G}_{x_{0}}^{(n)}$.

We put $x_{i}^{(j)}=\left[\begin{array}{ll}I_{n-i}^{(j)} & \\ & 0_{i}\end{array}\right]$. The tangent space of $\mathbb{S}_{i}^{j}$ at $x_{i}^{(j)}$ is written as

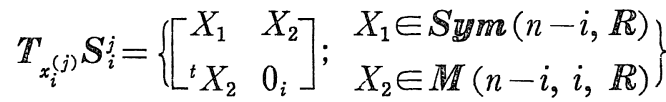

$$
\begin{aligned}
& \text { (resp. } \mathbb{T}_{x_{i}^{(j)}} \mathfrak{S}_{i}^{j}=\left\{\left[\begin{array}{cc}
X_{1} & X_{2} \\
t \bar{X}_{2} & 0_{i}
\end{array}\right] ; \begin{array}{l}
X_{1} \in \mathbb{H e r}(n-i, \mathbb{C}) \\
X_{2} \in \mathbb{M}(n-i, i, \mathbb{C})
\end{array}\right\} . \\
& \left.\mathbb{T}_{x_{i}^{(j)}} \boldsymbol{S}_{i}^{j}=\left\{\left[\begin{array}{cc}
X_{1} & X_{2} \\
t \bar{X}_{2} & 0_{i}
\end{array}\right] ; \quad \begin{array}{l}
X_{1} \in \mathbb{H} \operatorname{Her}(n-i, \mathbb{H}) \\
X_{2} \in \mathbb{H}(n-i, i, \mathbb{H})
\end{array}\right\}\right) 。
\end{aligned}
$$

We define the volume form $\left|d X_{1} \wedge d X_{2}\right|$ on $\mathbb{T}_{x_{i}^{(j)}} \oiint_{i}^{j}$ by

(4.62) $d X_{1}=\bigwedge_{j \leq k} d X_{j k}$ with $X_{1}=\left(X_{j k}\right) \in \mathbb{S} y m(n-i, \mathbb{R})$.

(resp. $d X_{1}=\left(\bigwedge_{j=1}^{n-i} d X_{j j}\right) \wedge\left(\bigwedge_{j<k}\left(d X_{j k}^{1} \wedge d X_{j k}^{2}\right)\right)$,

with $\left(X_{j k}\right) \in \operatorname{Her}(n-i, \mathbb{C})$ and $X_{j k}=X_{j k}^{1}+\sqrt{-1} X_{j k}^{2}$,

$$
d X_{1}=\left(\bigwedge_{j=1}^{n-i} d X_{j j}\right) \wedge\left(\bigwedge_{j<k}\left(d X_{j k}^{1} \wedge d X_{j k}^{2} \wedge d X_{j k}^{3} \wedge d X_{j k}^{4}\right)\right)
$$

with $\left(X_{j k}\right) \in \operatorname{Her}(n-i, \mathbb{H})$ and $\left.X_{j k}=X_{j k}^{1}+e_{1} X_{j k}^{2}+e_{2} X_{j k}^{3}+e_{1} e_{2} X_{j k}^{4}\right)$

and 
(4.63) $d X_{2}=\bigwedge d X_{j k}$ with $X_{2}=\left(X_{j k}\right) \in \boldsymbol{M}(n-i, i, \boldsymbol{R})$.

(resp. $d X_{2}=\wedge\left(d X_{j k}^{1} \wedge d X_{j k}^{2}\right)$

$$
\begin{aligned}
& \text { with }\left(X_{j k}+\sqrt{-1} X_{j k}\right) \in \mathbb{M}(n-i, i, \boldsymbol{C}), \\
d X_{2}= & \wedge\left(d X_{j k}^{1} \wedge d X_{j k}^{2} \wedge d X_{j k}^{3} \wedge d X_{j k}^{4}\right) \\
& \text { with } \left.\left(X_{j k}^{1}+e_{1} X_{j k}^{2}+e_{2} X_{j k}^{3}+e_{1} e_{2} X_{j k}^{4}\right) \in \mathbb{M}(n-i, i, \boldsymbol{H}) .\right) .
\end{aligned}
$$

Then, for the $\boldsymbol{G}^{(n) 1}$-invariant measure $d \nu_{i}^{j}$ on $\boldsymbol{S}_{i}^{j}$ defined in Theorem 4. 2, we have

\section{Lemma 4. 4.}

$$
\begin{aligned}
d \nu_{i}^{j}\left(x_{i}^{(j)}\right) & =(2 \pi)^{\frac{i(i+1)}{4}}\left|d X_{1} \wedge d X_{2}\right| \\
\left(\text { resp. } d \nu_{i}^{j}\left(x_{i}^{(j)}\right)\right. & =(2 \pi)^{\frac{i^{2}}{2}}\left|d X_{1} \wedge d X_{2}\right| \\
d \nu_{i}^{j}\left(x_{i}^{(j)}\right) & \left.=(2 \pi)^{\frac{i(2 i-1)}{2}}\left|d X_{1} \wedge d X_{2}\right|\right)
\end{aligned}
$$

Proof. We shall prove this lemma only in the case of $\boldsymbol{V}=$ $\boldsymbol{S y} \boldsymbol{m}(n, \boldsymbol{R})$. Similar proofs are possible in the cases of $\boldsymbol{V}=\boldsymbol{H e r}(n, \boldsymbol{C})$ and $\boldsymbol{V}=\boldsymbol{H e r}(n, \boldsymbol{H})$.

We denote $\left[\begin{array}{c|c}\check{x}^{n-i} & x_{2}{ }^{i} \downarrow \\ \hline{ }^{t} x_{2} & x_{3}\end{array}\right] \in V^{(n)}$.

We put $\boldsymbol{S}_{i}^{j \perp}=\left\{\left[\begin{array}{cc}I_{n-i}^{(j)} & \\ & x_{3}\end{array}\right] ; x_{3} \in \boldsymbol{S y} \boldsymbol{m}(i, \boldsymbol{R})\right\}$.

Then, $\boldsymbol{S}_{i}^{j \perp}$ intersects with $\boldsymbol{S}_{i}^{j}$ at $x_{i}^{(j)}$ transversally, and

$$
\boldsymbol{T}_{x_{i}^{(j)}} \boldsymbol{S}_{i}^{j \perp}=\left\{\left[\begin{array}{ll}
0_{n-i} & \\
& X_{3}
\end{array}\right] ; X_{3} \in \boldsymbol{S y m}(i, \boldsymbol{R})\right\} .
$$

We take a coordinate system $u=\left(u_{k m}\right)_{1 \leqq k \leqq m \leqq i}$ which satisfies

$$
\left.u_{k m}\right|_{S_{i}^{j \perp}}=\left.x_{n-i+k n-i+m}\right|_{S_{i}^{j \perp}}
$$

and

$$
\boldsymbol{S}_{i}^{j}=\left\{u_{k m}=0 \text { for all } 1 \leqq k \leqq m \leqq i\right\},
$$

in a neighborhood of $x_{i}^{(j)}$. Then

$$
d u_{k m}\left(x_{i}^{(j)}\right)=d X_{3}^{k m},
$$

where $X_{3}=\left(X_{3}^{k m}\right)$ 。 
Let $d \mu$ be the $\mathbb{G}^{(n) 1}$-invariant measure on $\mathbb{S}_{i}^{j}$ normalizing by $d \mu\left(x_{i}^{(j)}\right)=\left|d X_{1} \wedge d X_{2}\right|$. When we regard $d \mu$ as a measure on $\mathbb{V}^{(n)}$, we can write it as

$$
d \mu=f(x) \prod_{k \leqq m} \delta\left(u_{k m}\right)\left|d u \wedge d x_{1} \wedge d x_{2}\right|,
$$

by using a local coordinate system $\left(u, x_{1}, x_{2}\right)$ in a neighborhood of $x_{i}^{(j)}$, where $f(x)$ is an analytic function with $f\left(x_{i}^{(j)}\right)=1$, and $d u=\bigwedge d u_{k m}$, $d x_{1}=\bigwedge_{1 \leq k \leq m \leq i} d x_{k m}$ and $d x_{2}=\bigwedge_{\substack{1 \leq k \leq n-i \\ n-i+1 \leq m \leq n}} d x_{k m}$. Moreover, by regarding (4.66) as a hyperfunction on $\mathbb{V}^{(n)}$, the principal symbol is by definition

$$
(2 \pi)^{-\frac{i(i+1)}{4}} f(x) \sqrt{\left|d u^{*}\right|} / \sqrt{|d u|},
$$

where $\left(u_{k m}^{*}\right)$ is the dual coordinate of $\left(u_{k m}\right)$ and $d u^{*}=\bigwedge_{k \leq m} d u_{k m}^{*}$ Therefore, the principal symbol at $x$ is

$$
\begin{aligned}
& (2 \pi)^{-\frac{i(i+1)}{4}} \sqrt{\left|d u^{*}\right|} /\left.\sqrt{|d u|}\right|_{x=x_{i}^{(j)}} \\
& =(2 \pi)^{-\frac{i(i+1)}{4}} \sqrt{\left|d X_{3}^{*}\right|} / \sqrt{\left|d X_{3}\right|},
\end{aligned}
$$

where $\left|d X_{3}^{*}\right|$ is the volume form $\left.\right|_{k \leqq m} d\left(X_{3}^{*}\right)_{k m} \mid$ on the cotangent vector space $T_{x_{i}^{(j)}}^{*} S_{i}^{j}$ by the dual coordinate $X_{3}^{*}=\left(\left(X_{3}^{*}\right)_{k m}\right)$ of $X_{3}$. On the other hand, the measure $d \nu_{i}^{j}$ on $\boldsymbol{S}_{i}^{j}$ is also a $\mathbb{G}^{(n) 1}$-invariant measure. The principal symbol of the measure $d \nu_{i}^{j}$, regarding as a hyperfunction on $\mathbb{V}^{(n)}$ defined near $x_{i}^{(j)}$, is

$$
\left|P_{\Lambda}\right| S^{i} \sqrt{\left|\omega_{\Lambda}\right|} / \sqrt{\left|d x^{(n)}\right|}
$$

from (4.1). Here $\Lambda$ is the conormal bundle of $\mathbb{S}_{i}^{j}$. In order to write $(4.69)$ by using the coordinate system $\left(u_{k m}\right)$, we restrict $(4.69)$ to $S_{i}^{j \perp}$. We have $\left.P(x)\right|_{s_{i}^{j \perp}}=\operatorname{det}\left(u_{k m}\right)$. Therefore we have

$$
\begin{aligned}
\mathbb{W} \cap \mathbb{T}^{*} \mathbb{S}_{i}^{j}= & \text { The Zariski closure of } \\
& \left\{\left(u, \sigma \operatorname{grad}_{u} \log (\operatorname{det}(u)) \in T^{*} \mathbb{S}_{i}^{j \perp} ; \operatorname{det} u \neq 0, \sigma \in \mathbb{R}\right\} .\right.
\end{aligned}
$$

Let $\pi$ be the projection map from $\mathbb{W}^{\prime} \cap \mathbb{T}^{*} \mathbb{S}_{i}^{j \perp}$ to $\mathbb{S}_{i}^{j \perp}$. Then,

$$
\begin{aligned}
& P_{\Lambda}=P \circ \pi /\left.\sigma^{i}\right|_{\Lambda}, \\
& \omega_{\Lambda}=\frac{\pi^{*}(d u) \wedge d x_{1} \wedge d x_{2} \wedge d \sigma}{\sigma^{i}} /\left.d \sigma\right|_{\Lambda}
\end{aligned}
$$

in $\mathbb{T}^{*} S_{i}^{j \perp}$ where $i^{\prime}=\operatorname{dim} V^{(i)}=\frac{i(i+1)}{2}$. 
By a similar calculations in (3.24), we have

$$
\left|P_{\Lambda}\right|^{s_{i}} \sqrt{\left|\omega_{\Lambda}\right|} /\left.\sqrt{|d x|}\right|_{T^{*} s_{i}^{j \perp}}=c_{0}^{s_{i}} c_{1}^{1 / 2} \sqrt{\left|d u^{*}\right|} / \sqrt{|d u|},
$$

with

$$
c_{0}=\left|(\operatorname{det} u) \cdot \operatorname{det}\left(\operatorname{grad}_{u} \log (\operatorname{det} u)\right)\right|=1,
$$

and

$$
c_{1}=\mid(\operatorname{det} u)^{2 i^{\prime} / i} \cdot\left(\operatorname{Hess}_{u} \log (\operatorname{det} u) \mid=1,\right.
$$

and hence

$$
\left|P_{\Lambda}\right|^{s_{i}} \sqrt{\left|\omega_{\Lambda}\right|} /\left.\sqrt{|d x|}\right|_{x=x_{i}^{(j)}}=\sqrt{\left|d X_{3}^{*}\right|} / \sqrt{\left|d X_{3}\right|} .
$$

Thus we have

$$
\begin{aligned}
d \nu_{i}^{j}\left(x_{i}^{(j)}\right) & =(2 \pi)^{\frac{i(i+1)}{4}} d \mu\left(x_{i}^{(j)}\right) \\
& =(2 \pi)^{\frac{i(i+1)}{4}}\left|d X_{1} \wedge d X_{2}\right| .
\end{aligned}
$$

q. e. d.

We can identify $\boldsymbol{S}_{i}^{j}$ and $\boldsymbol{G}^{(n) 1} / \boldsymbol{G}_{x_{i}^{(j)}}^{(n) 1}$. Let $\mathscr{G}^{(n) 1}$ be the Lie algebra of $G^{(n) 1}$. We can write

$$
\mathscr{G}^{(n) 1}=\left\{A \in \mathscr{G}^{(n)} ; \operatorname{Re}(\operatorname{Tr}(A))=0\right\},
$$

and $\mathscr{G}^{(n) 1}$ is regarded as the tangent space of $G^{(n) 1}$ at the neutral element $e$. We denote the invariant measure on $G^{(n) 1}$ by $d g^{(n) 1}$ and suppose that it is normalized by

$$
\begin{aligned}
& d g^{(n) 1}(e)=\left|\bigwedge_{(i, j) \neq(n, n)} d A_{i j}\right|, \\
& \text { (resp. } d g^{(n) 1}(e)=\mid \bigwedge_{(i, j) \neq(n, n)}\left(d A_{i j}^{1} \wedge d A_{i j}^{2} \wedge d A_{n n}^{2} \mid,\right. \\
& d g^{(n) 1}(e)=\mid \bigwedge_{(i, j) \neq(n, n)}^{1}\left(d A_{i j}^{1} \wedge d A_{i j}^{2} \wedge d A_{i j}^{3} \wedge d A_{i j}^{4}\right) \\
&\left.\wedge\left(d A_{n n}^{2} \bigwedge d A_{n n}^{3} \wedge d A_{n n}^{4}\right) \mid\right) .
\end{aligned}
$$

Let $\mathscr{G}_{x_{i}^{(j)}}^{(n) 1}$ be the Lie algebra of $G_{x_{i}^{(j)}}^{(n) 1}$. Then $\mathscr{G}^{(n) 1} / \mathscr{G}_{x_{i}^{(j)}}^{(n) 1}$ is identified with the tangent space of $\boldsymbol{S}_{i}^{j}$ at $x_{i}^{(j)}$. The volume form $\left|d X_{1} \wedge d X_{2}\right|$ on $\boldsymbol{T}_{x_{i}^{(j)}} \boldsymbol{S}_{i}^{j}$ is regarded as that on $\mathscr{G}^{(n) 1} / \mathscr{G}_{\substack{(j) 1 \\ x_{i}^{(j)}}}^{(n)}$ Note that $\mathscr{G}^{(n) 1}=\mathscr{G}_{x_{i}^{(j)}}^{(n) 1} \oplus$ $\mathscr{G}^{(n) 1} / \mathscr{G}_{x_{i}^{(j) \cdot}}^{(n) 1}$. There exists a left invariant measure $d g_{x_{i}^{(j)}}^{(n) 1}$ on $G_{x_{i}^{(j)}}^{(n) 1}$ normalized by 


$$
d g^{(n) 1}(e)=\left|\left(d X_{1} \wedge d X_{2}\right) \wedge d g_{x_{i}^{(j)}}^{(n) 1}(e)\right|
$$

We can write the Lie algebra $\mathscr{G}_{x_{i}^{(j)}(n) 1}^{(j)}$ as

$$
\mathscr{G}_{x_{i}^{(j)}(n) 1}^{(j)}=\left\{\left[\begin{array}{ll}
A & B \\
0 & G
\end{array}\right] ; \quad \begin{array}{l}
A \in \mathscr{G}_{x_{0}^{(j)}}^{(n-i)} B \in \mathbb{M}(n-i, i, \mathbb{R}) \\
C \in \mathbb{M}(i, \mathbb{R}), \operatorname{Tr}(A)+\operatorname{Tr}(C)=0
\end{array}\right\}
$$

$$
\begin{aligned}
\text { (resp. } \mathscr{G}_{x_{i}^{(j)}}^{(n) 1} & =\left\{\left[\begin{array}{ll}
A & B \\
0 & C
\end{array}\right] ; \quad \begin{array}{l}
A \in \mathscr{G}_{x_{0}^{(j)}}^{(n-i)} B \in \mathbb{M}\left(n-i, i, C^{(j)}\right. \\
C \in \mathbb{M}(i, C), \operatorname{Re}(\operatorname{Tr}(A)+\operatorname{Tr}(C))=0
\end{array}\right\}, \\
\mathscr{G}_{x_{i}^{(j)}}^{(n) 1} & \left.=\left\{\left[\begin{array}{ll}
A & B \\
0 & C
\end{array}\right] ; \quad \begin{array}{l}
A \in \mathscr{G}_{x_{0}^{(j)}}^{(n-i)} B \in \mathbb{R}(n-i, i, \mathbb{H}) \\
C \in \mathbb{M}(i, \mathbb{H}), \operatorname{Re}(\operatorname{Tr}(A)+\operatorname{Tr}(C))=0
\end{array}\right\}\right)
\end{aligned}
$$

with $x_{0}^{(j)}=I_{n-i}^{(j)}$. Then we have

\section{Lemma 4. 5 .}

$$
d g_{x_{i}^{(j)}}^{(n) 1}(e)=\left|d A_{x_{0}^{(j)}}^{(n-i)} \wedge d B \wedge d G\right| 。
$$

Here, $d A_{x_{0}^{(j)}}^{(n-i)}$ is the volume form $d x^{(n-i)}\left(x_{0}^{(j)}\right)$ defined by (4.59), $d B$ is the volume form defined by $\wedge d B_{i j}$ and $d C$ is the volume form $d g^{(i) 1}$ in (4.75).

Proof. Let $h=\left[\begin{array}{ll}A & B \\ D & G\end{array}\right] \in \mathscr{G}^{(n) 1}$ and put $d D=\wedge d D_{i j}$. Then $d g^{(n) 1}(e)=$ $|d A \wedge d B \wedge d C \wedge d D|$. The action of $h$ to $x_{i}^{(j)}$ is

$$
d \rho(h) x_{i}^{(j)}=\left[\begin{array}{cc}
A I_{n-i}^{(j)}+I_{n-i}^{(j)}{ }^{t} \bar{A} & I_{n-i}^{(j)}{ }^{t} \bar{D} \\
D I_{n-i}^{(j)} & 0_{i}
\end{array}\right]=\left[\begin{array}{cc}
X_{1} & X_{2} \\
t \bar{X}_{2} & 0_{i}
\end{array}\right]
$$

So we have

$$
\text { (4.80) } \quad d A=d X_{1} \wedge d A_{x_{0}^{(j)}}^{(n-i)} \text { and } d D=d X_{2} \text {. }
$$

Therefore we have

$$
\text { (4.81) } \quad d g^{(n) 1}(e)=\left|\left(d X_{1} \wedge d X_{2}\right) \wedge\left(d A_{x_{0}^{(j)}}^{(n-i)} \wedge d B \wedge d G\right)\right|,
$$

and hence we have the result.

q. e. d.

Let $d \nu_{x_{i}^{(j)}}$ be the left invariant measure on $G_{x_{0}}^{(x) 1}$ normalized by

$$
d g^{(n) 1}(e)=\left|d \nu_{i}^{j}\left(x_{i}^{(j)}\right) \wedge d \nu_{x_{i}^{(j)}}(e)\right|
$$

Then we have from (4.64), 


$$
\begin{aligned}
d \nu_{x_{i}^{(j)}} & =(2 \pi)^{\frac{-(i+1)}{4}} d g_{x_{i}^{(j)}}^{(n) 1} \\
\text { (resp. } d \nu_{x_{i}^{(j)}} & =(2 \pi)^{-\frac{i^{2}}{2}} d g_{x_{i}^{(j)}(n) 1} \\
d \nu_{x_{i}^{(j)}} & =(2 \pi)^{-\frac{i(2 i-1)}{2}} d g_{x_{i}^{(j)} \cdot(n) 1}^{(n)} .
\end{aligned}
$$

\section{References}

[1] Igusa, J. -i., On certain representations of semisimple algebraic groups and the arithmetic of the corresponding invariants (I), Invent. Math., 12 (1971), 62-94.

[2] — On the arithmetic of Pffafians, Nagoya Math. J., 47 (1972), 169-198.

[3] Gelfand and Silov, Generalized functions 1, Academic Press.

[4] Sato, M. and Kimura, T., A classification of irreducible prehomogeneous vector spaces and their invariants, Nagoya Math. J., 65 (1977), 1-155.

[5] Sato, M. and Shintani, T., On zeta functions associated with prehomogeneous vector spaces, Ann. of Math., 100 (1974), 13-170.

[6] Sato, M., Kashiwara, M., Kimura, T. and Oshima, T., Microlocal Analysis of prehomogeneous vector spaces, Invent. Math., 62 (1980), 117-179.

[ 7 ] Sato, M., Kawai, T. and Kashiwara, M., Microfunctions and Pseudodifferential equa tions, Lecture note in Mathematics, 287, Springer.

[8] Kashiwara, M. and Miwa, T., Microlocal calculus and Fourier transforms of relative invariants of prehomogeneous vector spaces, Surikaiseki Kenkyusho Kokyuroku 238 (1974), 60-147, in Japanese.

[9] Kashiwara, M., Microlocal calculus of simple microfunctions, in Complex Analysis and Algebraic geometrv, ed. by Baily and Shioda (1977), 369-374.

[10] - M., Micro-local Analysis, Proc. of International Congress of Mathematics Helsinki 1978, 1 (1981), 139-150.

[11] Kashiwara, M. and Oshima, T., Systems of differential equations with regular singularity and their boundary value problems, Ann. of Math., 106 (1977), 145-200.

[12] Suzuki, T., Fourier transforms of relative invariants of prehomogeneous vector spaces, Master Thesis, Nagoya University (1975), in Japanese.

[13] - On zeta functions associated with quadratic forms of variable coefficients, Nagoya Math. J., 73 (1979), 117-147.

[14] Milnor and Husemoller, Symmetric bilinear forms, Springer.

[15] Sato, F., Zeta functions in several variables associated with prehomogeneous vector spaces I, Proc. Japan Acad., 57 (1981), 74-79, II, 126-127, III, 191-193.

[16] Zeta functions in several variables associated with prehomogeneous vector spaces I, Functional equations, Tohoku Math. J., 34, No. 3 (1982), 437-483.

[17] - Ibid II, A convergence criterion, Tohoku Math. J., 35 (1983), 77-99.

[18] — Ibid III, Eisenstein series for indefinite quadratic forms, Ann. of Math., 116 (1982), 177-212.

[19] - On zeta functions of ternary zero forms, J. of Fac. Sci. Univ. of Tokyo., 28, No. 3 (1982), 585-604.

[20] Shintani, T., On Dirichret series whose coefficients are class numbers of integral binary cubic forms, J. of Math. Soc. Japan, 24 (1972), 132-188.

[21] - On zeta functions associated with the vector space of quadratic forms, $J$. Fac. Sci. Univ. of Tokyo, 22 (1975), 25-65. 
[22] Siegel, C. L., Uber die Zetafunktionen indefiniter quadratische Formen, Math。Z., 43 (1938), 682-708.

[23] $\longrightarrow$, The average measure of quadratic forms with given determinant and signature, Ann. of Math., 45 (1944), 667-685.

[24] Kimura, T., The $b$-functions and holonomy diyagrams of irreducible regular prehomogeneous vector spaces, Nagoya Math. J., 85 (1982), 1-80.

[25] - Remarks on some combinatrial construction of relative invariants, Thukuba $J$. of Math, 5 (1981), 101-116.

[26] Theory of prehomogeneous vector spaces, "Sugaku 32-2 (1980), 97-119, in Japanese.

[27] - A classification of Prehomogeneous vector spaces of simple algebraic groups, preprint 1980.

[28] Kimura, T. and Muro, M., On some series of regular irreducible prehomogeneous vector spaces, Proc. Japan Acad., 55 (1979), 384-389.

[29] Muro, M., Some prehomogeneous vector spaces with relative invariants of degree four and the formula of the Fourier transforms, Proc. Japan Acad., 56 (1980), 70-74.

[30] - On prehomogeneous vector spaces related to binary cubic forms I, Memoirs of the Fac. of Sci. Kochi University Series A, 1 (1980), 35-57, II, ibid 2 (1981), 75-99.

[31] Weil, A., Sur certain groupes d'operateurs unitaires, Acta Math., 111 (1964), 143-211.

[32] - Sur la formule de Siegel dans la theorie des groups classiques, Acta Math., 113 (1965), 1-87.

[33] Kashiwara, M., Introduction to the theory of hyperfunctions, Ann. of Math. Studies, 93 (1979), 3-38.

[34] Muro, M., Singular spectrums of hyperfunctions and Fourier transforms of group invariant measures on singular orbits of prehomogeneous vector spaces I, Mem. Fac. Sci. Kochi Univ., 4 (1983), 55-88.

[35] - Ibid II, to appear.

[36] Kashiwara, M, $B$-functions and holonomic systems, rationality of roots of $b$-funcitons, Invent. Math., 38 (1976), 33-53.

[37] Suzuki, T., On the Fourier transformations of complex powers of relative invariants associated with prehomogeneous vector spaces, preprint. 
